\title{
Exploring the Design Space for Human-Food-Technology Interaction: An Approach from the Lens of Eating Experiences
}

TOM GAYLER

School of Computing and Communications, Lancaster University, United Kingdom

CORINA SAS

School of Computing and Communications, Lancaster University, United Kingdom

VAIVA KALNIKAITE்

Dovetailed Ltd., Cambridge, United Kingdom

Embedded in everyday practices, food can be a rich resource for interaction design. This paper focuses on eating experiences to uncover how bodily, sensory and socio-cultural aspects of eating can be better leveraged for the design of user experience. We report a systematic literature review of 109 papers, and interviews with 18 professional chefs, providing new understandings of prior HFI research, as well as how professional chefs creatively design eating experiences. The findings inform a conceptual framework of designing for user experience leveraging eating experiences. These findings also inform implications for HFI design suggesting the value of multisensory flavor experiences, external and internal sensory stimulation and deprivation, aspects of eating for communicating meaning, and designing with contrasting pleasurable and uncomfortable experiences. The paper concludes with six charts as novel generative design tools for HFI experiences focused on sensory, emotional, communicative, performative and temporal experiences.

\section{CCS CONCEPTS • Human-centered computing Interaction design theory, concepts and paradigms}

Additional Keywords and Phrases: Human-Food-Interaction; Food; Eating; Experience; Body; Sensory; Emotional, Performative, Design; Chefs; Design space.

\section{ACM Reference Format:}

First Author's Name, Initials, and Last Name, Second Author's Name, Initials, and Last Name, and Third Author's Name, Initials, and Last Name. 2018. The Title of the Paper: ACM Conference Proceedings Manuscript Submission Template: This is the subtitle of the paper, this document both explains and embodies the submission format for authors using Word. In Woodstock '18: ACM Symposium on Neural Gaze Detection, June 03-05, 2018, Woodstock, NY. ACM, New York, NY, USA, 10 pages. NOTE: This block will be automatically generated when manuscripts are processed after acceptance.

\section{INTRODUCTION}

Food is a ubiquitous material in everyday lives, key for how human bodies sustain themselves and experience pleasure, and deeply entwined with social and cultural practices. The growing interest in Human-Food-Interaction (HFI) in HCI has been framed under two main research directions: 'design-around-food' and 'design-with-food' [57]. Work on design-around-food has explored designing for the social experiences that accompany eating food, with less consideration of what or how food is eaten in these contexts. Within this research area, a body of work has addressed commensality, as the social experiences of eating together, in co-located [49] or remote family settings [197], or even collocated wider communities [64]. Additional research on design-around-food includes tracking systems or emerging speculative design. The latter focuses on food as a provocative material that integrates 
technological concerns with broader environmental, health or social concerns [39]. Tracking systems within design-around-food research have focused on food intake and calory counting apps [138], dieting apps [66], food provenance, food miles and carbon emission [87], or sustainability [109], as well as the tracking of food waste for reducing it [47]. In contrast to the design-around-food, the design-with-food area has been less explored. Most work in this space has focused on the grow, preparation or consumption of food [31]. However, the experience of eating per sei and how it can be better designed for has been limitedly brought to the foreground in HCI research. One reason for this may be the challenges of interacting "with food" beyond the traditional screen-based interfaces [140], for which novel taste- or smell-based interfaces are much needed. Moreover, even in the context of eating or dining, much of previous work on design-with-food has prioritized the socio-cultural aspects [63,79], but less so the eating experiences and their bodily aspects [6]. In addition, while the sensory aspects of eating have been the focus of interdisciplinary research beyond HCI such as in food sciences $[10,173]$ or arts $[34,102,116]$, their HCI exploration has been limited [173]. This is surprising given the growing HCI interest in the human body and its role in interaction design [4].

This paper aims to address this gap, by focusing foremost on the less explored eating experiences. Our working definition of eating experiences is that they represent subjective phenomena integrating sensory, aesthetic, emotional and experiential aspects [75]. Such aspects are also key for characterizing user experience of interacting with technology [73]. The somatic turn in HCI, building on phenomenology and post-Cartesian philosophy [118] has brought forward the body, its sensory experiences, and how they can be leveraged not only for pleasure and engagement but also for reflection and making sense of the world $[4,80,81]$. We argue that food can be a particularly valuable resource for design [198], given its unique relationship to the body [123], being experienced both from outside the body (smell, sound, vision and touch), in the mouth (taste, smell, texture and temperature), and inside the body (digestion and metabolization). As work on food waste practices revealed, as bio-material, food presents additional interesting design affordances of being both edible and degradable, thus, arguably more sustainable than traditional design materials [56].

By focusing on eating experiences we wish to uncover more on the role of the body, and how it can be better leveraged within the design process. Food of course is also essential in the broader context, since eating experiences are often part of rich social and cultural practices, both mundane and celebratory [5]. Thus, our work also considers such socio-cultural aspects, albeit not by themselves, but insofar as they support the eating experiences per sei. We report on a systematic review of 109 papers focused on eating experiences, and an interview study with 18 chefs exploring their creative design for eating experiences. Such triangulation allowed us to both take stock of the HFI research that has prioritized eating experiences, and to unpack chefs' creative design with food to support, arguably, aesthetically rich eating experiences. We aim to integrate understandings from these two studies to frame and better support the design space for user experience in HFI, by addressing the following research questions:

- How have eating experiences been leveraged and designed for in previous HFI research?

- How do professional chefs to design for eating experiences and their aesthetics?

- What is the space of designing for user experience in HFI involving eating experiences?

- How can we better support the understanding and exploration of this design space?

Our work makes three main contributions. First, we uncovered fresh understandings regarding HFI based systems as supporting mostly the sensory and emotional aspects of eating experiences, with less focus on cognitive or social ones, and that common technologies addressed both external and internal senses usually for healthy eating. The interview study highlighted the value of flavor-emotion mapping, as well as innovative uses of taste, 
smell and visual stimuli for both pleasurable and uncomfortable experiences. These findings informed a conceptual framework of designing for user experience, leveraging eating experiences, and positioning the mouth and the gut as new sites for HFI. Second, we articulated novel implications for HFI design research, leveraging sensory and emotional aspects of eating experiences, as well as cultural and performative ones. In particular, we suggest the value of focusing on multisensory flavor experiences, external and internal sensory stimulation and deprivation, flavor-emotion and flavor-emotional memory mapping, leveraging social and cultural aspects of eating to communicate meaning, and designing for dramaturgical climax by contrasting and balancing pleasurable and uncomfortable experiences. Third, we developed six charts as novel HFI inspirational and generative design tools focusing on sensory, emotional, performative, communicative, and temporal aspects of eating experiences.

\section{STUDY 1: SYSTEMATIC LITERATURE REVIEW}

The aim of the systemic literature review was to explore how eating experiences have been leveraged and designed for in previous HFI research with a specific focus on bodily experiences, the purposes they were designed for, and the different technologies supporting such experiences. This study focuses on the first Research Question of how eating experiences have been leveraged, and designed for in previous HFI research.

\subsection{Method}

While previous Human-Food Interaction (HFI) research [13,25] has used categories related to the interaction with food from sourcing and preparing, to waste disposal, our review focuses on papers that mention interactions involving eating experiences per sei [4]. We searched the ACM Digital Library for papers published between January 2007 and September 2019. For full text search we used the roots of three main words relevant to eating experiences: "food", "interact*" and "design" to ensure sufficient breadth of papers, subsequently narrowed down through the following roots of words used for searching the papers' keywords and abstracts: "food*”, "eat*”, "tast*”, "flavo*", "din*", "edibil*" or "tableware".

As illustrated in PRISMA diagram (Fig. 1), this search returned 912 papers including 2 duplicates. At the screening stage, 23 papers we removed since they did not report on new studies, consisting instead of workshop proposals, SIGs or panels. From the remaining 887 papers, after reading their titles and abstracts, we excluded 755 papers mentioning experiences about food albeit without any direct consumption such as grocery shopping applications or diet tracking apps, food journaling or eating disorders where the experiences of the eating food have not been considered. This led to 132 papers that mentioned the experience of eating food or food-like stimuli. The eligibility of these papers was assesses based on their full text, with further 23 paper being excluded: 8 of these papers did not involve eating experiences, and 15 papers were agenda setting without presenting new research such as user studies or system design. This left 109 papers that were included in the analysis, which are preceded by an asterisk in the reference list. The outcomes of each of these steps were carefully discussed by the first and second author to ensure consistent use of the criteria. 


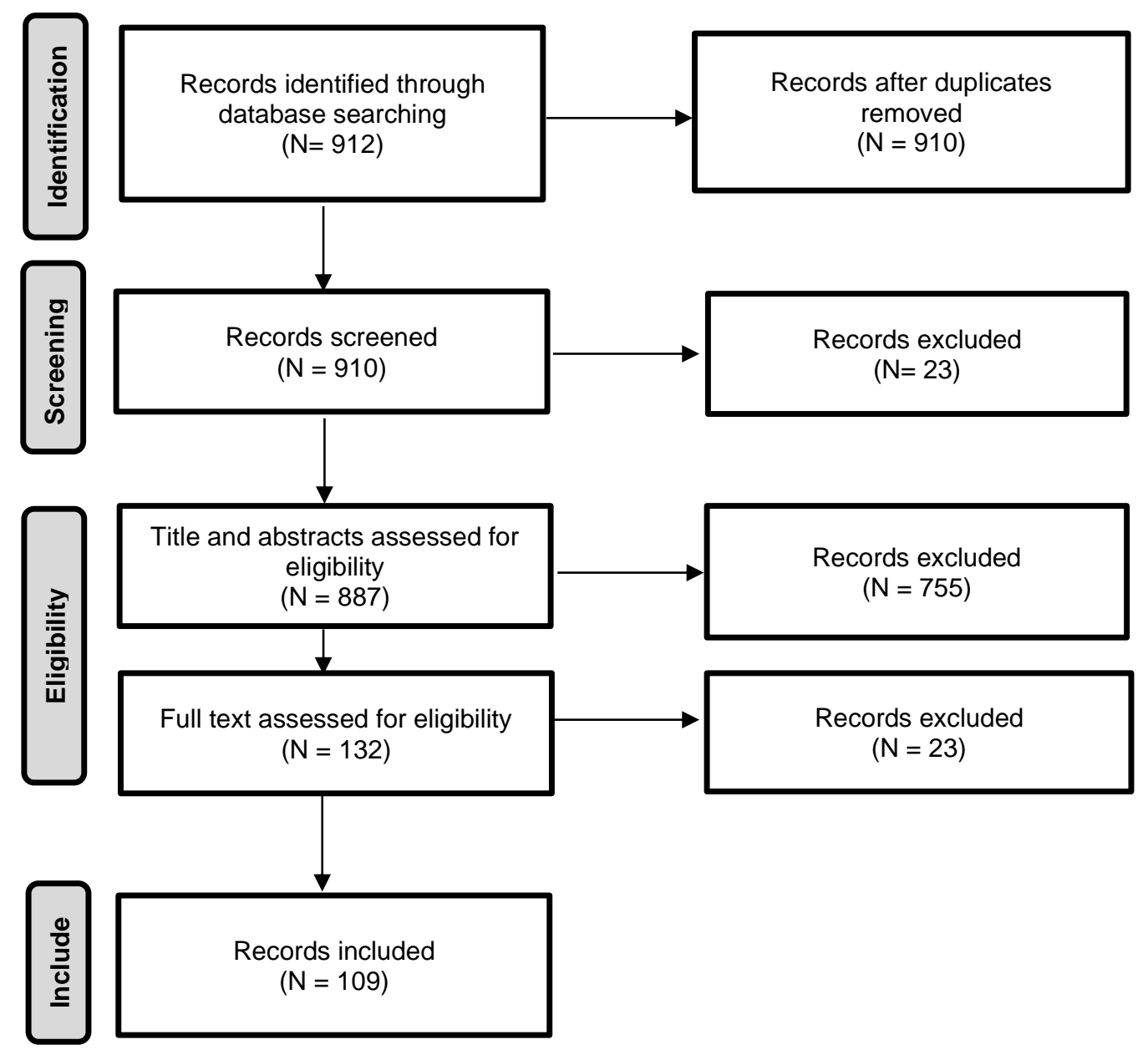

Figure 1 PRISMA flow diagram showing the number of papers at each stage of the systematic review

The coding scheme was iteratively developed through extensive conversations, including three main families of codes that capture the relationship between food and human body ("Body codes"), the purpose of the system or type of eating experience being designed for ("Purpose codes"), and the type of technology supporting these eating experience ("Technology codes"). The rationale for these three main groups of codes relates to the aim of the HFI systematic review, namely, to uncover design knowledge around how the body and technology could be brought together through the experience of eating food. In particular, these three families of codes represent key elements of human-food-interaction, thus the focus on bodily aspects of human users, eating experience of food and its aspects, and on technology mediating the interaction between users and food. The specific codes under each family were identified and iteratively revised especially through independent coding of 10 papers by the first two authors, which led to increased clarity in the definition of a small subset of codes pertaining to the touch and flavor experience, dining systems and technology for moving food and automated dispensing. The final set of codes for each of the three families is shown in Tables 1-3. For the Body codes, we identified two main groups of codes: a larger one reflecting food-based stimulation of external senses, traditionally used in HCI, alongside a smaller group 
indicating food-based stimulation of internal senses reflecting promising novel research directions. The Purpose codes contain four groups describing aspects of experience as well as three application domains. The Technology codes include two broader groups, those related to sensory augmentation, and for each of the application domains.

As we focused on eating experiences it is useful to define two terms that have related but distinct meanings: taste and flavor. Taste is the sensation generated by substances' chemical stimulation of the mouth's taste receptors, with the five basic tastes being bitter, sweet, salty, sour and umami, although other potential tastes have been suggested such as fat [32]. Flavor is a complex multisensory experience of eating food that combines taste alongside smell, texture and other sensory inputs into a singular experience (such as 'chocolatey' or 'burnt') [10].

\subsection{Systematic Review Findings}

In this section we describe the relationship between the body and food and in particular the sensory experiences related to eating, continue with the purposes of interacting with food in HFI, and conclude with the role of technology in leveraging bodily-based eating experiences for each of these purposes. The numbers of papers within specific codes or subgroups are mentioned in brackets. The systematic review outcomes highlight that experiences in human-food interaction are sensory rich (Table 1). However, their focus is not only on external senses of taste, smell, touch, sight and hearing, the first two being less explored in interaction design compared to the latter three, but more importantly, also on internal senses related to digestion and metabolization which have been less explored in HCI. Findings also indicate that human-food interactions support both unimodal and multisensory flavor experiences. Unimodal experiences include those where stimuli trigger single sensory pathways, i.e., taste stimulation through electric current applied to tongue [74] which in turn can be further used to impact other aspects of eating experience, i.e., manipulation of visual qualities of a cookie to increase satiety [131].

\subsubsection{Bodily Experiences of Eating}

\begin{tabular}{|c|c|c|}
\hline Categories & Description & Codes (number of papers) \\
\hline \multirow{7}{*}{$\begin{array}{l}\text { External unimodal senses } \\
\text { from eating experience }\end{array}$} & \multirow{6}{*}{$\begin{array}{l}\text { What unimodal external senses were } \\
\text { stimulated? }\end{array}$} & Taste (26) \\
\hline & & Smell (3) \\
\hline & & Touch - mouth (8) \\
\hline & & Visual (27) \\
\hline & & Audio (3) \\
\hline & & Touch - hand (4) \\
\hline & $\begin{array}{l}\text { What unimodal external senses were } \\
\text { deprived? }\end{array}$ & Deprivation (0) \\
\hline \multirow{3}{*}{$\begin{array}{l}\text { External multisensory } \\
\text { eating } \quad \text { experience } \\
\text { (integration of senses) }\end{array}$} & \multirow{2}{*}{$\begin{array}{l}\text { Did the multisensory experiences of flavor } \\
\text { involve real food and drinks? }\end{array}$} & Flavor - food (13) \\
\hline & & Flavor - drink (5) \\
\hline & $\begin{array}{l}\text { Did the multisensory experiences of flavor } \\
\text { involve simulated food and drinks? }\end{array}$ & $\begin{array}{l}\text { Flavor, Multisensory } \\
\text { interfaces (8) }\end{array}$ \\
\hline $\begin{array}{l}\text { Internal senses of eating } \\
\text { experiences }\end{array}$ & $\begin{array}{l}\text { What internal senses were leveraged in } \\
\text { HFI? }\end{array}$ & $\begin{array}{l}\text { Digestion, metabolization } \\
(12)\end{array}$ \\
\hline
\end{tabular}

Table 1 Categories and codes for bodily experience of eating 
The most common stimuli used to design for unimodal external experiences of food were visual ( $\mathrm{n}=27$ papers), and taste stimuli $(n=26)$. Taste sensation has been less explored in HCI, and our review findings indicate that it has been stimulated through two distinct pathways: food or drinks $(n=16)$, and electronic stimulation $(n=10)$. Interestingly, among the 5 basic tastes (bitter, salty, sour, sweet, and umami), the reviewed work has focused on interactive systems leveraging only a few rather than each of the 5 tastes. For instance, sweet and sour were the most common tastes, explored in 20 and 19 papers, respectively, bitter in 17, salty in 14 and umami in just 7 papers. In contrast, only 6 papers considered experiences with all 5 tastes [55,139,148,180-182], while 4 papers included them all except for umami $[78,147,149,178]$. Moreover, sweet - sour, and sweet - bitter pairings were considered by only $4[20,123,125,185]$, and $3[24,56,118]$ papers, respectively, usually to support contrasting experiences. The remaining 9 papers focusing on taste considered other combinations [121,127,141,146,183] or single tastes $[74,126,128,166]$.

Alongside taste, the other chemical sense is smell $(n=3)$ which has been more commonly used alongside taste and visual stimuli to stimulate multisensory rather than unimodal experiences, like for instance in VR systems showing mediated visualizations of food $[130,132,176]$.

Visual $(n=26)$ and audio $(n=3)$ modalities of interaction are more common in HCI. Systems employing visual modality related to the shape of the food $(n=16)$, or to the subtle alteration of food size or color $(n=11)$ in order to influence perception of satiety or flavor. Auditory systems often leveraged sound stimuli during eating, or their manipulation in order to influence the perception of flavor [104,178,187]. Haptic stimuli related to systems exploring haptic sensations in the mouth $(n=8)$, and the hand $(n=5)$. For instance, mouth based haptic experiences related not only to the perception of textural aspects of the food [111], but also to its exploration through licking [19], or thermal stimulation of the mouth through Peltier modules [147,148,166,174]. Hand-based food-body experiences involved manipulating food forms [67,202], and thermal stimulation of mouth and lips [194,196,197].

In contrast to unimodal experiences, most of the papers on multisensory ones focused on flavor. Such papers involved the simultaneous stimulation of multiple sensory pathways, more often as combined taste and visual stimuli to create for instance lemony flavor from pure water, colored, by using LED lights of yellow color [153], or taste and thermal stimuli to create sweet tasting flavors [166]. Indeed, taste and visual stimuli were used together in 4 systems [150,153-155] and then with smell in a further system [156]. Moreover, 2 systems paired taste with thermal stimuli [151,152] and only 1 integrated taste, thermal and smell stimuli together [157]. Within these multisensory interfaces, taste, visual and thermal stimuli were predominantly digital such as electronic taste, light for manipulating color, and Peltier modules for thermal stimulation. In contrast, smell stimuli were predominantly chemical, i.e., sniffing volatile scents that support orthonasal olfaction which differs from retronasal olfaction stimulated by swallowing food; it is the latter which when combined with gustatory stimuli contribute to flavor [169]. Taste was stimulated in all 8 interactive systems [150-157], visual appearance in 5 [150,153-156], smell in $4[151,152,156,157]$ and touch via thermal stimulation in $3[151,152,157]$.

Both unimodal and multisensory food-based experiences were predominantly triggered through chemical stimuli (food, $n=10$; drink, $n=5$ ), and to a lesser extent through a mix of chemical and electrical stimuli $(n=8)$. When food itself was used as stimuli, it consisted of a wide range of solid and semi-solid foodstuffs such as chocolate [90,111], ice-cream [188], cotton candy [67], carrots with sauce [42] alongside less used non-food materials such as fabrics [19]. When drinks were used as stimuli, they consisted usually of concentrated sweet-flavored drinks, such as energy drinks [69,94-96,178]. For both unimodal sensory and multisensory experiences, interactive systems focused mostly on augmenting them by increasing the intensity of the stimuli and their perception. 
Interestingly, no papers reported sensory deprivation, despite attempts to stimulate sensory experiences in the absence of chemical stimuli (i.e. without consuming food or drinks) like in the case of electronic taste [150-157].

Beside the predominant focus on external senses, fewer papers have explored internal ones pertaining to eating, such as digestion and metabolization and their associated sensations arising from chewing or licking. Internal senses have been explored by 13 papers, with 6 focusing on metabolization of food and how it is impacted by eating . The latter explored the provision of energy drinks in response to physical activity [94-96], experimental approaches that used bodily response to hack diets through soylent-type products $[38,41]$ and the impact of chocolate on cognition and mood [80]. In addition, 3 papers focused on digestion through a sensory informed experimental approach $[38,41]$ to food allergies [88], while the other 2 papers focused on gut as a space for gaming; one used VR to create a visualization of digestion and how it is impacted by chewing [8], while the other used ingestible sensors as input devices for gameplay [17]. Chewing, as a key component of digestion, was the focus of 3 papers; the previously mention VR game that prompted chewing to support digestion [8] and two further gaming experiences, one used chewing as input [7] and one aimed at rehabilitation for those with facial injuries [191]. Not at least, licking, as another component of digestion, was explored by 2 papers: one used the tongue provocatively to explore non-edible environments and materials [19], while the other framed licking as playful [188].

\subsubsection{The Purposes of Human-Food-Interactions in $\mathrm{HCl}$}

Findings identified 13 such purposes, including 9 related to design of eating experience, and 4 related to specific application domains for such experiences. These 9 purposes can be broadly grouped in those focusing on sensory, emotional, cognitive, and social aspects of eating experiences (Table 2).

\begin{tabular}{|c|c|c|}
\hline Categories & Description & Codes (number of papers) \\
\hline \multirow{3}{*}{$\begin{array}{l}\text { Sensory aspects of eating } \\
\text { experiences }\end{array}$} & \multirow{3}{*}{$\begin{array}{l}\text { How were the sensory aspects of } \\
\text { eating leveraged in HFI? }\end{array}$} & Taste stimulation (15) \\
\hline & & $\begin{array}{l}\text { Flavor stimulation through food, i.e., internal } \\
\text { flavor (19) }\end{array}$ \\
\hline & & $\begin{array}{l}\text { Flavor stimulation through food and } \\
\text { environmental stimuli, i.e., external flavor (9) }\end{array}$ \\
\hline \multirow{2}{*}{$\begin{array}{l}\text { Emotional aspects of } \\
\text { eating experiences }\end{array}$} & \multirow{2}{*}{$\begin{array}{l}\text { How were the emotional aspects of } \\
\text { eating leveraged in HFI? }\end{array}$} & Playfulness (28) \\
\hline & & Emotion-taste/flavor relationship (7) \\
\hline \multirow{2}{*}{$\begin{array}{l}\text { Cognitive aspects of eating } \\
\text { experiences }\end{array}$} & \multirow{2}{*}{$\begin{array}{l}\text { How were the cognitive aspects of } \\
\text { eating leveraged in HFI? }\end{array}$} & Storytelling (6) \\
\hline & & Data edibilization (12) \\
\hline $\begin{array}{l}\text { Social aspects of eating } \\
\text { experiences }\end{array}$ & $\begin{array}{l}\text { How were the social aspects of eating } \\
\text { leveraged in HFI? }\end{array}$ & $\begin{array}{l}\text { Commensal (7), } \\
\text { Communicative (3) }\end{array}$ \\
\hline \multirow[t]{3}{*}{$\begin{array}{l}\text { Application domains for } \\
\text { eating experiences }\end{array}$} & \multirow{3}{*}{$\begin{array}{l}\text { What were the application domains } \\
\text { for leveraging the sensory, } \\
\text { emotional, cognitive, or social } \\
\text { aspects of eating in HFI? }\end{array}$} & $\begin{array}{l}\text { Healthy eating: persuasive, } \\
\text { regulation (29) }\end{array}$ \\
\hline & & Dining (14) \\
\hline & & Assistive dining (4) \\
\hline
\end{tabular}

Table 2 Categories and codes for purposes of $\mathrm{HFI}$ 


\subsubsection{Sensory aspects of eating - New taste stimulation}

An important outcome is that many interactive systems have been designed with the explicit purpose of supporting novel taste stimulation, as shown in 15 papers [74,78,118,126,128,135,139,141,146-149,166,178,185]. These systems aim to create or augment such novel taste experiences, to increase pleasure through novel taste or novel food forms and their texture. A large body of work in this space is on electronic taste devices which stimulate taste sensation in the absence of chemical stimuli. Such electronic taste devices could significantly extend the reach of taste-based interactions, beyond the consumption of food per sei. Two thirds of these papers (10/15) use electric taste stimulation which can be broadly grouped in 3 types of devices which all work by using the flow of electric current to stimulate the taste sensation. The first and most common type uses a bespoke tongue probe to apply electric current to the tongue [141,146-149,166]. The second type uses a single pole apparatus, such as a fork or chopstick in which electric current passes through the mouth and body via a second contact point of the hand holding it $[74,126,128]$. The third type uses a device with two opposite polarity probes, for instance a straw, with the tongue used to connect the circuit $[74,128]$.

The systems that use chemical stimuli for taste experience rely on manipulating taste experience of a base food or drink through changing its color through lighting [78,135], or by listening to specific music during eating $[118,178,185]$. Whilst eating experiences are sites for social interaction (see Dining 2.2.3.2) this approach uses the eating itself to support social communication in a new sensory way. The next two sections look at the integrated multisensory experience of flavor, extending the findings on new taste stimulation.

\subsubsection{Sensory aspects of eating - Food aspects impacting on flavor}

This theme relates to the flavor experience of food. Flavors were described by words like 'chocolatey', coffee or meaty and consist of the integration of different sensory inputs (taste, smell, sight, hearing, touch, digestion, metabolization). By augmenting one or more of the external sensory pathways when eating food, the flavor can be changed. Findings show that 20 papers support this aim [20,24,104,111,130,132,135,148,150-157,180,187,197,203], 16 of which aimed solely at producing flavor experiences, while the other 4 created flavor in support of a specific application such as persuasive tech for healthy eating [130]. 9 of these 16 papers described systems using electric taste integrated with other senses, such as sight to manipulate the appearance of drinks' color through LED lights (e.g. [153]), smell through added scents, (e.g. [156]), or sound by manipulating the sound of eating $[24,104,187]$ in order to influence the perception of flavor. For example, the feeling of food 'crunchiness' was augmented by amplifying the sound of chewing [104], or the appearance of food, and thus the flavor and resulting satiety was augmented through VR [130,132], the flavor of food through lighting [20,135], or the texture of food through food printing [197]. Indeed, other approaches to designing flavor experiences came from 3D food printing: novel textures impacting on flavor [111], or digitally designed molds for personalized flavor experiences [203].

\subsubsection{Sensory aspects of eating - Environmental aspects impacting on flavor}

Whilst the previous section looked at flavor experiences informed solely by foodstuff itself, the papers in this section examined how flavor experiences were informed not only by foodstuff, but also by additional external stimuli within the environment in which the food was eaten. Findings indicate 9 papers $[24,118,121,127,137,174,178,194,196]$ exploring how environmental stimuli impact flavor experiences, making 
them more enjoyable, usually in dining contexts. Most of these papers explored sound stimuli to enhance the experience of flavor, through traditional ambient audio or music $[24,118,121,178]$. They explored for example the impact of different music tracks varying in pitch and volume on the perception of chocolate flavor and texture [24]. Two papers reported on predefined audio stimuli created to support the appreciation of flavor qualities of the food $[24,178]$. For example, a Brazilian cocoa praline eaten to the music of a Brazilian composer, or a field recording of the kitchen in which the chocolate was made [24]. Such applications rely on users' exposure to ambient sound whilst eating. There are 2 papers however that grant greater agency to the users as they are able to generate and modulate sounds themselves through the act of drinking [118,121]. Visual stimuli were also used in this context, for instance to change the visual appearance of the food itself through 3D food printing alongside thermal-haptic experiences as part of multisensory interactions for remote connection [194,196]. Here, we have also seen color washes projected on top of 3D printed food to influence the perception of flavor by augmenting the appearance of food's color [137].

\subsubsection{Emotional aspects of eating - Playfulness}

This group consists of 28 papers [7,8,17,19,20,27-29,44,53,67,81,83-85,94-96,114,121,123,125,159,181$183,188,196]$ that focused on the use of food as part of playful eating experiences from structured game-play to more open ended ones. Interestingly, many of them 12/28 took place in dining contexts where the meal rituals traditionally emphasize the civility [46] and aesthetics of eating, rather than its playfulness or sensory aspects [117]. The others 16/28 focused on play while snacking in home or work contexts. These papers provide an interesting lens into how HFI contributes to more informal dining practices. Almost half of the papers focusing on playfulness $(12 / 28)$ explored interactive systems for dining, particularly to persuade healthy eating (9/12), both through game-based and open-ended play for example to encourage children to eat all their food [53].

Almost half of papers (12) reported on structured, rules-based gaming applications [7,8,17,81,83$85,114,123,125,159,181,182]$ using for instance the eating of healthy food to progress in a mobile game [7,84], or playing a video in which sweet or bitter tasting liquids were provided as outputs from the gameplay [123,125]. In contrast, the open-ended games supported more exploratory approaches where users had increased agency, for example to create music while interacting with the foodstuff or drinking device $[121,188]$. One approach that diverged from this, required users to construct their own, rule-based games from food [81].

Playfulness systems usually draw on taste, as shown in 7 papers $[20,121,123,125,181-183]$ where feedback was delivered both through the presence and absence of stimuli [125], as well as the taste of the stimuli $[123,125,182]$. This particularly draws on the relationship between emotions and tastes explored in the following section. Food itself has been also used both as input and output in playful experiences. Food was used as input in a variety of ways; through lollipop as joystick (where physical movement was captured) $[123,125]$, as a capacitive material that becomes interactive when touched or licked $[187,188]$, and as a material to be chewed, the chewing action being captured as input control for the game [7].

Audio-visual stimuli often as video games were used to explore the role of food in game-based playful experiences, both as input and output [7,30,81,123,125,182], ranging from Minesweeper system using a mouthpiece for delivering tastes as feedback in play [182], to bespoke VR/AR [7,8] game environments. Food has been also used in both physical and mixed reality gaming contexts [81]. A key technology for supporting the use of food as output in video games was the delivery of the stimuli, mostly in the form of pumped liquids into a mouthpiece 
$[123,125,182]$. Capacitive sensing and sounds output underpinned three open-ended playful experiences $[43,121,188]$. Another sensory modality emphasized within playfulness systems was audio. Here, 3 papers $[44,121,188]$ used audio outputs from eating experiences, i.e., crunching, not for the purpose of supporting the flavor experience but as a distinct aspect of playful experience. Such audio outputs rely on electrical conductance to cue the interactive experience, and while 2 papers $[44,188]$ used conductance through food material such as jelly [44] and ice-cream [188], the other used conductance via the mouthpiece of a drinking device [121] allowing users to 'play' music while interacting with it.

Another interesting finding is that over a third of the papers (8/28) leveraging gamification principles focused on internal senses of digestion and metabolization. These includes for instance VR-based games aimed to visualize, and increase awareness of slow chewing and digestion through competitive gameplay against another diner $[7,8]$, or ingestible sensors which scored points in gameplay based on gut temperature [17]. Digestion also involved the act of licking, whose ludic aspect has been explored particularly with foodstuff such as ice cream [188] [19]. With regard to metabolization of food, 3 papers focused on the relation between food as fuel, and exercising, as reflected in playful edibilizations of tracked fitness data through an elaborate fountain that mixes sports drinks in response to heartrate [94-96], also used for collaborative play [96].

\subsubsection{Emotional aspects of eating - Emotion - taste relationship}

Emotions and eating experiences are strongly connected and 7 papers explored this relationship $[55,56,80,123,125,139,182]$. Most of these $(6 / 7)$ reported on the emotional valence and specific taste, particularly the association of bitter taste with negative emotions and their value in interaction design $[55,56,123,125,139,182]$. The other paper focused on the emotional experiences associated with flavor, such as those triggered by comfort foods and their positive impact on mood [80]. In addition, the potential of food for regulating emotions has been explored both at individual [80] and dyadic level, i.e., co-regulation of emotions [56].

From the 7 papers focusing on the relationship between taste and emotional valence, 2 papers considered how taste might be better leveraged by designers of interactive systems [55,139]. Furthermore, 2 papers built on this relationship in interactive systems such as those for communication of affective content to users [56], or for supporting mood boosting through food [80]. The other 3 papers explored it within gaming contexts, using for instance tastes such as sweet or bitter to provide positive or negative feedback, respectively, during gameplay $[123,125,182]$. All gameplay devices that we reviewed relied on pumped liquid stimuli, with liquid stimuli used in [139], solid food in [80], and 3D printed stimuli in [56]. For instance, pumped liquids were used in LOLLio system [125] where a lollipop-type device is both input and output device: as a joystick in the mouth and, and as a delivery tool for pumping liquid taste stimuli. While liquid stimuli have been commonly used for taste-based interactions, given their limited texture and therefore increased control over the flavor experiences, 3D printed food have also emerged as an alternative to solid form taste stimuli [56], allowing the exploration of other aspects of flavor besides taste, namely texture or appearance.

\subsubsection{Cognitive aspects of eating - Storytelling}

An interesting finding is the specific focus on storytelling through eating experiences reflected in 6 papers $[3,20,42,61,69,180]$. Most of these papers explored how foodstuff can be used to capture [61], communicate $[20,69,180]$, or capture and communicate [42] both personal $[42,61]$ and collective stories $[20,69,180]$. For example, 
StreetSauce is an interactive system that supports users to create recipes based on ingredients as autobiographical foodstuff matching their personal narratives of homelessness [42]. Other systems emphasized the performative aspect of eating-based storytelling inspired by multi-sensorial theatre combining audio, visual, and edible stimuli [20], multi-sensory film [180] or VR experiences [69]. Half of these papers described users as storytellers, with food allowing a person to narrate a personal story, either to oneself [61] or to another [3,42]. For instance, a remote food printing system supported remote grandparents telling culturally significant stories through food to their grandchildren [3]. In contrast, the remaining 3 papers have taken the approach where the audience $[20,69,180]$ engaged in eating experiences during storytelling. Storytelling could also elicit emotions since "narrative emotions" have been suggested to indicate how meaning and affect become linguistically interlinked [104]. But rather than being triggered merely through sensory stimulation like in the taste/emotion mapping in the section above, they are triggered by the story's content that becomes personally significant to the subject.

\subsubsection{Cognitive aspects of eating - Data edibilization}

An interesting set of 12 papers focused on data edibilization or data communication through food [73,92,94$96,98,99,124,142,143,164,192]$ where food that is eaten is used to explicitly visualize and communicate data. Often such data is tracked data on bodily practice such as users' physical activities, taking the form of messages printed out on edible materials such as chocolate [92]. Data can also be printed in edible inks onto food [164], or laser cut onto the surface of food [73]. The aim of data edibilization is usually to support deeper user engagement, and reflection on the meaning of the data communicated through the eaten food. An illustrative example is Edipulse $[92,98,99]$ producing chocolate to communicate data about user's completed physical activity, rewarding them as the same time, which in turn indicates the potential for multiple layers of meaning within data edibilization.

Several such systems [85,87-91,113] focused on tracked calory data on physical energy expenditure during exercise, which can become abstracted into digital format, reproduced in a physical, edible format, and then consumed by the originator of the data. The act of eating adds a multisensorial aspect, creating a richer, more embodied experience of data that contrasts with its traditional, limited physical form. This creates an interesting loop, particularly in the case of physical activity $[92,94]$ where foodstuff or drinks such as isotonic sports beverages were used both as reward for the completed running session and as fuel for the next one [94-96]. Beside tracked fitness data, the communicated information also included food-centric data such as nutritional information [73], or food unrelated data such as CV information [142,143] or data on gender inequality in tech [164].

The form of food is the most important quality that is manipulated in this group of papers. Foodstuff that can be easily shaped such as chocolate $[92,98,99]$ or doughs $[142,143]$ are prioritized, with melting points that are not much above room temperature or with material characteristics allowing them to flow under small amounts of pressure. As mentioned above, the foodstuff itself is selected for a particular meaning, such as chocolate as reward $[92,98,99,124]$ or isotonic drinks to refuel [94-96]. However, more often, the specific type of foodstuff is not deliberately considered [142,143] apart from being sugary: much preferred foods such as chocolate [92,98,99], fried sweet doughs [142,143], or sweet pies [164] are likely to boost engagement rather than to support specific flavor experience [92,94-96,98,99,124,142,143]. Also related to form is the focus of 11 papers on the precise delivery of food's amount, either though pumped liquids [94-96] or food printing, both 2D $[92,98,99,124,142,143,164]$ and 3D [192]. Also in the context of form, beside whole foodstuff being used to 
communicate data, 11 papers explored food surfaces as spaces for communication [73]. This approach maintains the foodstuff's form but uses laser cutting to inscribe visual information onto the food itself.

\subsubsection{Social aspects of eating: Commensal, cooperative and communicative}

The final set of 14 papers covers the social aspects of eating experiences $[8,20,93,106,119,122,126,136,147,166,194-197]$. These papers covered three main social aspects, which we identified as commensal, cooperative, and communicative. Papers with commensal social aspects $[20,93,106,136,194,196,197]$ shared similarities with pre-exiting HCI work on technologies for commensality $[32,49]$, but they particularly used technology to leverage the eating experiences for social bonding, instead of merely supporting the social contexts of eating. Here we have for instance companion robots for dining with [93], VR systems for eating together with remote strangers as dining elderly partners [106], or video conference-based interactive system supporting time-shifting co-dining so that people can remotely share dinner with their loved ones, whose dining experiences have been previously recorded at an earlier time zone [136]. Papers supporting cooperative eating $[8,119,122,136]$ involved at least two co-diners who have to collaborate so that each one can enjoy a technologically mediated eating experience. Examples include robotic arms for feeding each other [119], VR games controlled by chewing [8], or interactive table which influenced the eating speeds of two diners by tilting, to guide them towards similar speed when the table becomes balanced [122]. Finally, papers supporting communicative social aspects also focused on remotely eating together by supporting co-diners to communicate via messages on 3D printed food or video links [194,196,197]. These papers also supported social communication during eating experiences outside the dining context, for instance through messages in workplace in the form of cookies as 3D printed food [195], whilst other systems explored the link between emotions and taste in order to support more emotional communication during eating experiences $[126,147,166]$.

\subsubsection{Applications Domains}

Apart from the sensorial, emotional, cognitive and social aspects highlighted above, the papers also reflect three applications domains cutting across these aspects such as healthy eating, dining and assisting dining.

\subsubsection{Application Domains - healthy eating: persuasive, tracking and regulation}

When considering desirable 'norms' around food experience, healthier choices represent a growing concern for food experiences in HCI. The 29 papers in this domain focused on shaping experiences that prioritize eating less, usually of the 'wrong' things [8,20,75,100,101,130,131,165,175,176] through both persuasive technologies as well as those for self-tracking and regulation $[17,19,25,41,44,84,86]$, eating more of the 'right' things $[53,68,83-85,158]$, or eating in the 'right' way $[27,28,52,86,114]$ with the latter two groups including mostly persuasive technologies for children. Indeed, almost half of the papers in this domain $(12 / 28)$ focused on younger children, with 6 providing encouragement to eat more healthy food such as vegetables [53,68,83-85,158], and 6 supporting children's attention and good manners during mealtimes [27,28,52,86,114]. A key factor for supporting behavior change is gamification embedded in 5 [8,83-85,114] of the 21 papers on persuasive technologies for healthy eating.

The first group of papers which prioritized eating less food were more broadly aimed at adults and relied on different techniques to persuade users towards desired behavior. Many of these papers described interactive dining tools to persuade behavior change, however they were not game-based but instead attempted to influence 
perception, usually by tricking users into believing that they have eaten more in order to augment satiety and reduce food intake. This is achieved through adaptively weighted cutlery as heavier [75], adaptively sized plates as smaller [165], and 'illusion cups' which augment the apparent volume of liquid in the cup as larger [175]. This perceptual trickery is perhaps most advanced in systems using VR or AR to manipulate the visual appearance of size (larger) or type of foodstuff, for instance by changing a small cookie into one that looks bigger or has different flavors $[130,131,176]$. Interestingly, 3 papers provided direct feedback to help users self-regulate their speed or volume of eating, for instance through the use of colorful lights, or vibration motors within cutlery in order to prompt them towards more conscious choices [20,100,101]. Other 7 papers in this group $[17,19,25,41,44,84,86]$ also supported adults' healthy eating, albeit without the use of gamification for behavior change, but through selftracking and self- regulation. These included apps for self-tracking bodily responses to eating experiences, from gustatory [19] or digestive ones targeting allergy monitoring and management [88], to metabolic ones focused on nutrition and food impact on wellbeing [38,41], for both individual users $[19,88]$ and communities $[38,41]$. Other paper focused on game based on ingestible sensors to support awareness and regulation of gut temperature [17], while another paper focused on self-regulation of craving through a system that automatically dispenses a single chocolate ball every hour allowing user to eat it or return it, thus exercising self-control [90].

The second group consists of papers encouraging eating more of the right foods, which were all aimed at children $[53,68,83-85,158]$, dealing particularly with food dislikes [84] or fussy eaters $[53,68]$. The third group of papers focused again on persuasive systems intended to support good manners during mealtimes and particular dining $[27,28,52,86,114]$. All these papers focused on social aspects of dining and children's behavior that is corrected or reinforced through interactive dining systems [27,28,52,86,114]. For instance, the Kicking Chair is an interactive system dealing with restlessness while seating, as the child can bounce their legs against a rubber band on the chair [27]. Other examples include racing game which rewards focused eating [114], or interactive game to support children's training of fine motor skills by learning to eat with chopsticks as augmented cutlery [29]. Whilst these systems aim to improve behaviors to better support the social experiences of dining, they however have been limitedly explored in such social settings.

\subsubsection{Application Domains - Dining}

This application domain includes 14 papers $[1,2,93,106,119,122,124,136,144,181,183,194,196,197]$ in which the described systems, irrespectively of the sensorial, emotional, cognitive or social aspects they support, were used within the dining contexts: where food is eaten with cutlery and crockery whilst seated. Unsurprisingly, 9 of these systems engaged social aspects of eating experience supporting the purpose of commensality [93,106,119,122,124,136,194,196,197]. 5 of these 9 papers dealt with remote connection $[106,136,194,196,197]$, engaging not only with video links between remote dining partners, as common in much previous research around food [32,49], but also with the eating experience itself. This latter aspect included multisensory interfaces to provide haptic and thermal interactions, as well as food-based messaging via printed words and shapes onto food [194,196,197]. Other approaches to remote dinning captured and altered the speed at which food has been eaten by one partner whose video is used to match the speed at which food is being eaten by the other partner in order to support synchronized eating across different time zones [136]. Other example consists of VR-based spaces in which older adults could eat together, by seeing digital representations of their meals, and of their remote dining partners [106]. Commensality can also be mediated. Indeed, our findings indicate that systems focused on dining 
contexts have also involved robot technologies as anthropomorphic dining partners, intended to augment the social and ludic experience of co-diners. Here we have seen robots as companions that can feed diners [93], or robotic arms that can be used by one dinning partner to co-feed the other $[119,124]$. Interestingly, robots have been used not only to substitute social dining companions like in the former case, but also to increase connection between two dining companions like in the latter. Aside from those related to social dining, 5 out of the 14 remaining papers explored other ways to enrich the eating experience with performative aspects, for instance through magnetic $[1,2,144]$ or acoustic levitation [181,183] for manipulation of food. These systems reported novel technology, designed for performative experiences with food.

\subsubsection{Application Domains - Assistive Dining}

The final application domain consists of systems designed to assist users to eat food $[29,82,105,110,191]$. Three of these papers described systems aimed at users with physical disabilities such as robotic arms which move food to the mouth $[82,105,110]$, intended to provide users with greater autonomy over their eating behavior. The fourth paper described a non-robotic assistive system for the rehabilitation of facial muscles after injury or illness [191]. It consists of a persuasive game controlled through chewing actions: as players chew, they "consume" virtual foods falling down the screen.

\subsubsection{Technologies to Support Eating Experiences in $\mathrm{HCl}$}

We now report on the different types of technologies designed and developed to support eating experiences in the reviewed HFI research (Table 3). These technologies relate to the different identified purposes for human-food interactions (Table 3, col 1). The largest number of technologies (57 papers) support the stimulation of sensory aspects of eating experience especially taste, smell, touch, sight or hearing, with some supporting more than one. Apart from these technologies purposefully supporting merely the sensory aspects of eating experience, the remaining technologies support all aspects of eating experience: sensory, emotional, cognitive and social, albeit differently for each of the three application domains. The second largest group consist of specific types of technologies supporting healthy eating including a range of interactive eating technologies such as smart tableware (20), self-tracking apps (14), games (2), and novel forms of foodstuff delivery (9). The next group of technologies support the dining domain through novel forms of foodstuff, either static (21 papers) or dynamic (3), as well as technologies supporting remote dining (10). The final group include technologies for assistive dining (4).

\begin{tabular}{|l|l|l|}
\hline Categories & Description & Codes (number of papers) \\
\hline Technologies supporting & What types of technologies did & Electronic taste devices (18) \\
\cline { 3 - 3 } $\begin{array}{l}\text { the sensory aspects of } \\
\text { eating experiences }\end{array}$ & $\begin{array}{l}\text { support the stimulation of } \\
\text { sensory aspects of eating } \\
\text { experience? }\end{array}$ & Smell interfaces, i.e., chemical stimuli (4) \\
\cline { 3 - 3 } & & Thermal interfaces, i.e., Peltier elements (7) \\
\cline { 3 - 3 } & & AR/VR systems, i.e., lights and projections (10) \\
\cline { 3 - 3 } & & Visual interfaces (8) \\
\cline { 3 - 3 } & & Audio, ambient interfaces (7) \\
\hline Technologies supporting & What types of technologies did & Smart tableware (20), self-tracking apps (14), \\
the healthy eating domain & support the eating experience & games (2) \\
\hline
\end{tabular}




\begin{tabular}{|c|c|c|}
\hline & $\begin{array}{l}\text { for the domain of healthy } \\
\text { eating? }\end{array}$ & $\begin{array}{l}\text { Novel forms of foodstuff delivery through } \\
\text { automated dispensing technologies (9) }\end{array}$ \\
\hline $\begin{array}{l}\text { Technologies supporting } \\
\text { the dining domain }\end{array}$ & $\begin{array}{l}\text { What types of technologies did } \\
\text { support the eating experience } \\
\text { for the domain of dining? }\end{array}$ & $\begin{array}{l}\text { Static novel foodstuff forms: } 2 \mathrm{D} \text { and } 3 \mathrm{D} \text { printing } \\
(21) \text {, and laser cutting technologies (3) } \\
\text { Dynamic novel foodstuff through acoustic } \\
\text { levitation, magnetic field technologies (3) } \\
\text { Video conferencing, VR integrated with sensory } \\
\text { augmentation, camera tracking, social robots, } \\
\text { novel food forms technologies for remote } \\
\text { dining (10) }\end{array}$ \\
\hline $\begin{array}{l}\text { Technologies supporting } \\
\text { assistive dining domain }\end{array}$ & $\begin{array}{l}\text { What types of technologies did } \\
\text { support eating experience for } \\
\text { the domain of assistive dining? }\end{array}$ & Robotic technologies for (assistive) dining (4) \\
\hline
\end{tabular}

Table 3 Technology categories and codes mapped to the purposes they support

\subsubsection{Augmenting sensory technologies for pleasurable eating experiences}

Technologies that augments sensory experiences of eating food focused predominantly on sensory stimulation of basic tastes [146], although work on supporting other purposes such as communication through taste has also started to emerge [126]. Most technologies for sensory stimulation focused on taste stimulation and in particular electronic taste (18 papers) intended to create taste experiences albeit without any chemical food stimuli [74,126128,141,146-157,166]. Several considerations are needed for the design of electronic taste, including the use of cathodic (e.g. [128]) and anodic (e.g. [147]) tongue probes, manipulation of current and frequency (e.g. [148]), and location of stimulation on top or below the tongue (e.g. [148]). In contrast to electronic taste stimuli that are easier to deploy although they remain limited in the sensations they can create, with none being observed to support all 5 basic tastes, chemical stimuli require reservoirs of tastants to be ongoingly refilled once used which raises challenges for their deployment in naturalistic settings.

Beside taste, the sense of smell also contributes significantly to flavor experiences [171]. However, in contrast to the emphasis on taste, technologies augmenting the sense of smell for eating experiences have been less explored. Interestingly, the limited research on smell interfaces focused on chemical-based ones, commonly used in conjunction with electronic taste [151,152,156,157], and occasionally with thermal and visual stimuli [151,157]. The intention here was of supporting the construction of flavor experiences without consumption of food, or the consumption of healthier but less flavorsome alternatives. One explanation for the limited focus on smell-based interfaces is that their chemical stimuli tend to consist of foodstuff such as chocolate [92,111] or sports drinks [96] rather than actuators or delivery systems.

Thermal actuators, such as Peltier elements are more common than smell stimuli, with 7 papers using them to deliver warmth or coolness to the area around the mouth in order to influence the perception of flavor $[147,148,151,152,157,166,174]$. The exploration of thermal actuators in our reviewed papers tends to be coupled with that of taste, and future work can focus on its potential to support emotional and communication purposes [200]. 
The visual stimuli for augmenting eating experiences involved mostly Virtual or Augmented Reality (VR/AR) as shown in 10 papers $[7,8,69,106,130-132,175,176,191]$. The input into most of these systems consisted of photos of food (8/10) and less so images of objects such as plates [175], or people physically chewing virtual images of food [191]. The output of these system consists of manipulated images of food through the VR/AR technology, either by themselves or in combination with smell stimuli. This suggests similarities between how AR/VR technologies and electronic taste systems are used, the former drawing on the visual, the latter on the taste modality, with both being extended by integrating also smell stimuli.

More traditional visual augmentation through images on 2D screens was reported in 8 papers $[78,135,137,150,153-156]$. Most of these papers manipulate the appearance of food color to influence flavor perception. For instance, they use yellow, green and white lights to simulate different lemonade flavors [150], multicolor RGB LED modules controlled by the users [135,153-156] to select the color lights for drinks as part of systems supporting electronic taste [135,150,153-156], whilst others used projection of red, green, gray [78], or brown, green purple colors [137] to manipulate lighting onto solid foods [78,137]. The intention was to manipulate the flavor experiences to become more pleasurable (e.g. [137]) or to allow swapping to healthier foods whilst maintaining the flavor experience (e.g. [153]).

Audio stimuli for augmenting eating experiences included both ambient sounds or the sound generated by the mouth while eating. 7 papers focused on ambient audio stimuli $[24,44,118,121,178,185,188]$ such as purposefully designed musical soundscapes in order to alter the perception of food taste towards sweeter or more bitter [24]. Two of these systems generated adaptive ambient sound triggered by the foodstuff itself, leveraging the capacitive sensing of food materials such as jelly and ice-cream, that, when touched or licked, triggered electrical signals resulting in pleasurable and playful eating experiences [44,188]. Fewer papers focused on audio stimuli generated while eating $[104,178,187]$. These systems recorded the audio of eating such as the crunching noise of eating crisps [104], and augmented it by varying the volume so when played back it influences the flavor perception towards more crunchy (higher volume) and less crunchy (lower volume), supporting both more and less pleasurable experiences, respectively.

\subsubsection{Smart tableware, tracking apps, games \& automatic dispensing technologies for healthy eating}

An important finding is the prevalence of technologies supporting healthy eating as the most commonly target application domains. These included smart tableware $(20$ papers $)$ from augmented plates $[27,28,52,53,68,83,100,114,158,165]$, to cutlery $[20,29,75,83-86,101]$ and cups $[68,86]$, or the table itself [122]. The augmented plates, cutlery, and cups tracked the eating interactions in order to provide feedback in two main forms. The first is via haptic- or light-based systems triggered by eating too quickly or too much, i.e., [101], while the second uses eating behavior as input via sensors in the forks or plates that measure the consumption of food, for example through video game played at a collocated tablet, i.e., [29]. Augmented tables were also used to regulate eating speed [122].

Healthy eating is also supported through technologies intended to increase users' awareness of, and even regulation of their bodily responses during eating through self-tracking apps [17,19,38,52,53,68,75,83$86,100,101,158]$, and/or games [17,81] (16 papers). An interesting use of sensors in games are ingestible sensors controlled by the gut's temperature [17] to support its increased awareness and regulation. 
Healthy eating was also supported through technologies for automated dispensing, as new form of controlled delivery of usually small amounts of foodstuff intended to foster increased awareness of bodily responses during eating, and their regulation. These were described in 9 papers [90,94-96,123,125,180-182] addressing also the emotional or playful aspects of eating experiences (3 papers), as well as the cognitive ones, most often through reflection on data edibilization (3 papers). Most commonly, such technologies involved pumping liquids such as tastant solutions (e.g. sugar dissolved in water [182]), or drinks (e.g. isotonic sports drinks [96]), with 1 paper reporting dispensing of solids such as chocolate balls [90]. 3 papers explored how the performative mixing of different liquids could be used to communicate data about user's physical activity such as heartrate [94-96]. One of the advantages of liquid foodstuffs is that they can be pumped through a controlled delivery, i.e., measured small volumes. This allowed systems to use 5 different stimuli; one for each basic taste of bitter, salty, sour, sweet and umami [181,182]. The most common purpose of these technologies was using food as part of output of interactive systems supporting ludic eating experiences through gaming [123,125,181,182], entertainment through films [180], or to edibilize heartrate data [94-96]. Pumped liquids such as tastant solutions or drinks were either delivered directly into the mouth while allowing the hands to be used for another activity like gaming $[123,125,181,182]$, or into a cup [94-96]. The pumping into a cup also allowed a performative element to be designed into the experience for instance via a fountain-like device which mixed the drink for the user [94-96].

\subsubsection{2D/3D printing, laser cutting, acoustic levitation and magnetic field technologies for static and dynamic food forms for dining; Video conferencing, camera tracking, and $V R$ for remote dining}

Beyond the sensory experiences with food, technologies have been also used to create a range of novel food forms for dining purposes, both static, i.e., printing (21 papers), laser cutting (3), and dynamic (3). Most such systems relied on food printing through static forms, either as 2D low relief (20), 3D structures (7), or both (6). 2D printing [3,82,95,100,101,122,130,133,139,140,148,151-154,162,188,193-195] has been often used to draw messages by remote dining partners on food such as toast, or jelly-type [3,195-197], or to create visualizations of bodily data for instance physical activity [92,98,99,124]. Similarly, 3D food printing was also used for communication of data such as a person's CV or physical activity $[142,143,192]$ in foodstuffs as jalebi (Indian fried batter sweet) or marzipan (European almond-paste sweet). 3D food printing technology explored variations in taste experiences in solid foods whilst controlling other aspects of flavor such as appearance, smell and texture, thus moving away from the traditional use of liquid tastants for taste-based interactions. Further exploration of novel static forms relied on accessible Computer Numerical Control (CNC) technology such as laser cutting to draw messages onto food surfaces for communication purposes [73], 3D printing of molds to create personalized recipes [203], or digitally controlled cotton candy machines for rapid prototyping in food [67].

Acoustic levitation was also explored as a way to 'float' food in space, as novel forms, not static but dynamically changing in response to user's interaction. A common theme reflected in 3 papers explored the use of magnetic fields and foodstuffs to create performative experiences with food forms where food appears to move in space, either on the plate on in midair which in turn adds a ludic, entertaining dimension to eating experiences $[1,2,144]$.

An important finding is that most of the systems supporting the social aspects of eating experiences did so by supporting remote connection (10). Such technologies included video conferencing, camera tracking, and VR integrated with sensory augmentation and novel food form technologies in order to facilitate remote commensal 
experiences. These papers relate to the broader HCI body of work on commensality technologies $[32,49]$ where video conferencing tools have been commonly used to share sensory experiences of remote co-diners. The distinction is that the 10 papers identified in our review focused primarily on eating experience, so that the technologies they employ are not only video conferencing tools but rather technologies supporting the delivery of novel food forms or food messaging during remote dining in order to share augmented multisensory experiences of flavors as shown in 5 papers. Such technologies involved 3D food printers [3,194-197], as well as haptic/thermal actuators triggering thermochromic ink in a special tablecloth to change appearance while allowing the drawing of images and text between dining partners [3,194-197]. The remaining 5 papers drawn on electronic taste for sensory connection between co-diners $[147,150,166]$, extended video conferencing with VR technology for more immersive experiences of co-dining [106], or social dining robots to support commensality for solitary diners [93].

\subsubsection{Robotic technologies for assistive dining}

This group of 4 papers focused on technologies such as robotic arms that move food from the table to the mouth for people with physical disabilities [82,105,110], or in rehabilitation following physical injuries [176]. Relevant to this application domain of assistive dining, we can consider game-based applications of the same robotic technologies supporting co-diners to feed each other by using robotic arms $[119,124]$. While these applications supported playfulness within the dining domain, they could also be beneficial for assistive dining domain.

\subsection{Discussion of Systematic Review Findings}

We now reflect on these findings focusing on the first Research Question of how eating experiences were leveraged, and designed for in the reviewed HFI research. Our specific focus on eating experiences aimed to shed light into the less explored area of 'design-with-food' and how it complements the more explored one of 'designaround-food' [57] to identify the main gaps or limitations, and opportunities for future work pertaining to eating experiences.

The review findings indicate that the bodily experiences of eating are sensory rich, stimulating both external senses of sight, sound and touch, traditionally used in HCI, as well as the less explored senses of taste or smell $[54,140,181]$, and even less so, the particularly interesting internal senses of digestion and metabolization [17,57]. The use of taste for augmenting user experience is an exciting design opportunity, however more work is needed to understand how it can be effectively leveraged to support users monitor or understand multiple data sources simultaneously (i.e. audiovisual data via taste), and it remains to be seen how appropriate taste may be in this scenario. While most of the reviewed work has explored the leverage of individual senses predominantly through electrical stimuli for unimodal experiences, an emerging trend is the exploration of multisensory flavor experiences through the actual eating of food or drinking of drinks. Also interesting, is that taste appears to be the only sense stimulated both electrically and chemically [151], with most work focusing on sweet, bitter, or sour taste, less work on salty or umami, and even less work on their combination. The focus on internal senses highlights the inside of human body as new site for interaction; including the mouth for licking and chewing, or the gut for digestion [17].

Findings also indicate that the reviewed HFI based systems aim to support sensory, emotional, cognitive and social aspects of eating experiences. With regard to the stimulation of sensory aspects, an interesting outcome is the rather limited focus on sensory augmentation considering explicitly the dining context for table-based eating experiences $[20,197]$. This perhaps indicates the challenge of designing interactive flavor augmentation systems 
without disrupting the dining experience itself. Two other interesting outcomes regarding flavor include its predominant use to support pleasurable experiences, while its value for 'interesting experiences' or even challenging ones [180] has been less explored. For instance, integrating sensory information in surprising ways, i.e., crunchy soup, can be further explored in order to extend beyond pleasure, towards uncomfortable experiences [15].

Moreover, most work supporting flavor experiences has involved generic, rather than personalized foodstuff. This is surprising, given the role of individual differences in the sensory experiences in general, and of tastes in particular [12], suggesting an untapped potential of future work to better support them. For instance, emerging work has shown the value of novel design methods such as sensory probes to support the co-design of personalized flavors, tailored to users' specific needs [59], or of personalizing 3D printed food structure for increased perception of satiety [112].

Besides food or drinks, the stimulation of flavor experience has been further augmented through environmental stimuli delivered in dining context such as ambient sound, especially music, or colored lights. Once again, the potential for personalization or user's agency over such stimuli has been less explored, for instance to support them to deliberately construct the audio experiences rather than merely triggering the playback of the audio track. We could imagine how such audio tracks could be explicitly selected by users, much like wine pairing for a specific dish.

While flavor-based systems have been explored mostly for their own end [24,118,127,137,174,178], emerging work has started positioning them in social dining contexts $[194,196]$ thus, sharing similarities with technologies for commensality [48]. This indicates that flavor experiences can be appreciated in isolation, but also within broader social contexts. It is likely that, as such flavor-based technologies mature, we will see such systems focusing on sensory aspects increasingly integrated in social contexts.

Apart from the sensorial aspects of eating experiences, their emotional ones such as playfulness have been also explored through both formal and informal eating practices, either as structured or open ended games. This outcome is important, as playful food interaction has been limitedly reviewed in HCI [30]. Unlike papers focused mostly on sensory stimulation, those focused on playfulness $[189,190]$, especially open ended games, allow for user's agency and experience of discovery, particularly through creating new sounds while eating. While tastes such as sweet/bitter have been more commonly used to provide positive/negative feedback in games [123,125,182], other tastes and their emotional associations have been less explored. Tastes however have been used not just for triggering emotions, but also for communicating [55,56,123,125,139,182] or even regulating them as part of personalized flavors [56]. Particularly interesting is the innovative exploration of internal senses involved in playful eating experiences. This has been explored through novel game-based systems, usually VR ones aimed to increase awareness of digestion [7,8] and metabolization [94-96]. Such systems open up an exciting design space integrating insights from body-centric design [4] to support such interoceptive experiences and their awareness $[4,76]$.

In contrast to sensory and emotional aspects, the cognitive aspects of eating experiences have been less explored, and when they were, the main focus has been on data edibilization aimed to support engagement and reflection. Relevant here are the food forms, i.e., 2D or 3D printed [92,98,99,124,142,143,164], and particularly data content, i.e., most often bodily data such as tracked fitness data, food nutritional data, and less common, abstract data. Data content could also be foodstuff itself, i.e., usually sweets as reward [92,98,99,124] or sport drinks as refuel [94-96]. In contrast to data edibilization, a less explored cognitive aspect of eating experiences has focused on capturing and communicating stories reflecting autobiographic food $[16,20,45,67]$ that can take on performative qualities when communicated to broader audiences $[20,69,180]$. 
Finally, the social aspects of eating experiences include commensal, cooperative, and communicative ones for supporting social bonding $[105,106,136]$, technologically mediated eating experience requiring collaboration among co-diners $[8,119,122,136]$, and the transmission of often emotional messages $[116,134,154]$ usually on printed food [194,196,197]. Such systems open up new design opportunities for public-private communication where the messages embedded in food, although publicly visible, their rich multisensory qualities can only be privately experienced through eating.

Apart from supporting sensory, emotional, cognitive and social aspects of eating experiences, findings also reflect how these aspects are leveraged across three application domains. An interesting outcome is the increased prevalence of papers addressing healthy eating, over two times more than those focusing on dining. Healthy eating domain has targeted both children and adults, predominantly through persuasive games, and solely adults through smart tableware, automatic dispensing technologies and those for self-tracking of eating behaviors and even regulation of cravings. Tracking focuses on the foodstuff and takes place via cameras [106,113], scales [158], capacitance [68] or audio recordings [8] reflecting the multimodal nature of eating experiences. Interestingly, such systems could benefit from more critical reflection on the goals they support and their tailoring to users' needs. Indeed, previous HCI research on the ethical implications of affective health technologies in general [167], and dieting apps in particular has indicated their potential harmful impact of supporting inadequate relationships with food [45]. The focus on reflection, and especially regulation of eating behavior, has been less explored, with some recent work targeting such important functionalities [90]. The emerging HCI interest in mindful eating offers exciting opportunities to extend the design space of HFI systems, by supporting users' agency to frame their own healthy and mindful eating and how its meaning evolves across contexts $[20,100,101]$.

The dining application domain focused on support for remote dining or co-dining experiences with robots as companions. In both cases, the previous HCI interest in commensality research [36,51] has been extended to integrate both the social aspects of design-around-food, as well as the sensory aspects of design-with-food. While the former has been explored through traditional technologies such as video conferencing, camera tracking, or VR for remote dining, the latter has been explored through more novel technologies from 2D/3D printing or laser cutting, to acoustic levitation and magnetic field in order to deliver new food forms during dining, both static and dynamic. Such exploration of new ways of making and delivering food draws from lower prices and ease to use manufacturing technologies. One can imagine how the integration of knowledge about food and eating experiences within the maker movement can further extend the design space of these technologies and their democratization.

Also related to the dining application domain, another interesting finding is that about a third of the papers support performative experiences, although, as of yet, they have been limitedly explored in real dinning settings and their social interactions. In the dining domain, findings indicate the potential technological challenge of disrupting the dining experience, especially through technologies augmenting the sensory aspects of eating experiences, or through the more invasiveness head mounted VR/AR technologies [8,106,113,129,177]. Interestingly, technologies in dining domain allowed for a range of manipulations impacting the dining environments, albeit less so of the food itself, despite the focus on delivering novel food forms. This may be because the foodstuff per sei may be more difficult to manipulate, as a 'living' material that degrades and changes over short time interval [140]. As technologies mature, we will probably see more of the sensory augmenting technologies integrated within the dining domain.

Not at least, the third, less explored domain focused on robotic technologies to supporting assistive eating experiences of people with physical disabilities $[82,105,110]$ or those in rehabilitation following physical injuries 
[176]. Such work attempts to model 'normal' eating behavior informed by abled bodied, that is not sufficiently critiqued ]78].

\section{STUDY 2: INTERVIEWS WITH PROFESSIONAL CHEFS}

The aim of the second study was to explore chefs' creative design with food and particularly their focus on aesthetically rich eating experiences. In contrast to the first study, the interviews provided further depth to the 'what' and 'how' of designing for eating experiences through complementary rich accounts of professional chefs. As practitioners, they engage in creative design for eating experiences that are likely to share novel, pleasurable and aesthetic aspects. The interview study aimed to address the second Research Question.

\section{$3.1 \quad$ Method}

We ran semi-structured interviews with 18 professional chefs who had at least 5 year experience (range 5-30, $\mathrm{M}=$ 12.67, S.D. = 5.71), including 8 food designers. The average age of participants was 34.8 (range 29-55, S.D. = 6.2) 8 participants identified themselves as female, 9 as male and 1 as non-binary, representing a gender-balanced sample. 11 participants identified as British, and one each as Canadian-British, Czech, French-Colombian, French-South African, Greek, Icelandic and Nigerian-American. Participants were recruited as they are directly involved in the design and creation of eating experiences with handmade food, having also significant practical expertise that they can draw from to share with us. The contexts in which they work are diverse 9 worked in restaurants, 7 in catering for food events such as theatre performance, or films), 5 in producing food art for events and exhibitions, and 1 in food product development where recipes were designed for clients including restaurants and food brands. 2 chefs worked in more than one of these contexts. Participants' selection was made to combine both traditional approaches to food experience, as seen in restaurant settings, with more experimental experiences found in the practice of those chefs who were also food designers. All participants were recruited from the UK via mailing lists associated with Dovetailed Ltd.

Through the semi-structured interviews we asked participants about the qualities of foodstuff such as flavors, tastes, forms, textures or other factors that they manipulate in their creative process. We also asked if factors regarding the environment in which the food is eaten are also manipulated and how. Participants were also prompted to reflect on whether they designed mostly for positive experiences and also for challenging or uncomfortable experiences, and how each of these may feel like. The study also involved an additional task where without being given any tastants, participants were asked to recall typical food experiences of each basic taste, i.e., sweet, sour, salt, bitter and umami, and to place them onto the Russell's circumplex model of affect [163] representing a two dimensional space organized along arousal or intensity axis, and valence axis, i.e., positive/negative. While traditionally, this model has been used to place discrete emotions such as love or hate within the space, previous work [58] has shown that it can be also used to place the experiences of each basic taste by matching them to the discrete emotions people felt that best represented each taste. Interviews lasted around 45 minutes, were audio recorded and fully transcribed. These were analyzed using Atlas/ti software, through a hybrid approach including both deductive and inductive coding [50] leading to a final set of over 200 codes. The deductive codes were identified from theory-informed conceptual framework inspired from previous work on taste-emotion mappings [18,161] to highlight tastes and emotional responses, including potential use of metaphors [25], the multisensory nature of flavor perception [171] to help uncover both the sensory cues arising from the food 
as well as the context in which the food was consumed, uncomfortable experiences [15], and trajectories [14] to examine both negative or challenging food experiences as well as approaches to mapping out experience over a period of time as series of interactions. Then we revised this framework on the basis of interview data as new codes emerged around chefs' process to design food experiences such as balance, complexity, ambiguity, transformation, ideation, iteration, and movement metaphors for in-mouth sensations such as kick, burst, kick, lift, pop. The themes were iteratively refined through discussions over 3 months between the two authors until consensus was reached.

\subsection{Findings and Discussion}

We now report on the four identified themes describing emotionally rich sensory experiences, performative aspects of eating experiences, memory-inspired design of eating experiences, and chefs' approach to designing for such experiences. We also discuss the importance of the key findings in the light of the second Research Question of how professional chefs design for eating experiences and their aesthetics. While some of these findings confirm those from the systematic literature review, others extend them thorough the more nuanced understanding of taste-emotion mapping leading to flavor-emotion mapping, innovative uses of taste, smell or visual stimuli not only for augmenting pleasurable eating experiences but also to inject challenge and tension, novel haptic in-mouth experiences and internal ones in the gut, the cultural aspects of eating experiences, and the framing of eating experiences not just as pleasurable but as purposefully designed trajectories to support climax through contrasting and balancing of pleasurable and uncomfortable experiences.

\subsubsection{Emotionally and Sensory Rich Experiences: Taste, Smell, Sight, Touch and Internal Senses}

A key finding is chefs' deliberate effort to design for emotionally rich multisensory flavor experiences, while leveraging the main senses of taste and supporting its appreciation. While the connection between taste and emotion has been consistently shown in previous HFI research $[62,89,161]$, our interviews have unpacked more nuanced understandings of such mappings. In particular, as shown in Figure 2, all the four tastes from the top right quadrant were associated with mid to high arousal positive emotions, while the salty taste to the lowest arousal.

\section{High Arousal}

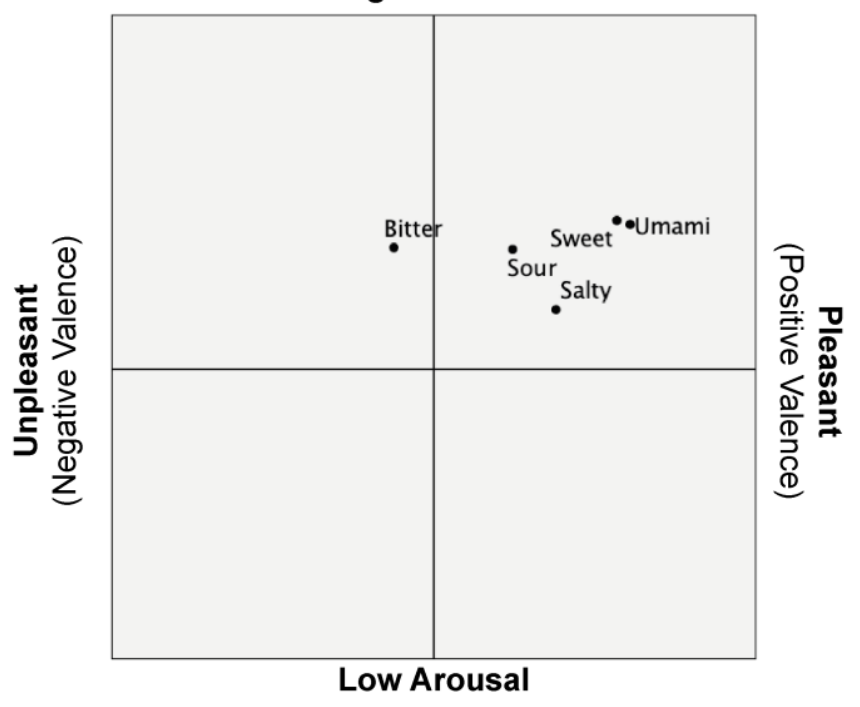


Figure 2 Chefs' mean ratings of emotional valence and arousal associated with each of the 5 basic tastes

Participants appeared to prefer tastes of medium-high intensities $(n=8)$, confirming previous findings [55]. Findings also show larger variance in the mapping of the salty and sour tastes which were the ones associated to both positive ( $n=9$ for salty, $n=11$ for sour) and negative emotions ( $n=8$ for salty, and $n=14$ ), where the numbers in the brackets represent the number of chefs supporting them. For instance, sour taste was often associated with positive emotions through recall of eating sour sweets in childhood, contrasting previous findings that solely mapped sour tastes to negative affect [62].

Another important outcome is chefs' nuanced understanding of tastes beyond taste-emotion mapping, and in particular through the dominant characteristics of multisensory flavor experiences. For instance the sour sweets provide not only a sour taste but also a less dominantly sweet taste, with specific texture and appearance. This suggests the value of extending taste-emotion mappings to complex flavor-emotion connections where specific tastes are dominant while others less so. For instance, the connection between bitter tastes and bitter emotional state has been previously made [26], and also mentioned in our interviews. Moreover, sadness was associated with salty taste: "sadness, tears, they're salty" (P3) or with the description of fear as "really nasty cold coffee not the nice drip stuff, something that has been sat around for too long, that has maybe had a bit of fag (cigarette) ash in it" (P8). Sour or bitter tastes were reported as having an impact on the chemistry of the experience as palate cleanser $(\mathrm{n}=$ 5): "we will use more sour or bitter flavors as a palate cleanser or just to create a kind of a space or a gap before a major sweet or salty flavor" (Participant 13). Similarly, fat was also reported by 4 participants as "carrying the flavor so then other elements [of taste] can come in" (P16). Additionally, umami was reported as a complex taste (n=3) supporting uncertain or intriguing experiences. Findings also suggest that non-taste characteristics such as trigeminal stimulation from spicy foods $(n=13)$, burnt materials $(n=7)$, or very sharp textures such as dry puffed pork rind $(n=6)$ can also be mapped to emotional responses such as happiness, sadness, and anger, respectively. Chefs also described how the temporal qualities of taste (reflected in [139]) can be designed with, as part of the structure of an experience deliberately creating a 'little pop and it is something that takes you by surprise and wakes up the palate' (P2).

In addition to taste, smell was the second targeted sense, discussed by 11 participants for its role in eating experiences. Largely, smell was considered part of the multisensory experiences, as aroma in flavors both compliments and contrasts the taste $(n=4)$. The latter is an important finding, extending the value of smell from the reviewed HFI research. For instance, participant 9 described creating a dish inspired from a scene at " $a$ rotten fish market" in which he "wanted to evoke some of those [rotten, fishy] aromas whilst still making it taste good". Here, smell was explored to create tension between what is perceived by the mouth and the nose, delivering pleasure to one and disgust to the other. Nevertheless, the taste remains the dominant sense, and the appreciation for "good taste" is an important outcome. Smell also interacts with the body at a greater distance than taste; Participant 7 reported covering a bowl of soup with a biscuit to preserve the intensity of smell until the diner lifted it, or to waft a smell prior to serving in order to play with diner's sense of anticipation. These findings are interesting, suggesting that in comparison to literature review's outcomes, chefs were more deliberate in their use of additional smell stimuli, by playfully creating challenges or misdirection, rather than merely stimulating or augmenting the flavor experiences. The use of a biscuit to control smell delivery also suggests novel opportunities for HFI to explore approaches to design for eating experiences that support the controlled release of smell during interaction. 
Visual stimuli, and in particular colors have also been shown to impact on taste perception [172], and our findings confirm chefs' appreciation of this sensory modality. More abstractly, color played a role in the associations of tastes and emotions as reflected in the answers of 8 participants: "I associate anger and rage with the color red and chilies, [they] are very hot and fiery" (P5). This is interesting, as it extends the taste-emotion mapping with specific visual stimuli, which has been limitedly explored in HFI. Participants also discussed the role of color in challenging familiarity and generating surprise, for example through the use of black foodstuffs: "this sticky, oozy, beautiful, black syrup, liquid thing [...] a jelly thing and it tastes like fish but you're like, 'what is it? Is it squid ink?' [so one experiences] emotions of surrender and fear and anger and these feelings of survival [...] your taste buds are aware that it is food [but not] food as you know it" (P17). In this illustrative quote, color stimulus appears to contradict the taste stimulus, in order to create an estrangement with the food, which in turn could capture attention to otherwise mundane eating experience. Here, the appreciation for the taste of the food is reflected in the aesthetic quality of the "black syrup". Beside color, the visual appearance of the food was also used to misdirect, but unlike VR/AR applications which trick users into seeing more pleasing (i.e. bigger/sweeter) food, chefs tricked participants into seeing a less pleasing food. In this respect, our findings uncover new opportunities to design for eating experiences as chefs aimed less to support consistent flavor experiences in which the visual appearance improved the overall flavor, but rather to support those experiences where the visual appearance also challenged the flavor experience which becomes revealed only through eating. An interesting outcome is the chefs' limited mention of leveraging sound in their design for eating experiences, with hearing sense being also limitedly explored in HFI $[104,178,187]$.

Beside taste, smell and sight, chefs' were also interested in touch and its relation to internal sense of digestion. Findings indicate that the sense of touch in the mouth was deliberately elicited through the texture of foodstuff: "really quite dry puffed pork rind, it's sort of a savory honeycomb texture and it is quite sharp and if you try and eat that it will scratch your gums up a bit which is quite unpleasant" (P8). The physical interaction between texture and the mouth opens up new perspectives on HCI design, adding to the spiciness that "sets your tongue alive" (P17) and sourness where the face "all crunches up" (P17). These quotes illustrate the appreciation for eating experience due to the unusual texture or the intensity of the flavor or taste experience.

The focus on digestion is also reflected in the verbs describing the "movement" of taste within the mouth, especially for the sour taste ( $n=4)$ which "bursts", "kicks" or "pops". Most haptic-related eating experiences in our systematic review involved thermal experiences on the hand e.g. [197] or mouth e.g. [174], with little exploration of in-mouth experiences. In contrast, the chefs detailed how the in-mouth experiences can result in pain or trigeminal sensations. While digestive and metabolic experiences have been less explored in HFI, with only one paper in our literature review explicitly focusing on gut [17], our interviewees indicated the interest on both gut and mouth as relevant for digestion and metabolization.

\subsubsection{Performative Aspect of Eating Experiences: Trajectories, Climax, Pleasurable-Uncomfortable, Balance \& Contrast}

An important finding is chefs' interest to design food experiences as trajectories intended to support not just positive eating experiences but also climax through contrasting and balancing such pleasurable and aesthetic experiences: "it needs to be pleasant [...] I have to find joy whilst I am eating it [or] I am not going to serve it" [P14], with uncomfortable experiences: "if you break the rules a little bit with what you can do with food then it opens the door to all those other emotions" (P17). A key aspect of such contrast is the element of surprise that challenges expectations, which literature review findings indicated that are important aspects impacting on eating experiences $[35,37,172,201]$. As mentioned by our chefs, most dishes can accommodate such contrast: "I mainly work with 
desserts [...] no one expects to be made fully from sweets so you can be very playful with them" (P6). This quote suggests that contrasting flavors may be not only possible, but desirable, and a creative dish with surprising elements can positively challenge expectations.

The term trajectory relates to different eating experiences while eating a dish or multi-course meal: "Mugaritz [experimental restaurant in Spain], stands out [...] where not everything is immediately pleasurable [...] it's a sort of emotional, psychological journey to take people on. And I think that is where the concept leads over flavor and deliciousness" [P7]. Trajectories also involve various points that raise to a peak or climax, as a turning point from where to return to lower intensity: "A meal for me is a journey, you want to start with something which is going to inspire your palate and evolve into building up to a richer, heartier main course and winding down; Taking your palate on a journey from savory and bitters flavors and finishing off with more sweet than sour at the end" (P3). This echoes Benford and colleagues' work on framing user experience as interaction trajectories [13,14] that builds on dramaturgy concepts of story, plot, character, audience, space and time from Performance Studies. According to them, user experience is characterized by sensations, positive emotions, and aesthetics, while trajectories are continuous and coherent, capturing the unfolding of successive experiences through time and space. Our findings extend such framing with the dramaturgical concept of climax; the inflexion point in the trajectory of the narrative marked by the greatest tension, as indicated in Freytag's analysis of dramatic composition [65].

Findings suggest four strategies employed by chefs to design for climax within eating experiences and their trajectories. These all rely on leveraging taste-emotion mapping in order to elicit predominantly positive emotions, albeit counterbalanced with negative ones at the climactic moment. One strategy focuses on the predominant taste stimulation during climax, with findings suggesting the value of umami and bitter tastes to provide such tension: "umami can be a fitting high point to a meal, so we probably work up our taste, flavors and dishes around that main kind of high point" (P13). Another strategy involved experiencing less pleasant taste followed by a positive one. For example, sour taste was reported as having initially negative response that changed to positive: "sourness has that thing where first of all it repels you, but then you want more so it's a kind of naughty but nice kind of sensation" (P10). This outcome confirms previous one, with Obrist and colleagues calling this affective-temporal aspect of sour taste as synchronic duality in affect [126]. Another example is the miracle berry, a chemical technology for manipulating how foods taste after being eaten. By blocking specific receptors or inducing salivation [204], it can be used to transform raw lemon into a sweet tasting delight (P11). This is an interesting outcome, as much research on HFI has focused on stimulating taste, but less so on how to prime the mouth by blocking receptors. The third strategy explores contrast across modalities, for instance through the pairing of pleasant tastes with unpleasant smells, in order to design for a challenging but ultimately enjoyable experience: "I think there is something about giving something to someone to eat that is not nice, that seems very cruel, whereas smell is something that you can't control how much you smell things, it invades your nostrils. I suppose there is something quite a bit softer about that" (P6). The fourth strategy involved making the environment more challenging through zombie smells (P6) or sensory deprivation, i.e., BDSM-inspired, blindfolded feeding (P11). Most HFI work has focused on pleasant flavor experiences, or on flavors to support pleasurable emotional, social or playful experiences. A noticeable exception is negative valence taste-emotion mappings used alongside positive one as feedback in games, albeit future work can focus more on how uncomfortable experiences can be also leveraged for designing interesting user experiences. 
When discussing uncomfortable experiences, balance was highlighted as a key consideration ( $\mathrm{n}=8)$, most often in relation to the taste of a dish. For example: "adding in a very bitter element somewhere in a nice creamy mayonnaise or something to give you a nice range of sensations" (P1). Acidity, sourness, and umami were all reported as important in creating dishes with balanced flavor. Balance however is important not only for contrasting pleasurable-uncomfortable experiences, but also for cohesive stimulation across multiple sensory pathways such as taste, smell, and touch: "fish and chips [...] has contrasts of taste and temperature and texture [...] the crispy batter of the fish, the soft flesh of the fish, the comforting texture of the chips and I try and use that as a model for my own dishes" (P3). For both balance and contrast there is a recognition of the need to pair together differences to create interest within the dish and across multiple sensory pathways, albeit within a coherent overall experience. For example, taste, texture or temperature can create interest across a multi-course meal: "one dish is kind of veering towards the sweet, salty, umami side, you kind of go to another dish which ends up on the more bitter, fattier side if that makes sense. So, it is all about trying to create total balance from beginning to end" (P16). These findings reflect chefs' understanding of how temporal experiences can be shaped both within conventional meal structure, as well as in more open ended contexts. Temporal experience around eating in HFI has been limited to taste related experiences [139] and eating speed [122,136]. Chefs connected these temporal aspects of experience to sensory ones, particularly taste. While previous work $[146,166]$ has identified ways in which individual experiences can be augmented, less attention has focused on how different flavor experiences may work together. Our interview findings suggest that contrasting sensory experiences in terms of texture, taste or temperature could strengthened their aesthetics quality.

\subsubsection{Personal and Cultural Meaning of Eating Experiences: Memory-inspired Design}

Findings confirm the importance of eating experiences being highly charged with both emotions and meaning $[102,107,116]$, and that memory plays an important role in the design for, and meaning making of food experiences. For example, chefs' childhood memories were commonly reported, with 11 participants referencing them. As previously mentioned, sour sweets were a common theme in these early life memories. Both positive experiences such as "Sunday mornings as a child, going to the sweet shop with Dad" (P16), and negative experiences with bitter tastes or bad seafood were encoded. Leveraging the memories of these flavor-based experiences by recreating them through novel design of eating experiences was mentioned by 3 participants: "the fun part of being a chef [is] trying to get the customer to understand what you are expressing with that flavor" (P14). As shown in the literature review's findings, there has been limited exploration of food-related memories in HFI, with only one paper directly addressing the potential of food to support reminiscing [61]. Our chefs however described their own, emotionally rich, episodic memories inspiring their design of eating experiences.

Although these flavor-based memories were personal, their association with positive experience appear to hold true for diners, such as the connection between positive experiences and sour sweets, supporting also a feeling of nostalgia ( $\mathrm{n}=5$ ) described as a "secret, powerful influence [of] food" (P6). Key in understanding the meaning of chefs' memory-inspired dishes is the cultural aspects embedded in such personal and shared experiences [108,168]. In particular, familiar, culturally significant food can support positive experiences, while the less familiar food pose some challenges. For instance, chefs with Nordic ancestry described "rotten shark and puffin" (P1) as delicacies, albeit disgusting for diners unfamiliar with Scandinavian cuisine. Licorice was another food eliciting pleasure or disgust. In this respect, familiarity with the cultural aspects is key in shaping the boundaries between pleasant and unpleasant experiences. For example, participant 9 detailed the tension in experimenting with these questions such 
as: "how far can you push it? I suppose sometimes you want to make something that is a provocation and then how far is it acceptable to go beyond something that is straightforwardly tasty; that is a real grey area - really interesting". This is an important outcome as the cultural significance of what is eaten has been limitedly captured in the systematic review findings. Our findings resonate with Korsmeyer's [107] philosophical critique of taste experience arguing for the need to go beyond the refined pleasure underpinning the aesthetic appreciation of taste, to also include the cognitive experience related to personal, social and cultural meaning of food being eaten, as it is through its (usually forgotten symbolic) significance that food resembles arts, a point also highlighted in a growing body of work in food art $[34,102,116]$.

\subsubsection{Chefs' Approach to Designing for Eating Experiences and their Aesthetic Qualities}

Professional chefs mentioned the importance of research $(n=5)$, experimentation $(n=5)$ and iteration $(n=4)$ as key components of their creative process of designing for good eating experiences whose aesthetic qualities matters. They engage in an iterative process to refine the ingredients and recipes in order to shape the experience. Such iterative orchestration of how ingredients fit together for a sensory rich experience echoes user-centered and craft-based design in HCI [22,23], highlighting how food materials can be considered via their experiential outcomes: "I might think of the trigeminal senses, I might think actually could this do with a bit of isothiocyanate (compound found in cruciferous vegetables like broccoli), like the wasabi, pepperiness and stick a bit of horseradish in, or I might taste a broth and think, oh well, it is really nice and umami but it needs a bit more salt, a bit of acidity to freshen it up" [P7]. This iterative process targets not just ingredients but also the smell, sight, and structure of the whole experience: "wouldn't it be nice if [the dish being created] was actually purple so that would build the expectation, so I might think about the smell and [as] I am serving a little cup of broth; do I want to cover the broth with some kind of little biscuit or something so that it only releases the smell once it hits the table or might I want it to waft the smell before I do that, before I serve it, so there'll be anticipation" [P7].

Findings also indicate chefs' different starting points when designing for eating experiences as predominantly flavor-led or narrative-led. For the flavor-led experiences, the starting point is the sensory focus towards designing rich and engaging multisensory experiences: "so [we consider] hot, cold, sweet, sour, hard, soft, black and white; all of those different contrasts you can build into the dishes, to make it exciting to make it fun make it more 'craveable'" (P1). Similarly, P2 noted how he framed his process: "I use a meal that everyone is familiar: fish and chips is a really good one because has contrasts of taste, temperature and texture. Some people might like a pickled gherkin with it, which is really nice and cold and acidic; some people might like brown sauce which adds that fruity acidity and complexity". Narrative-led experiences, while also designed to be sensory rich, are however organized around a narrative storyline, such as the narrative structure of a film, theater performance or novel and its overall trajectory: "we might have a theme we want to run all the way through; so, we start by thinking about the theme and then attach things to it. [We ask] 'what is the big thing?', the main course? And then plan the rest of the menu around that" [P13].

Findings also indicate that professional chefs, irrespectively of their different starting point, consider the whole experience from engaging "as many senses as I can" (P7) to answering questions about the experience such as who?, what?, why? and where? (P12), and its cultural aspects from its history to provenance of ingredients (P1). With respect to the latter, participant 1 described developing a Middle Eastern dish and struggling to get the taste of the meat right: "we were walking through the cheaper sort of areas and [...] we saw all the markets and little butchers and the meat and mutton and lamb hanging out in the sun, so it was getting a bit rancid out in the sun. So, we bought some 
[...] and the feedback was 'yeah, it's great, what did you do? It's perfect!'”. This suggests the importance of understanding the cultural aspects of eating experiences from local clime to food retail.

As shown above, interviews' findings indicate a chef-centric model of design eating experiences reflecting chefs' creative process and personal artistic expression. This model has been critiqued in food design research for limiting diners to the role of passive consumers of eating experiences [199]. To address this, Wilde and Bertran proposed a new method: participatory research through gastronomy design, aimed to engage diners in the co-design of playful experiences [199], while similar novel approaches to co-design of flavors have started to emerge in HCI [59].

We conclude with a reflection on the useful distinction between eating experiences and eating practices, as it becomes relevant for better understanding chefs' approach to design. According to Reckwitz [160], social practices represent routinized behaviors integrating bodily, emotional and mental activities interconnected through the use of specific objects together with implicit, cultural knowledge of understanding such behaviors. Therefore, irrespectively of taking place at different spatio-temporal contexts, practices are easily understood by people, either performing or observing them. A practices is for example a specific form of working, or a form of cooking involving all these elements in a cohesive whole. In his exploration of the complexity and ubiquity of eating practices, Warde [193] called them "compound practices" [p. 24] integrating sensory, social, and aesthetic elements of meals and events whose enactment (or the so called performance of practice) involves many situated instantiations of the practice. Each instantiation means unique bodily, emotional, and mental activities taking place in a specific space and time [160]. These bodily, emotional, and temporally bounded events share strong similarities with the aesthetic, emotional and experiential aspects of subjective phenomena [72] characterizing user experience [70]. Warde [193] further identified four specific practices underpinning the complex eating ones: supplying the food, cooking, organizing eating events involving etiquette, and the practice of aesthetic judgments of taste. Our findings highlight not only chefs' creative design for refined eating experiences, but also their engagement in the specific practice of aesthetic judgments of taste [193]. Indeed, the practice of eating is particularly open to aesthetic judgment especially by those with much appreciation for the aesthetic and experiential aspects of eating, such as professional chefs with expert knowledge and passion for food [67].

\section{GENERAL DISCUSSION}

We now look back to the remaining research question. The first and second Research Questions have been addressed in the Discussion of the first and second study, respectively. In this section we reflect on how the main outcomes from the two studies build on, and complement each other, in order to answer the third and fourth Research Questions of what is the space of designing for user experience in HFI involving eating experiences, and how we can better support its understanding and exploration. In addressing these questions, we articulate three main contributions of our work including a conceptual framework of designing for user experience that leverages eating experiences, as well as the mouth and the gut as new sites for HFI, novel implications for HFI design research leveraging sensory and emotional aspects of eating experiences, as well as the cultural and performative ones; and six charts as novel inspirational and generative design tools for HFI experiences.

\subsection{Conceptual Framework of Designing for User Experience informed by Eating Experiences}

First, we start by reflecting on the identified aspects of eating experiences and how they can be conceptualized. As our findings indicate, by connecting the body and technology, food can performs various roles from meaningful data object $[92,94,143,192]$ to prompt for eliciting emotions $[55,56,80]$ or supporting storytelling $[21,42,69,180]$; 
and it does so flexibly through flavor [55,56,125,182], shape [92,143], structure [44,67] or form of delivery [183]. More importantly, food's multisensory quality makes it a prime stimulus for multisensory experiences, involving stimulation of taste, smell, sight, sound, touch [10], as well as internal sense of digestion and metabolization.

Research on developing frameworks for designing for experiences has received rather limited attention in HFI, with initial explorations focusing on how taste may support specific temporal, affective and embodied experiences [139]. Such previous framework however has been developed based on the mere exploration of taste experiences involving tastants rather than flavors, and does not capture the richer bodily experiences involving both internal and external senses, or the cognitive aspects of eating experiences.

In order to harness the value of eating experiences for designing for user experience, we refer to the seminal work of Hassenzahl and Tractinsky [70] who, although have not provided a definition of user experience, identified three key dimensions: (i) aesthetic, hedonic and holistic that extend beyond mere instrumental functions, (ii) subjective and positive emotions, and (iii) experiential aspects such as complex, dynamic, unique, situated and temporally-bounded. Another relevant model is the HCI framework for experience [51] and its concepts of experience, (i.e., constant stream), an experience (with start and end), and co-experience (ensuring emotion and meaning). For example, the constant stream can be seen as bodily engagement during multi-course meals, an experience may involve tasting a simple tastant and perceiving its ephemeral impact in one's mouth [47], while coexperience may consist of co-dining involving emotional or storytelling aspects [45]. This useful framework accounts well for the emotional, cognitive, and social aspects of eating experiences, but less so for its explicit sensory ones. So an integration of the two frameworks above would be useful. Moreover, as reflected in chefs' interviews, eating experiences also appear to share performative aspects which can be accounted for if we employ the framing of experiences as interaction trajectories $[13,14]$ together with Freytag's analysis of dramatic composition, i.e., climax, contrast, balance of pleasurable and uncomfortable experiences [65]. Such uncomfortable experience echo "difficult pleasures" such as disgust, fear or sadness that Korsmeyer [107] described as deeply moving, yet challenging, albeit contributing to heighted appreciation of flavor.

Not at least, our findings suggested the value of the body in the rich sensory, emotional and meaningful experiences associated with eating. Similar qualities related to food as a medium for arts [107] and artistic performance [34] have been suggested in research Performance Studies: "Food, and all that is associated with it, is already larger than life. It is already highly charged with meaning and affect. It is already performative and theatrical. An art of the concrete, food, like performance, is alive, fugitive, and sensory" [102:1]. This quote illustrates the sensory, emotional, communicative, performative, as well as temporal aspects of eating experiences. With respect to bodily aspects, our outcomes also highlight the mouth and the gut, as two less explored sites for HFI, and potentially for HCI. To better design for eating experiences within HFI, a closer look at these bodily spaces is needed. For his, we build on a rich foundation drawing from phenomenology and the post-Cartesian philosophy [120]. In particular, somaesthetic design has emphasized the role of the body in user experience, and the value of cultivating aesthetic appreciation of the bodily experience through guided attention inwards [77].

\subsubsection{The Mouth as Site for HFI}

Our findings highlight the importance of the mouth as a novel site for HFI. The mouth performs many roles on eating experiences, from evaluating the food [10] to breaking it down into smaller parts. Indeed, digestion starts in the mouth through chewing or licking. The mouths is also key in the construction of the multimodal sense of flavor [91] integrating taste, smell, touch, i.e., texture and temperature of the food, and trigeminal sensations. The mouth 
is also key for the experience of emotional aspects associated with specific tastes and flavors, the most predominantly designed for being positive ones such as pleasure, enjoyment, or delight. Findings indicate chefs' focus on designing also for uncomfortable sensations in order to ensure performative eating experiences by contrasting them with pleasurable ones. For this, we have seen the importance of umami and bitter tastes, as more challenging tastes to be followed by pleasurable ones; of pairing unpleasant smells with pleasant tastes for climactic moments; or of using palate cleansers as pauses in the trajectory of eating experiences. From a temporal perspective, the experiences in the mouth are mostly ephemeral [44] engaging tastes, textures and temperatures, but they can also be designed to linger through sensation of hot, following spicy flavors that simulate damage on the sensitive flesh of the mouth. Chefs' interviews also indicated that the range of interactions in the mouth is rich, going beyond the mere "chewing" and "licking" that have been mainly explored in HFI, to more nuanced ones such as "bursts", "kicks" or "pops" reflecting the movement of the taste in the mouth, especially sour. The exploration of the embodiment of taste experiences has been limited, with previous findings indicating that the feeling of the mouth due to different tastants may be linked to their texture, viscosity, or movement in the mouth [126]. Efforts to develop richer vocabulary for these in mouth interactions and how they feel like can further advance the field.

\subsubsection{The Gut as Site for HFI}

Our outcomes also highlight the importance of the gut especially for internal senses of digestion and metabolization, and subsequently as a novel site for HFI. Unlike the sensations in the mouth, those from inside the body are less accessible, although people can recognize and learn to understand them [5,72]. Findings from experiential phenomenology identified the importance of the body and especially the gut for intuitive feelings [71] which may be brought into awareness as the "felt sense" through Gendlin's focusing method [60]. The gut, as new site for HFI, draws from this richer background of the body as site for knowledge production, especially since sensations inside the body such as fullness, hunger, satiety, warmth and their emotional aspects have been less explored in HFI but can offer exciting opportunities for design, especially for healthy eating domain [17]. Increased awareness of such digestion and metabolization processes can help reshape relationship with food. For instance, novel applications such as those supporting mindful eating [20,100,101] can increase people's awareness of such feelings and potentially their ability to better regulate eating behaviors. From a temporal perspective, unlike the short-lived experiences in the mouth, those inside the body are marked by larger time scale. While the mouth provides instantaneous feedback such as positive emotions triggered by sugary treats, their digestion can take several hours after which they become metabolized in some form of fat. Exploring ways to support awareness of bodily experiences of eating across these different timescales can open up novel design opportunities.

\subsection{Implications for HFI Design Research}

The two sets of findings from the two studies presented above share similarities but also complement each other. The key outcomes about how together these sets of findings define the design space of HFI are further described, alongside insights on how future research building on them may move the field forward. While our literature review identified the sensory, emotional, cognitive and social aspects of eating experiences, the interview study also

highlighted the importance of performative experiences, and cognitive ones reflecting mostly communicative experiences. 


\subsubsection{Beyond Taste Stimulation: Supporting Multisensory Flavor Experiences}

Our literature review's findings indicated that much research efforts have focused on the sense of taste, predominantly through electronic taste stimulation devices, i.e., [141,156]. A less questioned assumption of such devices however is their potential to support healthier eating, for instance by swapping sugary drinks for water drunk with augmented electronic taste, i.e., [153,154], or by reducing salt content by electronically creating salty tastes [128]. However, such health potential has been limitedly realized, given the reduced acceptability of electronic taste systems and ability to deliver the full range of taste sensations. Indeed, beside sweet and sour which are the most common electronic tastes, others, such as salty and bitter have been less explored through such devices [149]. Other limitations of electronic taste devices include the discomfort of using them, and lingering aftertaste [149] that perhaps explains the shifting trend towards leveraging such devices for communication purposes $[126,147,166]$, especially of affect. Indeed, an interesting potential of electronic taste technology is leveraging the link between taste and emotions $[56,139]$ in order to better support taste-based emotional communication, and to increase the enjoyment of multisensory experiences [126]. We encourage HFI researchers to move beyond unimodal experiences, and towards supporting multisensory ones $[133,134,184]$ which were the main focus of professional chefs in our interview study. Future research could also explore the integration of electronic and chemical stimuli across multiple sensory pathways, since leveraging chemical sense through consumption of food or drinks offer opportunities to elicit multisensory experiences and in turn to better support them for richer user experience.

\subsubsection{From External Stimulation to both External and Internal Sensory Stimulation \& Deprivation}

An important outcome from the literature review is the breadth of sensory stimulation addressing the five external senses. Although HFI research has started to also explore digestion and metabolization, much less work has focused on these internal senses. In contrast, our interviews, professional chefs pay much attention on them, reporting a richer range of interactions in the mouth, going beyond the mere "chewing" and "licking" that have been mainly explored in HFI, to more nuanced ones such as "bursts", "kicks" or "pops". Efforts to develop richer vocabulary of such "in mouth interactions" can further advance the field.

The findings from both studies pointed out, in different degrees, the value of regarding the mouth and the inside of the body, especially the gut [17] as places where interesting things occurs, or in other words as sites for interaction. We suggest the value of integrating both external and internal senses for the design of eating experiences and beyond towards multisensory user experience. This can open up interesting design opportunities supporting also the focus on interoceptive experiences as an emergent HCI interest related to human body [4,76] and how they may be supported [33]. In addition to the distinction between internal and external senses, most of the reviewed HFI research has focused on stimulation, with limited interest in sensory deprivation. However, we have seen chefs' efforts to leverage deprivation in their design for eating experience, which echoes previous findings suggesting its value for intensifying the non-deprived senses, i.e., taste in the absence of sight [151,152]. This opens up new opportunities for HFI exploration of sensory deprivation, and potentially for novel methods supporting it, like recent work on sensory probes [59].

\subsubsection{From Taste-Emotion Mapping to Flavor-Emotion \& Flavor-Emotional Memory}

Eating experiences are quintessential pleasurable, and both studies confirmed previous findings on the relationship between tastes and emotions. However, in contrast to literature review findings, chefs' interviews 
suggested richer and more nuanced mappings. First, there is much value in moving beyond mere tastes but to flavors in order to better harness their relationship with emotions. Indeed, similar to tastes, flavors can elicit, communicate and regulate emotions [55], while the increased complexity of flavor-emotion mapping can support the communication of ambivalent or even ambiguous emotions in the context of user experience, for example by leveraging combination of dominant and secondary tastes. Moreover, the multisensory quality of flavors can be creatively leveraged in such mapping, where for instance anger can be mapped to fiery chilies, and both to the color red. The interview study also highlighted the less explored connection between food and emotional memories, in particular those from chefs' childhood and their value in designing for more engaging eating experiences where emotions and meaning are both harnessed. This less explored connection in HFI between memory and food offers interesting design opportunities drawing from the value of food in emotionally relevant episodic memories and the growing HCI research on memory technologies where flavor-based cues have been more recently explored and codesigned [59], for instance, as personalized 3D printed flavors [57] to support reminiscing in old age [58].

\subsubsection{Communicating Meaning by Leveraging Social and Cultural Aspects of Eating Experiences}

Food experiences are not only sensory and emotionally rich but also meaningful. They often communicate meaning through the social and cultural context in which eating experiences take place. Literature review indicates that such meaning is communicated through edibilization of data pertaining to fitness or food nutrition [68,87,8993], as well as storytelling [20,69,180] in social context $[20,93,106,136,194,196,197]$ where eating experiences were intended to support bonding. Interview study complements these outcomes by showing also the value of memories in communicating stories through food, and of cultural aspects underpinning eating experiences. More importantly, cultural aspects appear to impact diners' emotions and experiences of eating, as they appreciate more the familiar flavors and less so the unfamiliar, challenging ones. These outcomes suggest the value of considering the meaning of food and eating experiences in HFI research from the broader perspective of their cultural aspects, whose tension could be innovatively explored through design.

\subsubsection{Designing for Dramaturgical Climax of (Flavor-based) User Experience}

The design of engaging user experience can benefit from additional performative qualities, and literature review findings indicated efforts to support these through playfulness, entertainment or surprise, elicited for instance through novel technologies such as those "magically" manipulating food through magnetic $[1,2,144]$ or acoustic levitation [181,183]. While such focus on HFI has been rather limited, performative aspects of eating experiences have been particularly strong in chefs' interviews. We discussed how these can be framed as interaction trajectories $[13,14]$ using the concept of climax from dramatic composition [65]. More importantly, professional chefs' employed specific strategies for contrasting and balancing pleasurable and uncomfortable experiences for instance through intense tastes, or conflicting tastes and smells. These findings extend HCI ones on the value of orchestrating suspense and intrigue in gaming experiences [13], with fresh perspectives on how climax can be dramaturgically orchestrated through elicitation of positive as well as negative emotional responses. These open up novel design opportunities to design for surprise and climax in user experience and its trajectories.

\subsection{Design Space for HFI: Charts as Inspirational and Generative Design Tools for HFI Experiences}

We now turn our attention to how the design space for HFI [97]can be better articulated and further explored from the lens of eating experiences. By drawing on the key findings from the two studies and the above implications 
for HFI design research that they informed, we further focused on the sensory, emotional, communicative, and performative aspects of eating experiences. Since the temporal dimension has also emerged as important, cutting across each of these aspects, we have also focused in this section on temporal experiences.

An inspiring way to articulate the design space is through visualizations showing for instance significant points within the design space where the previously developed HFI technologies can be seen, and future ones yet to be designed, can be envisaged. For this, we drawn inspiration from Ashby diagrams [9] developed in material science. Originally, they were tools for simultaneously representing visually material qualities in order to support the selection of materials. These diagrams have been also used to illustrate aesthetic [11] and sensory [162] qualities of materials.

As mentioned above, the sensory, emotional, performative, communicative, and temporal aspects of eating experiences represent key themes emerging across the two studies, and therefore important in capturing the design space, and to represent through Ashby diagrams. We developed 6 charts which are further described. The axes of each chart were identified based on the key concepts relevant to its specific experience, such as emotional valence and arousal for emotional experiences [163]. In identifying the charts' axes, we also aimed to create two dimensional spaces within which to place the core of the findings from our two studies. For example, the identified types of stimuli from literature review were placed as design exemplars [169] and represented in the charts through oval shapes, to differentiate them from the interviews' findings represented through hexagonal shapes. For example in the first Chart, each shape, be it oval or hexagonal, represents a sensory experience with food that is color-coded for each specific sense. The latter was an important design decision in the construction of charts, illustrating the important role of the body within all the key aspects of eating experiences highlighted by the charts. These sensory aspects also ensured a useful cohesion across all charts, tied together through the important role of the body and bodily senses in each of these experiences. The oval shapes are also relatively sized, so that the larger their size, the greater the weighting of their content within the literature review's findings.

Our charts are intended as inspirational and generative design tools to support ideation and co-design of novel eating experiences, user experience with food, or even multisensory user experience. We do not claim that the six charts are exhaustive, as future work can better populate the charts we generated, or even lead to new charts. They offer however useful starting points for mapping the complex design space of HFI as they target empirically grounded, key aspects of eating experiences. Their generative quality is reflected in the novel opportunities for design that they can open up, especially in the areas of the charts limitedly covered by the existing findings, such as the limited interest in low arousal food experiences in the Emotional chart. In this respect, the charts further extend previous reviews [16] towards design knowledge for HFI and multisensory user experience more broadly. Similarly, there have been attempts to outline the range of experiential qualities of taste [139] or color [179], and whilst useful, most of previous work has been limited to unimodal experiences. In contrast, our charts integrate sensory fragments across all senses, both internal and external, alongside broader social and cultural ones, providing thus, a richer picture into food as a resource for design.

Whilst themes such as emotional, communicative and temporal experience have been the explicit focus of some HCI research $[21,55,56]$ what is presented here brings together literature and insights from our interviews to more fully appreciate the scope of emotional, communicative and temporal experience that can be designed for via food, alongside sensory and performative experiences which have been also less explored in the context of HFI. The charts are available for download from https://fooddesigncharts.com. Each chart is provided both without and with 
the experience fragments placed within their area. This allows designers to explore the provided axes without bias from previous work, as well as to engage with the design gaps within the previous work, and their opportunities.

\subsubsection{Senses Leveraged in Eating Experiences Chart}

The chart in Figure 3 highlights the important role of the body and bodily senses for eating experiences. It maps the sensory experiences with food across two axes: unimodal vs multimodal experiences, and internal vs external senses. As the chart illustrates, the reviewed, previous HFI work has explored mostly external, unimodal senses involved in eating experiences such as visual, audio, touch, touch, as well as taste or smell, with limited efforts focusing on internal senses, or multimodal experiences. Indeed, metabolization and digestion involve combinations of sensory information but their HFI exploration has been limited when compared to taste, visual and flavor aspects of eating experience.

The charts highlights how taste and smell are arguably more internal than audio-visual sensory aspects of experience, requiring contact with receptors within the body. Touch of mouth and hand sits between these two and together, form the group of unimodal sensory aspects of food. Flavor-based experiences are particularly interesting given their multimodal quality. Flavor-based experiences can involve merely external senses, especially based on electrically stimuli like in multisensory interfaces, but more importantly, they can also involve internal senses when based on the chemical stimuli of consumed food or drinks. This is an important outcome, indicating the value of food- and drinks-based flavor experiences excellently positioned to support rich multimodal experiences involving most or all of the senses. The chart also illustrates potential for innovative interfaces focused on the less explored internal senses.

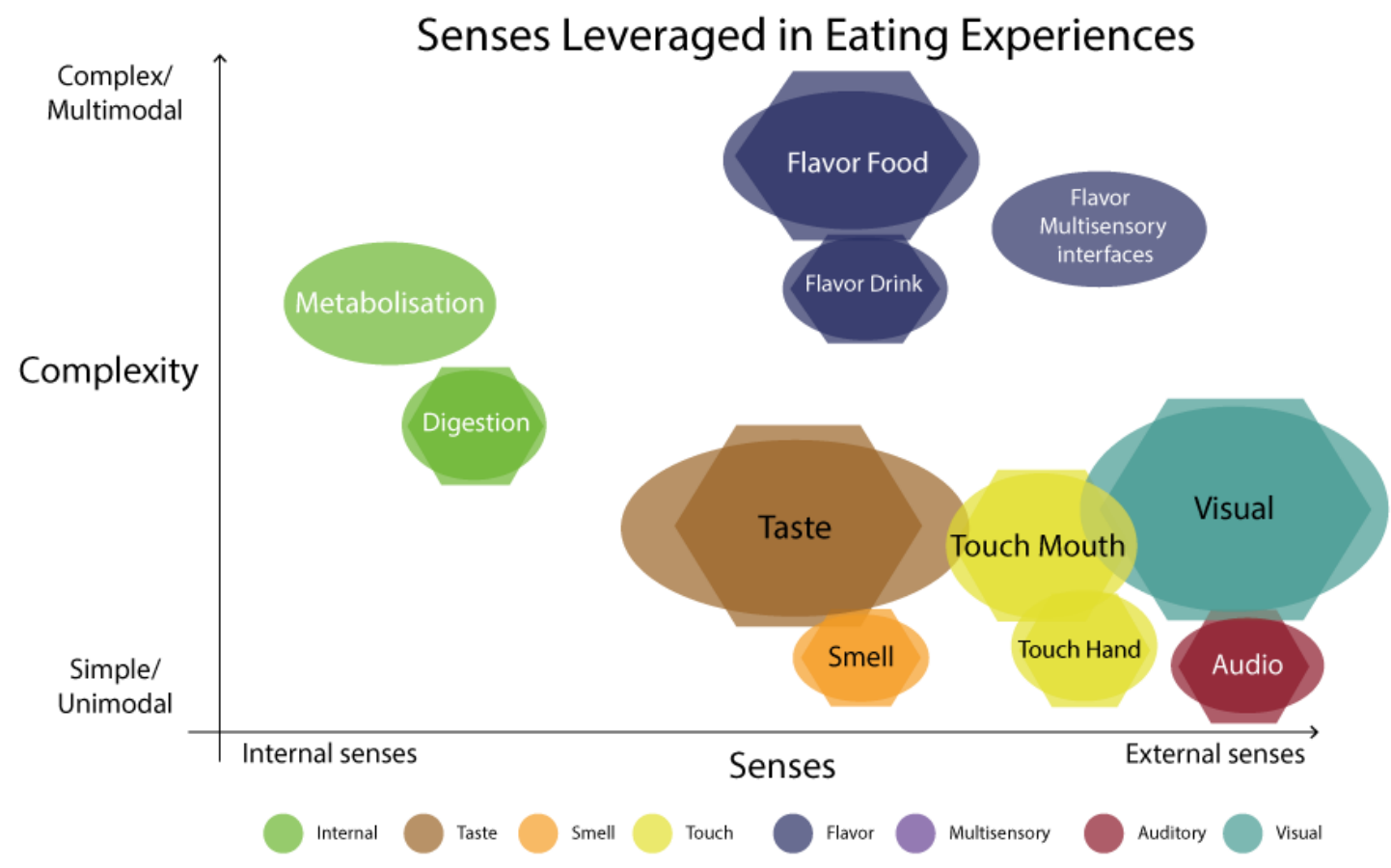

Figure 3 Senses leveraged in eating experiences chart 


\subsubsection{Sensory Stimulation/Deprivation Chart}

Given the importance of sensory aspects in eating experiences, we generated a second chart (Figure 4) using the same axes as in Figure 3 but placing within the different types of stimuli leveraged when designing for eating with, and around food. As the chart in Figure 4 illustrates, previous HFI work has explored mostly the external five senses, both separately like in unimodal experiences, and together like in multimodal experiences. Interestingly, all these stimuli have targeted primarily the sense of taste, with surprisingly less work focusing on smell. In contrast, professional chefs have shown specific interest in the sense of smell, not only for its stimulation but also deprivation.

Here, for example, we have seen chefs' effort to control smell through physically enclosing the odor in a serving bowl with a biscuit placed on top, to be released at the moment of eating. It is also worth noticing the limited focused on sensory deprivation in previous HFI work, although findings have shown that this is a valid pathway to stimulate the nondeprived senses [151,152]. The prototypical unimodal experience is electronic taste stimulation, although we have also seen chemical stimulation of taste through tastants, usually with at least another sensory stimulation such as colored lights, music, or texture for in mouth haptic experience. These taste base stimuli were intended to either augment or alter the taste sensation through additional sensory modalities.

Finally, most work in HFI has explored the multimodal experiences stimulating flavor through electronic stimuli or foodstuff, often with additional external stimuli. This involved combining food with visual or auditory stimuli to enhance the flavor experience. In the case of electronic taste stimuli, these were often combined with other chemical and non-chemical stimuli, including thermal, auditory, visual and olfactory. 


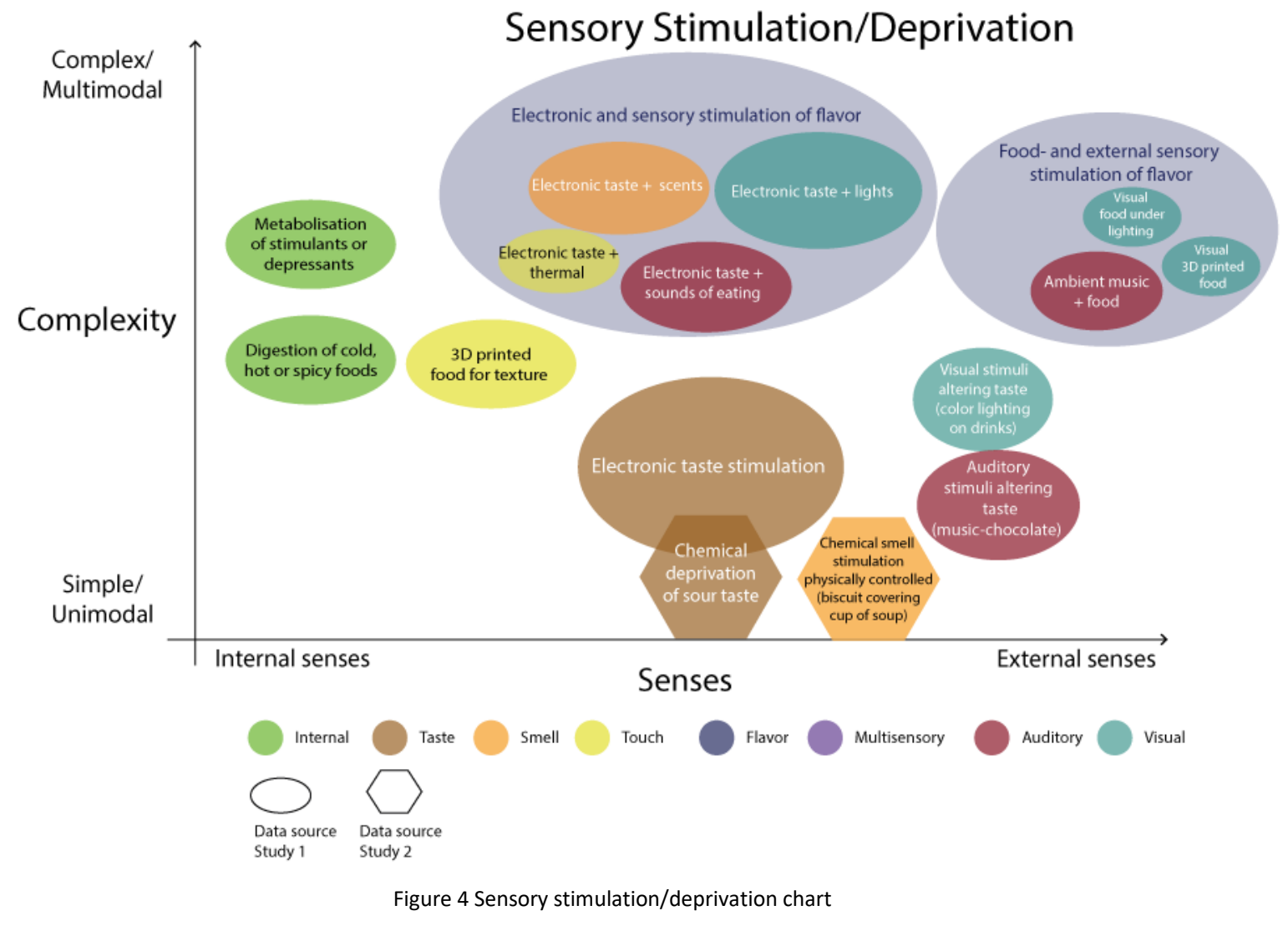

\subsubsection{Emotional Experiences}

The following chart (Figure 5) captures the emotional experiences related to eating, and in particular the relationship between tastes or flavors, on the one hand, and emotions, on the other hand. The chart draws from the HFI research on taste-emotion mapping [55,170,179], as well as chefs' accounts on how tastes and flavors are leveraged to elicit emotional responses, and therefore can be used to design for specific emotional experiences.

The chart is organized along the two axes of emotional valence and arousal informed by the Russell's circumplex model of affect [163]. As shown in Figure, 5 interview study indicates chefs' extensive focus on the value of tastes and flavors for eliciting emotions, usually of mid to high intensity. These outcomes considerably extend the previous HFI work in this space, where the focus has been mostly on the mapping of sweet/bitter tastes to positive/negative emotions, respectively, as seen in game applications or intimate communication systems. In contrast, chefs tend to explore the entire range of basic tastes, i.e., sweet, bitter, as well as salty, sour and umami, as well as specific flavors such as spiciness or culturally rich ones. 


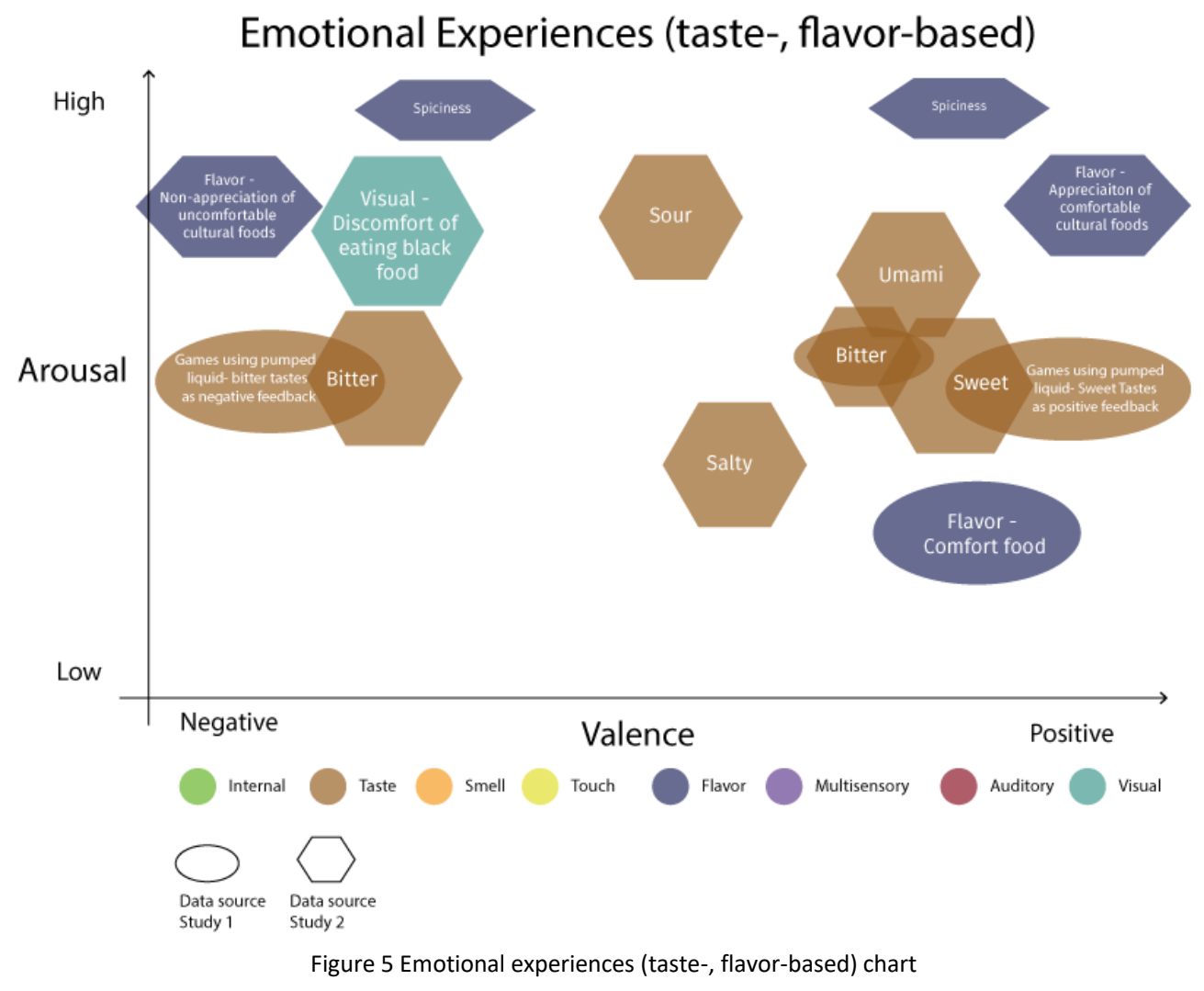

Within the space of mid to high emotional arousal, basic tastes cover the full range from positive to negative valence, with sour taste leading to the highest emotional arousal, salty to the lowest, umami (beside sweet) to positive emotions, while sour and salty leading to neither positive nor negative emotions, although sour tends to elicit more intense emotions. Interestingly, findings from Obrist and colleagues [126] suggested a similar hierarchy of intensity of taste experiences in decreasing order from sour, umami, bitter to sweet and salt, which our findings extend by showing that the same order also marks the intensity of emotional responses associated with these tastes.

Also interesting is that bitter taste is associated not only to negative but also positive emotions, extending previous findings in HFI showing only the unpleasantness of bitter taste [126]. It is possible that the positive emotions associated with bitter taste involve appreciation as the acquired taste for bitter food can develop over one's lifetime [55]. This opens up interesting avenues for dynamically evolving emotional responses to taste, over short or long time frames. Interview findings also indicate the cultural value of food for emotional experiences. Indeed, the (lack of) appreciation of cultural foods is associated with high intensity emotional experiences, with familiarity ensuring positive emotions and non-familiarity ensuring negative ones. The specific value of personal or culturally meaningful foodstuff for eliciting emotions opens up novel design opportunities.

Unfamiliarity can also be entailed by using black as the color of food to create tension between the food's lack of visual appeal and its pleasant taste. Interestingly, such tensions derived from clashes of information from different modalities, the appreciation or not for culturally specific flavors, or spiciness as flavor, also tend to elicit the highest intensity of emotional experiences, both positive and negative. 
The importance of tastes' familiarity for positive emotions and unfamiliarity for ambivalent ones, i.e., surprise or suspicion has been previously suggested [126]. Our findings extend them to flavors and their cultural appreciation or lack of. While not directly supported by our findings, the potential of umami taste to be either loved or hated [126], may be subtly linked to its cultural appreciation, where we have indeed seen that umami was one of the dominant taste like in the example of rotten fish. Our findings also suggest the value of designing for surprise through such unexpected clashes between foodstuff's color [179] and taste that can lead to positive and negative experiences by shaping the anticipation of, and the actual flavor. They also suggest the value of understanding users' preference for culturally specific flavors, i.e., spiciness, in order to elicit high intensity emotions.

Interestingly, most of the findings indicate a bias towards positive emotional responses entailed by eating [36], with less focus on negative ones. As suggested by work on emotions and eating [36], there remains the question of how people may wish to engage with food they know, or believe, will elicit negative experiences. As discussed, with the acquired taste for bitter, there is potential for rich experiences to evolve over time, changing one's relationship with a particular food. Alternatively, negative food experiences may provide a way of designing tools for reflection or catharsis through supporting re-experiencing and re-embodying negative emotions as part of processing them [115]. Not at least, a well-documented finding in food research, also illustrated in the chart is the low arousal positive emotions associated with comfort food [55,186]. Moreover, emotional experiences can be not only expressed through food [56] but also regulated through food, helping people to cheer up or calm down [56]. The value of food for emotion regulation has just started to be explored [60] and can open up exciting avenues for future design, especially in the domain of healthy eating.

\subsubsection{Communicative Experiences}

The chart in Figure 6 explores food as a medium for communication. The entailed communicative experiences are organized along form of information, ranging from explicit or factual data to implicit or more abstract data, and the social dimension of information ranging from personally to communally relevant. Most of the previous work in HFI has focused on personally relevant information, both explicit and implicit. Personally relevant explicit information predominantly involved data edibilization for communicating factual data from tracked fitness data to CV information. Such data has been in the form or personalized flavor or the visual form of messages projected or printed on food surfaces [103]. For example, Edipulse [92] provides messages printed in chocolate as rewards for reaching exercise goals. Also explicit are messages communicated through food with the aim of supporting remote connectiveness, often in intimate relationship. So the social dimension of such communicative experiences extends from personally to dyadically relevant. 


\section{Communicative Experiences}

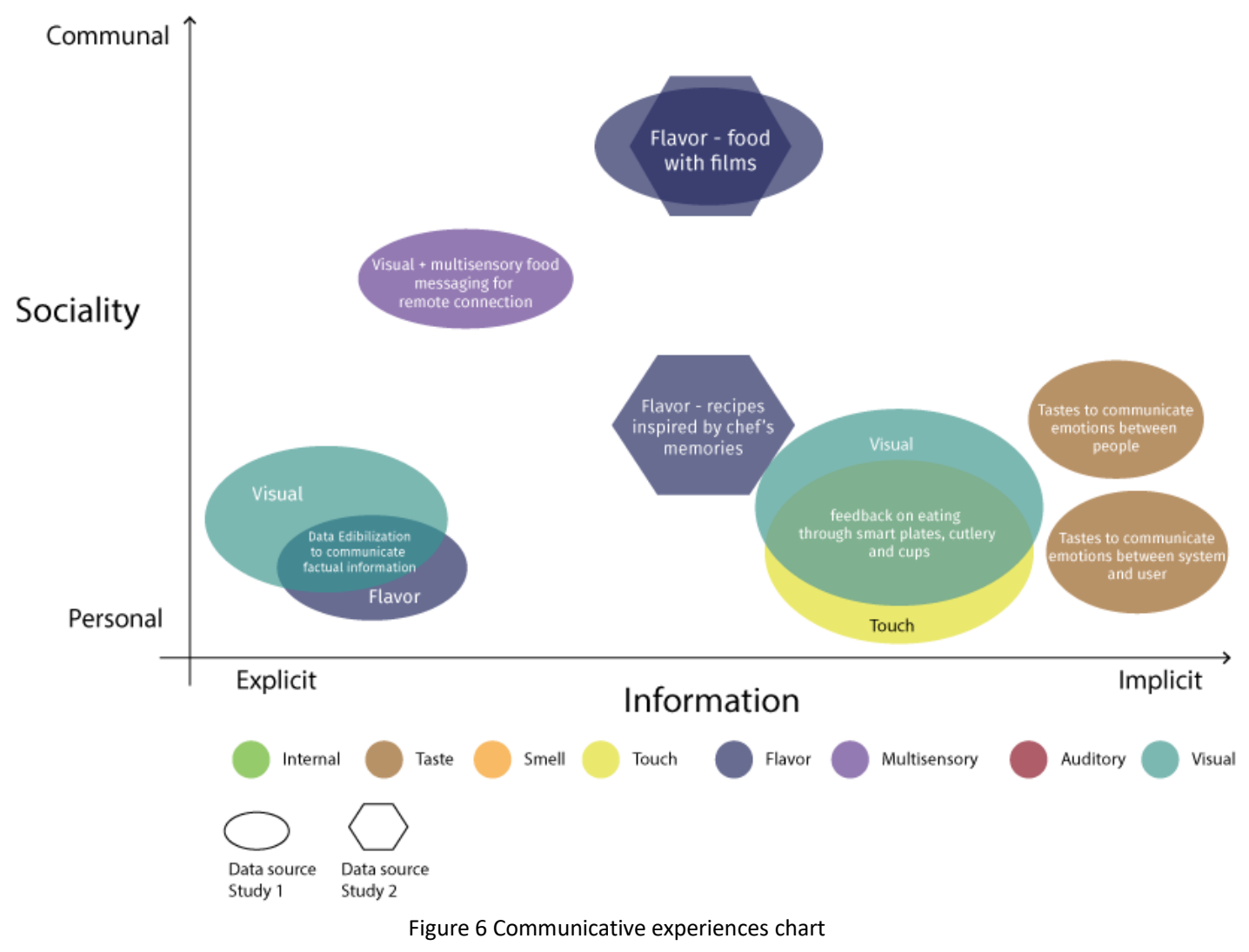

In contrast to the explicit information content, at the other end of the $\mathrm{x}$ axis, we have implicit one, such as tastes communicating emotions, either during one's interaction with a system, or between two people. Such affective communication can be supported through personalized flavors, exchanged for instance among partners in romantic relationships [57]. Such content takes the form of foodstuff itself and its tastes being used to communicate emotions, that differs from the more explicit messages being written on food. In the context of a romantic relationship, if one partner offers food to express their feelings, the food itself being consumed by their partner is symbolic, expressing an appreciation for that feeling. Implicit content can also take the form of smart tableware used to communicate and help regulate aspects such as the speed or amount of food being eaten, in order to support healthier eating behaviors. These can be used both by individual users or between remote co-diners in order to support them to synchronize their dining experiences. The chart's elements described above are based on findings from previous HFI research. Interestingly, the findings from chefs' interviews focus on the use of flavors for communicating narratives to diners usually in a restaurant, as a larger audience with limited social ties among themselves. The narratives being communicated reflect flavor-memory connections, and take the form of flavor-based memories that inspired the chefs to create those dishes. The social dimension of restaurant dining can be increased when the audience is further immersed not only in sharing meals, but for instance the experience of watching a film together. 
Although less common, this is an example of communal communicative eating experiences. These less explored communal commutative experiences open up an interesting design space where food and shared interactions can be designed for. For instance, one can imagine smart TV shows and tailored meals with the aim of augmenting the communal experiences of one's social network of remote family and friends.

\subsubsection{Performative Experiences}

Figure 7 shows the chart describing performative experiences associated with eating food. Performative experiences have beginning, middle and end. Duration as temporal dimension can range from short such as the time needed to take a mouthful, to long such as the time needed to consume a multi-course meal. Thus, the chart opens up opportunities to design for longer experiences that extend beyond typical 'short-term' food experiences [44].

\section{Performative Experiences}

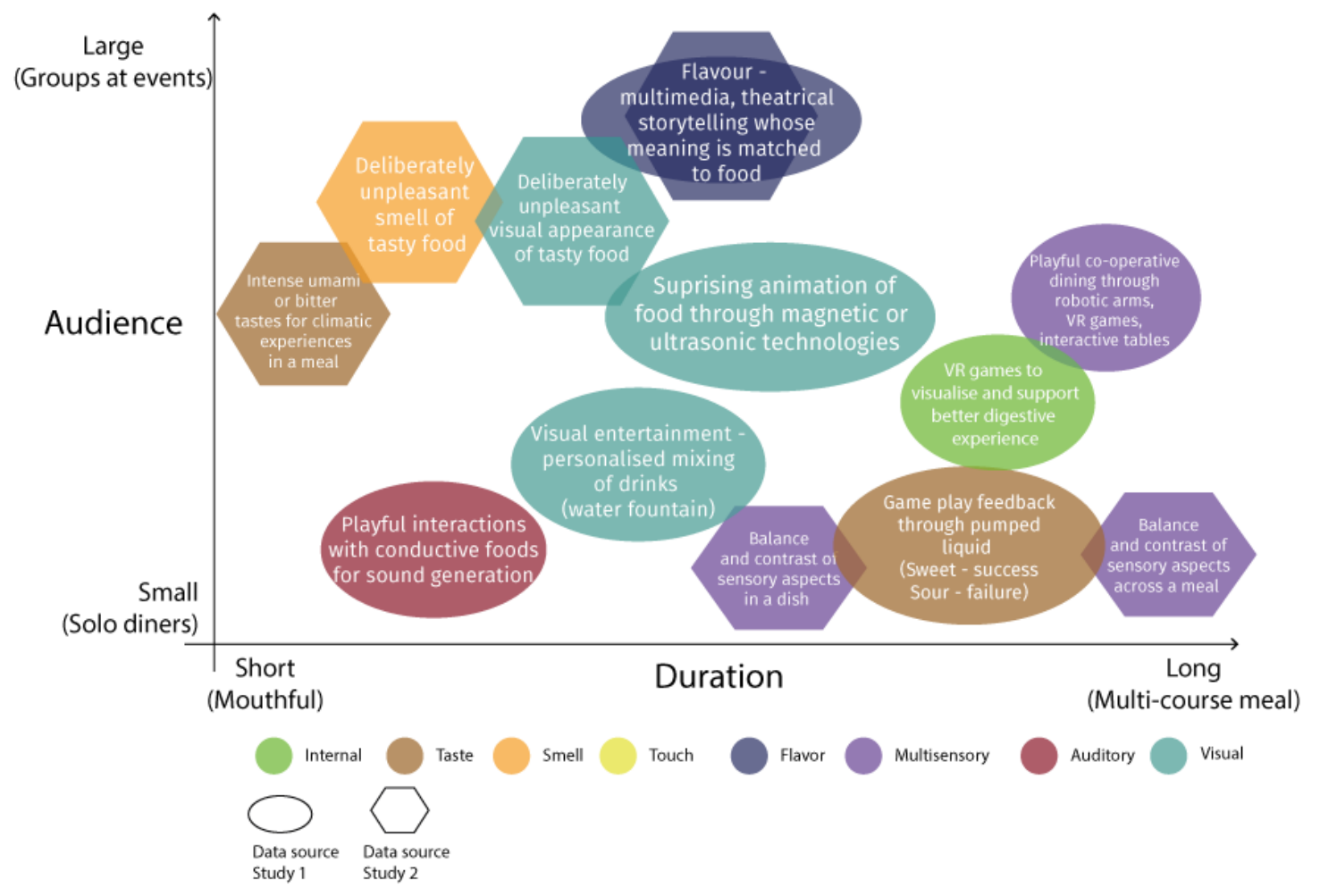

Figure 7 Performative experiences chart

Performative experiences also have audiences, ranging from solo diners or two co-diners to larger groups. The main aspects of performative experiences include playfulness, storytelling, surprise, climax, tension and balance. While the former three aspects have been covered by previous HFI research, the last four aspects have emerged as important from chefs' interviews. In addition, while most of the work in HFI has focused on shorter performative experiences, the longer ones have received somehow less attention, especially for larger groups. 
Playfulness is an aspect of performative experiences that can be of short or longer duration, involving either solo diners or more often two co-diners. It is supported when the consumption of foods generates playful outputs in a range of modalities through sound, visual, robotic and VR interfaces. Playfulness also relates to gamification principles of positive/negative feedback through sweet/sour tastes. Storytelling aspect of performative experiences involves integration of flavors with a multimedia based story, usually for larger audiences.

For instance, StreetSauce [42] supports food sharing to encourage the sharing of personal stories among marginalized women. There is also potential to further learn from (religious) rituals which use food as parts of historical retelling (e.g. unleavened bread in the Jewish faith) to support bodily connection to their narratives through human food interaction. Not at least, surprise has been supported in previous HFI through unexpected, almost "magic" animation of foodstuff through invisible technologies such as magnetic or ultrasonic-based ones [183]. The ability for food to become responsive to users' interaction is particularly intriguing. We can imagine for instance tuning a guitar while foodstuff is transforming according to the frequency of the string being plucked. It could begin as an out of tune, spiky blob and slowly smooth and round to a perfect sphere which can be picked up and eaten alongside the exact 'sweet' tone.

From chefs' interviews, the surprise aspect of performative experiences is usually ensured alongside tension, such as in the case of deliberately unpleasant appearance or smell of tasty food. Usually such surprising or tension aspects relate to a short, mouthful experiences. Climactic aspects of performative experiences leverage taste, usually as intense umami or bitter, which makes them particularly memorable. For supporting such climax, the sensory aspects of tension and balance are key, both within dishes, and across multi-course meals. For instance, a 'palate cleanser' such as citrus taste may be used to cut through umami, or cold foodstuff to counter a hot mouth.

\subsubsection{Temporal Experiences}

Like all experiences, eating ones unfold in time, but they also undergo unique chemical transformations from the eating of food to its metabolization. The chart for temporal experiences in Figure 8 is organized along the duration of eating experiences from short ones lasting seconds such as those involving tastants, to long ones lasting up to eight hour such as those involved in metabolization of food. The vertical axis allows for a broader view of eating experiences, extending to both before, and after, the moment of eating, i.e., including chewing and swallowing. The chart in Figure 8 shows that most of previous HFI research, and findings from chefs' interviews have focused most often on the moment of eating and its key temporal aspects such as ephemerality, lingering, and eating speed. In this respect, the mere act of eating food is an ephemeral [44], short lasting experience. Tastes stimuli support shorter experiences, during and just after the moment of eating which Obrist and colleagues called synchronic [126]. In addition, for specific intense tastes or flavors, such as spiciness, these ephemeral sensations become extended through the trigeminal stimulation to slightly longer, lingering experiences.

Flavors may also linger when their material properties are characterized by viscous liquids or stickier solids that stay longer in the mouth as they require more saliva before swallowing. These findings extend the temporal quality of taste to linger beyond the initial stimulation which Obrist and colleagues called diachronic [126] to flavor experiences. Moreover, the experience of the same taste can change over time through repeated exposure, as people gain appreciation for "acquired tastes", further extending the diachronic structure over the life span. Such findings open up opportunities for designing novel, more embodied taste-based representations of temporal metadata such as recentness of an update, or duration of a video file. 


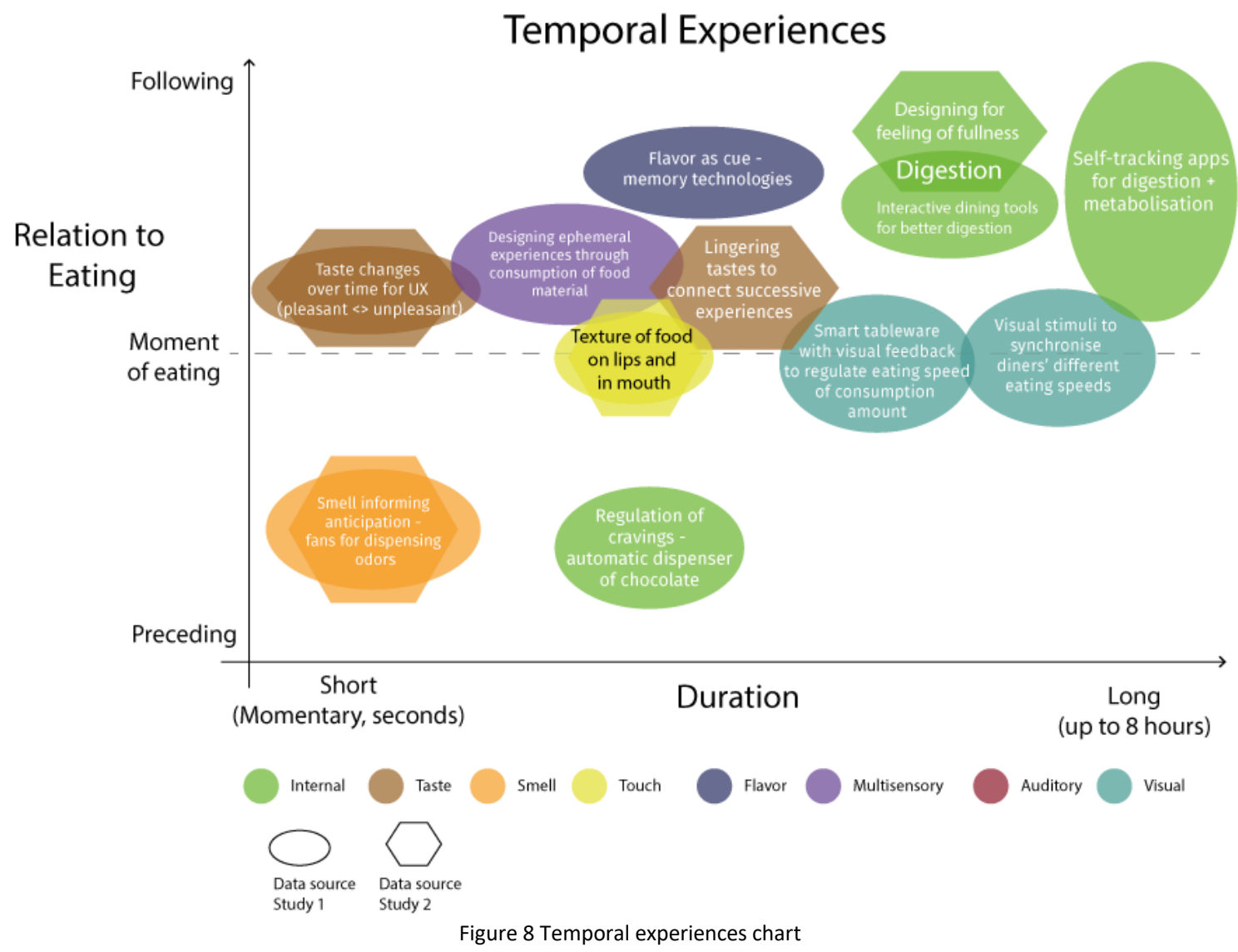

With respect to eating speed, visual stimuli or smart tableware can be used to increase awareness of the speed in order to help diners to slow down the speed for more chowing and ultimately healthier eating, or to allow codiners to synchronize their eating speeds, i.e., KIZUNA system [136], or the balancing table [122]. Such systems open up interesting opportunities to leverage social dimension in order to design for the temporal aspects of food experiences. A food experience can be made faster through a dining partner eating quickly, and vice versa for a slower experience. Little explored so far has been the potential for avatars to curate food experiences, and particularly slower ones such as those involved in mindful eating.

In contrast to the sense of taste primarily stimulated during eating, following the moment of eating, the internal senses of digestion and metabolization take precedence. Most of the previous HFI work in this space has focused on supporting better digestion and metabolization for healthy eating through interactive dining tools such as VR based games [7,8]. Digestion in particular has also been explored in chefs' practice through creating dishes aimed to support the feeling of fulness. A growing body of HFI work has focused on longer experiences associated with digestion and metabolization through self-tracking apps of how the body responds to specific foodstuff, that echoes the emergence of citizen scientists hacking their diet $[40,41]$. Such connection between the future self and the present eating behavior reflects the assumption 'you are what you eat'. Self-tracking apps approach food almost like drugs in that the moment of consumption tends to be ignored and the priority is placed on the impact of food 
once it is digested. Promoting healthy eating can often rely on deferring immediate gratification for future healthier self, so that this tension between the present experience and future one requires careful design consideration. Ethical concerns regarding such apps have also emerged especially when they support problematic use of 'thinspiration' images or consumption of caffeinated products to counter tiredness. Addressing such concerns requires much sensitivity from designers to limit harm and better support informed decisions for healthy future experiences. An interesting design opportunity for such apps, is to extend their focus on the moment of eating, for instance through digital implementation of mindful eating interventions.

Another interesting illustration of experiences following the moment of eating are flavors that can cue specific autobiographical memories. In this way, foodstuff as memory cues provide an experience after the moment of eating, but also connect to previous food experiences, creating associations between repeated experiences over time. For instance, recent such work involved personalized 3D printed food [57] used to support reminiscing in old age [58], that also builds on the growing HCI work on memory technologies.

While most of the work highlighted in this chart focuses on or after the moment of eating, surprisingly less work has focused on the time prior to eating. Short experiences in this respect involve seconds prior to eating, for instance with the pleasant smells being provided in order to heighten the anticipation of taste. Particularly interesting is the focus on self-regulation of craving for unhealthy foods such as sweet treats, through automatic dispensing technologies intended to train the ability to resist temptation and delay gratification. Future HFI research focusing on this gaps can open up new design opportunities where temporal dimension of eating experiences can be deliberately addressed. This can draw from the growing HCI interest in the topic of time [145], which has limitedly been explored in the context of HFI.

\section{CONCLUSION}

We conducted a systematic literature review of 109 papers, and interviews with 18 professional chefs in order to explore and articulate the opportunities of designing for eating experiences. Findings identified the sensory, emotional, cognitive and social aspects of eating experiences explored in HFI research, the diverse technologies supporting these experiences and the important value of the human body in these eating practices. Interviews with professional chefs shown the value of considering the relation between flavors and emotion, and the importance of sensory stimulation and deprivation for pleasurable eating experiences as well as the uncomfortable ones, and of cultural aspects for appreciating both. We also presented a conceptual framework of designing for user experience that draws from frameworks of designing for taste experiences and for user experience, with elements of dramaturgical composition, while advancing the mouth and the gut as new sites for HFI.

These findings led to five implications for HFI design research on the value of multisensory flavor experiences, external and internal sensory stimulation and deprivation, flavor-emotion mapping, social and cultural aspects of eating to communicate meaning, and designing for dramaturgical climax. Finally, we also proposed six charts as novel inspirational and generative design tools for HFI experiences focused on senses, sensory stimulation, as well as emotional, communicative, performative and temporal experiences. 


\section{ACKNOWLEDGMENTS}

This work was supported by the Designing Multisensory Interactions: Emotional User Experience of 3D PrintedFood Consumption project (Grant Number 1962364) funded by EPSRC and Dovetailed Ltd. under a CASE PhD award and AffecTech: Personal Technologies for Affective Health, Innovative Training Network funded by the H2020 People Programme under Marie Skłodowska-Curie GA No 722022.

\section{REFERENCES}

References marked with an asterisk indicate studies included in the systematic review.

*[1] Nur Ellyza Abd Rahman, Azhri Azhar, Mohammad Abdullah Mohamad Johar, Kasun Karunanayaka, Adrian David Cheok, Jade Gross, and Andoni Luis. 2016. Magnetic Dining Table and Magnetic Foods. In Proceedings of the 13th International Conference on Advances in Computer Entertainment Technology (ACE '16), Association for Computing Machinery, New York, NY, USA, 1-6. DOI:https://doi.org/10.1145/3001773.3001809

*[2] Nur Ellyza Abd Rahman, Azhri Azhar, Kasun Karunanayaka, Adrian David Cheok, Mohammad Abdullah Mohamad Johar, Jade Gross, and Andoni Luis Aduriz. 2016. Magnetic dining table interface and magnetic foods for new human food interactions. In Proceedings of the fourth international conference on human agent interaction (HAI '16), Association for Computing Machinery, New York, NY, USA, 79-81. DOI:https://doi.org/10.1145/2974804.2980504

*[3] Dilrukshi Abeyrathne, Roshan Lalintha Peiris, Nimesha Ranasinghe, Owen Noel Newton Fernando, and Adrian David Cheok. 2010. Food internet communication. In Proceedings of the 7th international conference on advances in computer entertainment technology (ACE '10), Association for Computing Machinery, New York, NY, USA, 49-52. DOI:https://doi.org/10.1145/1971630.1971645

[4] Miquel Alfaras, Vasiliki Tsaknaki, Pedro Sanches, Charles Windlin, Muhammad Umair, Corina Sas, and Kristina Höök. 2020. From Biodata to Somadata. In Proceedings of the 2020 CHI Conference on Human Factors in Computing Systems (CHI '20), Association for Computing Machinery, New York, NY, USA, 1-14. DOI:https://doi.org/10.1145/3313831.3376684

[5] Ferran Altarriba Bertran, Jared Duval, Katherine Isbister, Danielle Wilde, Elena Márquez Segura, Oscar Garcia Pañella, and Laia Badal León. 2019. Chasing Play Potentials in Food Culture to Inspire Technology Design. In Extended Abstracts of the Annual Symposium on Computer-Human Interaction in Play Companion Extended Abstracts (CHI PLAY '19 Extended Abstracts), Association for Computing Machinery, Barcelona, Spain, 829-834. DOI:https://doi.org/10.1145/3341215.3349586

[6] Ferran Altarriba Bertran, Samvid Jhaveri, Rosa Lutz, Katherine Isbister, and Danielle Wilde. 2019. Making sense of human-food interaction. In Proceedings of the 2019 CHI conference on human factors in computing systems (CHI '19), Association for Computing Machinery, New York, NY, USA, 1-13. DOI:https://doi.org/10.1145/3290605.3300908

*[7] Peter Arnold, Rohit Ashok Khot, and Florian "Floyd" Mueller. 2018. "You Better Eat to Survive": Exploring cooperative eating in virtual reality games. In Proceedings of the twelfth international conference on tangible, embedded, and embodied interaction (TEI '18), Association for Computing Machinery, New York, NY, USA, 398-408. DOI:https://doi.org/10.1145/3173225.3173238

*[8] Eshita Sri Arza, Harshitha Kurra, Rohit Ashok Khot, and Florian "Floyd" Mueller. 2018. Feed the food monsters! Helping co-diners chew their food better with augmented reality. In Proceedings of the 2018 annual symposium on computer-human interaction in play companion extended abstracts (CHI PLAY '18 extended abstracts), Association for Computing Machinery, New York, NY, USA, 391-397. DOI:https://doi.org/10.1145/3270316.3271520

[9] M Ashby and D Cebon. Materials selection in mechanical design. 10.

[10] Malika Auvray and Charles Spence. 2008. The multisensory perception of flavor. Conscious Cogn 17, 3 (September 2008), 1016-1031. DOI:https://doi.org/10.1016/j.concog.2007.06.005

[11] Camilo Ayala-Garcia and Valentina Rognoli. 2017. The New Aesthetic of DIY-Materials. The Design Journal 20, sup1 (July 2017), S375-S389. DOI:https://doi.org/10.1080/14606925.2017.1352905 
[12] L. M. Bartoshuk. 1978. The psychophysics of taste. Am J Clin Nutr 31, 6 (June 1978), 1068-1077. DOI:https://doi.org/10.1093/ajcn/31.6.1068

[13] Steve Benford, Andy Crabtree, Stuart Reeves, Jennifer Sheridan, Alan Dix, Martin Flintham, and Adam Drozd. 2006. The Frame of the Game: Blurring the Boundary between Fiction and Reality in Mobile Experiences. In Proceedings of the SIGCHI Conference on Human Factors in Computing Systems (CHI '06), Association for Computing Machinery, New York, NY, USA, 427-436. DOI:https://doi.org/10.1145/1124772.1124836

[14] Steve Benford, Gabriella Giannachi, Boriana Koleva, and Tom Rodden. 2009. From Interaction to Trajectories: Designing Coherent Journeys Through User Experiences. In Proceedings of the SIGCHI Conference on Human Factors in Computing Systems (CHI '09), ACM, New York, NY, USA, 709-718. DOI:https://doi.org/10.1145/1518701.1518812

[15] Steve Benford, Chris Greenhalgh, Gabriella Giannachi, Brendan Walker, Joe Marshall, and Tom Rodden. 2013. Uncomfortable User Experience. Commun. ACM 56, 9 (September 2013), 66-73. DOI:https://doi.org/10.1145/2500468.2500889

[16] Ferran Altarriba Bertran, Katherine Isbister, Samvid Jhaveri, Danielle Wilde, and Rosa Lutz. 2018. Visualising the landscape of Human- Food Interaction research. Hong Kong (2018), 6.

*[17] Felix Brandmueller and Zhuying Li. 2017. Guts game: A game using ingestible sensors. In Extended abstracts publication of the annual symposium on computer-human interaction in play (CHI PLAY '17 extended abstracts), Association for Computing Machinery, New York, NY, USA, 625-631. DOI:https://doi.org/10.1145/3130859.3130866

[18] Wender L. P. Bredie, Hui Shan Grace Tan, and Karin Wendin. 2014. A Comparative Study on Facially Expressed Emotions in Response to Basic Tastes. Chem. Percept. 7, 1 (March 2014), 1-9. DOI:https://doi.org/10.1007/s12078-014-9163-6

*[19] Manu J. Brueggemann, Vanessa Thomas, and Ding Wang. 2018. Lickable cities: Lick everything in sight and on site. In Extended abstracts of the 2018 CHI conference on human factors in computing systems (CHI EA '18), Association for Computing Machinery, New York, NY, USA, 1-10. DOI:https://doi.org/10.1145/3170427.3188399

*[20] Merijn Bruijnes, Gijs Huisman, and Dirk Heylen. 2016. Tasty tech: human-food interaction and multimodal interfaces. In Proceedings of the 1st Workshop on Multi-sensorial Approaches to Human-Food Interaction MHFI '16, ACM Press, Tokyo, Japan, 1-6. DOI:https://doi.org/10.1145/3007577.3007581

*[21] Merijn Bruijnes, Gijs Huisman, and Dirk Heylen. 2016. Tasty Tech: Human-food Interaction and Multimodal Interfaces. In Proceedings of the 1st Workshop on Multi-sensorial Approaches to Human-Food Interaction (MHFI '16), ACM, New York, NY, USA, 4:1-4:6. DOI:https://doi.org/10.1145/3007577.3007581

[22] Leah Buechley and Hannah Perner-Wilson. 2012. Crafting technology: Reimagining the processes, materials, and cultures of electronics. ACM Trans. Comput.-Hum. Interact. 19, 3 (October 2012), 21:121:21. DOI:https://doi.org/10.1145/2362364.2362369

[23] Katie Bunnell. 2004. Craft and digital technology. Metsovo, Greece, 20. Retrieved from http://repository.falmouth.ac.uk/id/eprint/537

*[24] Felipe Reinoso Carvalho, Kris Steenhaut, Raymond van Ee, Abdellah Touhafi, and Carlos Velasco. 2016. Sound-enhanced Gustatory Experiences and Technology. In Proceedings of the 1st Workshop on Multisensorial Approaches to Human-Food Interaction (MHFI '16), ACM, New York, NY, USA, 5:1-5:8. DOI:https://doi.org/10.1145/3007577.3007580

[25] Kai Qin Chan, Eddie M. W. Tong, Deborah H. Tan, and Alethea H. Q. Koh. 2013. What do love and jealousy taste like? Emotion 13, 6 (December 2013), 1142-1149. DOI:https://doi.org/10.1037/a0033758

[26] Bin-Bin Chen and Lei Chang. 2012. Bitter struggle for survival: Evolved bitterness embodiment of survival motivation. Journal of Experimental Social Psychology 48, 2 (March 2012), 579-582. DOI:https://doi.org/10.1016/j.jesp.2011.11.005

*[27] Ying-Yu Chen, Kelda Baljon, Bonnie Tran, Daniela K. Rosner, and Alexis Hiniker. 2018. The stamp plate and the kicking chair: playful productivity for mealtime in preschools. In Proceedings of the 17th ACM Conference on Interaction Design and Children (IDC '18), Association for Computing Machinery, New York, NY, USA, 373-380. DOI:https://doi.org/10.1145/3202185.3202759 
*[28] Ying-Yu Chen, Jason Yip, Daniela Rosner, and Alexis Hiniker. 2019. Lights, music, stamps! Evaluating mealtime tangibles for preschoolers. In Proceedings of the thirteenth international conference on tangible, embedded, and embodied interaction (TEI '19), Association for Computing Machinery, New York, NY, USA, 127-134. DOI:https://doi.org/10.1145/3294109.3295645

*[29] Foong-Yi Chia and Daniel Saakes. 2014. Interactive training chopsticks to improve fine motor skills. In Proceedings of the 11th conference on advances in computer entertainment technology (ACE '14), Association for Computing Machinery, New York, NY, USA. DOI:https://doi.org/10.1145/2663806.2663816

[30] Yoram Chisik, Patricia Pons, and Javier Jaen. 2018. Gastronomy meets ludology: Towards a definition of what it means to play with your (digital) food. In Proceedings of the 2018 annual symposium on computerhuman interaction in play companion extended abstracts, Association for Computing Machinery, New York, NY, USA, 155-168. Retrieved from https://doi.org/10.1145/3270316.3272056

[31] Jaz Hee-jeong Choi, Marcus Foth, and Greg Hearn (Eds.). 2014. Eat, Cook, Grow: Mixing Human-Computer Interactions with Human-Food Interactions. The MIT Press, Cambridge, Mass. Retrieved November 2, 2016 from http://lib.myilibrary.com?id=586295

[32] Rob Comber, Polly Barden, Nick Bryan-Kims, and Patrick Olivier. 2014. Not Sharing Sushi: Exploring Social Presence and Connectedness at the Telematic Dinner Party. In Eat, Cook, Grow: Mixing HumanComputer Interactions with Human-Food Interactions, Jaz Hee-Jeong Choi, Marcus Foth and Greg Hearn (eds.). The MIT Press, Cambridge, Mass., 65-80.

[33] Claudia Dauden Roquet and Corina Sas. 2021. Interoceptive Interaction: An Embodied Metaphor Inspired Approach to Designing for Meditation. In In Proceedings of the 2021 CHI Conference on Human Factors in Computing Systems (Yokohama, Japan) (CHI '21)., Association for Computing Machinery, New York, NY, USA.

[34] Michel Delville. 2007. Food, Poetry, and the Aesthetics of Consumption: Eating the Avant-Garde. Routledge, New York. DOI:https://doi.org/10.4324/9780203937792

[35] Jeannine F. Delwiche. 2012. You eat with your eyes first. Physiology \& Behavior 107, 4 (November 2012), 502-504. DOI:https://doi.org/10.1016/j.physbeh.2012.07.007

[36] Pieter M. A. Desmet and Hendrik N. J. Schifferstein. 2008. Sources of positive and negative emotions in food experience. Appetite 50, 2-3 (March 2008), 290-301.

DOI:https://doi.org/10.1016/j.appet.2007.08.003

[37] Garmt Dijksterhuis, Claire Boucon, and Elodie Le Berre. 2014. Increasing saltiness perception through perceptual constancy created by expectation. Food Quality and Preference 34, (June 2014), 24-28. DOI:https://doi.org/10.1016/j.foodqual.2013.12.003

*[38] Marketa Dolejšová. 2016. Deciphering a meal through open source standards: Soylent and the rise of diet hackers. In Proceedings of the 2016 CHI conference extended abstracts on human factors in computing systems (CHI EA '16), Association for Computing Machinery, New York, NY, USA, 436-448. DOI:https://doi.org/10.1145/2851581.2892586

[39] Markéta Dolejšová. 2018. Parlour of food futures: Future food forecasting as tarot prophecies. In Proceedings of the 30th australian conference on computer-human interaction (OzCHI '18), Association for Computing Machinery, New York, NY, USA, 593-597. DOI:https://doi.org/10.1145/3292147.3292226

*[40] Markéta Dolejšová and Denisa Kera. 2016. Fermentation GutHub: Designing for food sustainability in singapore. In Proceedings of the 2nd international conference in HCI and UX indonesia 2016 (CHIuXiD '16), Association for Computing Machinery, New York, NY, USA, 69-76. DOI:https://doi.org/10.1145/2898459.2898470

*[41] Markéta Dolejšová and Denisa Kera. 2017. Soylent diet self-experimentation: Design challenges in extreme citizen science projects. In Proceedings of the 2017 ACM conference on computer supported cooperative work and social computing (CSCW '17), Association for Computing Machinery, New York, NY, USA, 2112-2123. DOI:https://doi.org/10.1145/2998181.2998365

*[42] Markéta Dolejšová and Tereza Lišková. 2015. StreetSauce: Taste interaction and empathy with homeless people. In Proceedings of the 33rd annual ACM conference extended abstracts on human factors in computing systems (CHI EA '15), Association for Computing Machinery, New York, NY, USA, 1247-1252. DOI:https://doi.org/10.1145/2702613.2732777 
[43] Tanja Döring. 2016. The Interaction Material Profile: Understanding and Inspiring How Physical Materials Shape Interaction. In Proceedings of the 2016 CHI Conference Extended Abstracts on Human Factors in Computing Systems, ACM, San Jose California USA, 2446-2453. DOI:https://doi.org/10.1145/2851581.2892516

*[44] Tanja Döring, Axel Sylvester, and Albrecht Schmidt. 2013. A Design Space for Ephemeral User Interfaces. In Proceedings of the 7th International Conference on Tangible, Embedded and Embodied Interaction (TEI '13), ACM, New York, NY, USA, 75-82. DOI:https://doi.org/10.1145/2460625.2460637

[45] Elizabeth V. Eikey and Madhu C. Reddy. 2017. "It's Definitely Been a Journey": A qualitative study on how women with eating disorders use weight loss apps. In Proceedings of the 2017 CHI conference on human factors in computing systems (CHI '17), Association for Computing Machinery, New York, NY, USA, 642654. DOI:https://doi.org/10.1145/3025453.3025591

[46] N Elias. 1978. The civilising process. Vol I the history of manners. Vol II state formation and civilization. Basil Blackwell., Oxford.

[47] Geremy Farr-Wharton, Marcus Foth, and Jaz Hee-jeong Choi. 2013. EatChaFood: Challenging technology design to slice food waste production. In Proceedings of the 2013 ACM conference on pervasive and ubiquitous computing adjunct publication (UbiComp '13 adjunct), Association for Computing Machinery, New York, NY, USA, 559-562. DOI:https://doi.org/10.1145/2494091.2497311

[48] Hasan Shahid Ferdous, Bernd Ploderer, Hilary Davis, Frank Vetere, and Kenton O'hara. 2016. Commensality and the Social Use of Technology during Family Mealtime. ACM Trans. Comput.-Hum. Interact. 23, 6 (December 2016), 1-26. DOI:https://doi.org/10.1145/2994146

[49] Hasan Shahid Ferdous, Bernd Ploderer, Hilary Davis, Frank Vetere, Kenton O'Hara, Geremy Farr-Wharton, and Rob Comber. 2016. TableTalk: Integrating Personal Devices and Content for Commensal Experiences at the Family Dinner Table. In Proceedings of the 2016 ACM International Joint Conference on Pervasive and Ubiquitous Computing (UbiComp '16), ACM, New York, NY, USA, 132-143.

DOI:https://doi.org/10.1145/2971648.2971715

[50] Jennifer Fereday and Eimear Muir-Cochrane. 2006. Demonstrating Rigor Using Thematic Analysis: A Hybrid Approach of Inductive and Deductive Coding and Theme Development. International Journal of Qualitative Methods 5, 1 (March 2006), 80-92. DOI:https://doi.org/10.1177/160940690600500107

[51] Jodi Forlizzi and Katja Battarbee. 2004. Understanding Experience in Interactive Systems. In Proceedings of the 5th Conference on Designing Interactive Systems: Processes, Practices, Methods, and Techniques (DIS '04), ACM, New York, NY, USA, 261-268. DOI:https://doi.org/10.1145/1013115.1013152

*[52] Ayelet GalOz, Orad Weisberg, Tal KerenCapelovitch, Yair Uziel, Ronit Slyper, Patrice L. (Tamar) Weiss, and Oren Zuckerman. 2014. ExciteTray: Developing an assistive technology to promote selffeeding among young children. In Proceedings of the 2014 conference on interaction design and children (IDC '14), Association for Computing Machinery, New York, NY, USA, 297-300. DOI:https://doi.org/10.1145/2593968.2610476

*[53] Sangita Ganesh, Paul Marshall, Yvonne Rogers, and Kenton O’Hara. 2014. FoodWorks: Tackling fussy eating by digitally augmenting children's meals. In Proceedings of the 8th nordic conference on humancomputer interaction: Fun, fast, foundational (NordiCHI '14), Association for Computing Machinery, New York, NY, USA, 147-156. DOI:https://doi.org/10.1145/2639189.2639225

[54] Tom Gayler. 2020. Inbodied interaction design example: smell. interactions 27, 2 (February 2020), 38-39. DOI:https://doi.org/10.1145/3380870

*[55] Tom Gayler and Corina Sas. 2017. An Exploration of Taste-emotion Mappings from the Perspective of Food Design Practitioners. In Proceedings of the 2Nd ACM SIGCHI International Workshop on Multisensory Approaches to Human-Food Interaction (MHFI 2017), ACM, New York, NY, USA, 23-28. DOI:https://doi.org/10.1145/3141788.3141793

*[56] Tom Gayler, Corina Sas, and Vaiva Kalnikaite. 2019. Taste Your Emotions: An Exploration of the Relationship Between Taste and Emotional Experience for HCI. In Proceedings of the 2019 on Designing Interactive Systems Conference (DIS '19), ACM, New York, NY, USA, 1279-1291. DOI:https://doi.org/10.1145/3322276.3322336

[57] Tom Gayler, Corina Sas, and Vaiva Kalnikaite. 2020. Material Food Probe: Personalized 3D Printed Flavors for Emotional Communication in Intimate Relationships. In Proceedings of the 2020 ACM on Designing 
Interactive Systems Conference (DIS '20), Association for Computing Machinery, Eindhoven, Netherlands, 965-978. DOI:https://doi.org/10.1145/3357236.3395533

[58] Tom Gayler, Corina Sas, and Vaiva Kalnikaite. 2020. Co-Designing Flavor-Based Memory Cues with Older Adults. In Companion Publication of the 2020 International Conference on Multimodal Interaction (ICMI '20 Companion), Association for Computing Machinery, New York, NY, USA, 287-291.

DOI:https://doi.org/10.1145/3395035.3425644

[59] Tom Gayler, Corina Sas, and Vaiva Kalnikaite. 2021. Sensory Probes: An Exploratory Design Research Method for Human-Food Interaction. In Proceedings of the Designing Interactive Systems Conference, ACM New York, NY. DOI:https://doi.org/10.1145/3461778.3462013

[60] Eugene T. Gendlin. 1998. Focusing-oriented psychotherapy: A manual of the experiential method. Guilford Press.

*[61] Doménique van Gennip, Elise van den Hoven, and Panos Markopoulos. 2015. Things that make us reminisce: Everyday memory cues as opportunities for interaction design. In Proceedings of the 33rd annual ACM conference on human factors in computing systems (CHI '15), Association for Computing Machinery, New York, NY, USA, 3443-3452. DOI:https://doi.org/10.1145/2702123.2702460

[62] E. Greimel, M. Macht, E. Krumhuber, and H. Ellgring. 2006. Facial and affective reactions to tastes and their modulation by sadness and joy. Physiology and Behavior 89, 2 (2006), 261-269. DOI:https://doi.org/10.1016/j.physbeh.2006.06.002

[63] Andrea Grimes and Richard Harper. 2008. Celebratory Technology: New Directions for Food Research in HCI. In Proceedings of the SIGCHI Conference on Human Factors in Computing Systems (CHI '08), ACM, New York, NY, USA, 467-476. DOI:https://doi.org/10.1145/1357054.1357130

[64] Shad Gross, Austin Toombs, Jeff Wain, and Kevin Walorski. 2011. Foodmunity: Designing community interactions over food. In CHI '11 extended abstracts on human factors in computing systems (CHI EA '11), Association for Computing Machinery, New York, NY, USA, 1019-1024. DOI:https://doi.org/10.1145/1979742.1979504

[65] Gustav Freytag. 2008. Freytag's Technique of the Drama: An Exposition of Dramatic Composition and Art.

[66] Lilit Hakobyan, Jo Lumsden, Rachel Shaw, and Dympna O’Sullivan. 2016. A longitudinal evaluation of the acceptability and impact of a diet diary app for older adults with age-related macular degeneration. In Proceedings of the 18th international conference on human-computer interaction with mobile devices and services (MobileHCI '16), Association for Computing Machinery, New York, NY, USA, 124-134. DOI:https://doi.org/10.1145/2935334.2935356

*[67] Natsuki Hamanishi, Michinari Kono, Shunichi Suwa, Takashi Miyaki, and Jun Rekimoto. 2018. Flufy: Recyclable and edible rapid prototyping using fluffed sugar. In Proceedings of the 23rd international conference on intelligent user interfaces companion (IUI '18 companion), Association for Computing Machinery, New York, NY, USA. DOI:https://doi.org/10.1145/3180308.3180335

*[68] Seo Yoon Han and Eun Ji Kang. 2017. ChilDish: The Smart Plate and Cup for Children. In Proceedings of the Companion of the 2017 ACM/IEEE International Conference on Human-Robot Interaction (HRI '17), Association for Computing Machinery, New York, NY, USA, 391-392. DOI:https://doi.org/10.1145/3029798.3034946

*[69] Daniel Harley, Alexander Verni, Mackenzie Willis, Ashley Ng, Lucas Bozzo, and Ali Mazalek. 2018. Sensory VR: Smelling, touching, and eating virtual reality. In Proceedings of the twelfth international conference on tangible, embedded, and embodied interaction (TEI '18), Association for Computing Machinery, New York, NY, USA, 386-397. DOI:https://doi.org/10.1145/3173225.3173241

[70] Marc Hassenzahl and Noam Tractinsky. 2006. User experience-a research agenda. Behaviour \& information technology 25, 2 (2006), 91-97.

[71] Samantha J. Heintzelman and Laura A. King. 2013. On knowing more than we can tell: Intuitive processes and the experience of meaning. The Journal of Positive Psychology 8, 6 (2013), 471-482.

[72] Anu Helkkula, Carol Kelleher, and Minna Pihlström. 2012. Practices and experiences: challenges and opportunities for value research. Journal of Service Management (2012).

*[73] Niels Henze, Thomas Olsson, Stefan Schneegass, Alireza Sahami Shirazi, and Kaisa Väänänen-VainioMattila. 2015. Augmenting Food with Information. In Proceedings of the 14th International Conference on Mobile and Ubiquitous Multimedia (MUM '15), ACM, New York, NY, USA, 258-266.

DOI:https://doi.org/10.1145/2836041.2836068 
*[74] Hiromi Nakamura and Homei Miyashita. 2011. Augmented Gustation Using Electricity. In Proceedings of the 2Nd Augmented Human International Conference (AH '11), ACM, New York, NY, USA, 34:1-34:2. DOI:https://doi.org/10.1145/1959826.1959860

*[75] Masaharu Hirose, Karin Iwazaki, Kozue Nojiri, Minato Takeda, Yuta Sugiura, and Masahiko Inami. 2015. Gravitamine spice: A system that changes the perception of eating through virtual weight sensation. In Proceedings of the 6th augmented human international conference (AH '15), Association for Computing Machinery, New York, NY, USA, 33-40. DOI:https://doi.org/10.1145/2735711.2735795

[76] Kristina Höök. 2018. Designing with the Body: Somaesthetic Interaction Design. MIT Press.

[77] Kristina Höök, Martin P. Jonsson, Anna Ståhl, and Johanna Mercurio. 2016. Somaesthetic Appreciation Design. In Proceedings of the 2016 CHI Conference on Human Factors in Computing Systems (CHI '16), Association for Computing Machinery, New York, NY, USA, 3131-3142. DOI:https://doi.org/10.1145/2858036.2858583

*[78] Gijs Huisman, Merijn Bruijnes, and Dirk K. J. Heylen. 2016. A moving feast: Effects of color, shape and animation on taste associations and taste perceptions. In Proceedings of the 13th international conference on advances in computer entertainment technology (ACE '16), Association for Computing Machinery, New York, NY, USA. DOI:https://doi.org/10.1145/3001773.3001776

[79] Annika Hupfeld and Tom Rodden. 2012. Laying the table for HCI: Uncovering ecologies of domestic food consumption. In Proceedings of the SIGCHI conference on human factors in computing systems (CHI '12), Association for Computing Machinery, New York, NY, USA, 119-128. DOI:https://doi.org/10.1145/2207676.2207694

*[80] Youjin Hwang, Siyoung Lee, Hyeong Seok Jeon, Jung Han Yoon Park, Ki Won Lee, and Joonhwan Lee. 2018. "Eat What You Want and Be Healthy!": Comfort food effects: Human-food interaction in view of celebratory technology. In Proceedings of the 3rd international workshop on multisensory approaches to human-food interaction (MHFI'18), Association for Computing Machinery, New York, NY, USA. DOI:https://doi.org/10.1145/3279954.3279958

*[81] Jesús Ibáñez. 2015. Play with your food: Enjoyable meals, eatable games. In Proceedings of the 2015 annual symposium on computer-human interaction in play (CHI PLAY '15), Association for Computing Machinery, New York, NY, USA, 553-558. DOI:https://doi.org/10.1145/2793107.2810294

*[82] Javier Jiménez Villarreal and Sara Ljungblad. 2011. Experience centred design for a robotic eating aid. In Proceedings of the 6th international conference on human-robot interaction (HRI '11), Association for Computing Machinery, New York, NY, USA, 155-156. DOI:https://doi.org/10.1145/1957656.1957708

*[83] Yeong Rae Joi, Beom Taek Jeong, Jin Hwang Kim, Joongsin Park, Juhee Cho, Eunju Seong, Byung-Chull Bae, and Jun Dong Cho. 2016. Interactive and connected tableware for promoting children's vegetable-eating and family interaction. In Proceedings of the the 15th international conference on interaction design and children (IDC '16), Association for Computing Machinery, New York, NY, USA, 414-420. DOI:https://doi.org/10.1145/2930674.2930711

*[84] Azusa Kadomura, Cheng-Yuan Li, Yen-Chang Chen, Koji Tsukada, Itiro Siio, and Hao-hua Chu. 2013. Sensing Fork: Eating Behavior Detection Utensil and Mobile Persuasive Game. In CHI '13 Extended Abstracts on Human Factors in Computing Systems (CHI EA '13), ACM, New York, NY, USA, 1551-1556. DOI:https://doi.org/10.1145/2468356.2468634

*[85] Azusa Kadomura, Cheng-Yuan Li, Koji Tsukada, Hao-Hua Chu, and Itiro Siio. 2014. Persuasive technology to improve eating behavior using a sensor-embedded fork. In Proceedings of the 2014 ACM international joint conference on pervasive and ubiquitous computing (UbiComp '14), Association for Computing Machinery, New York, NY, USA, 319-329. DOI:https://doi.org/10.1145/2632048.2632093

*[86] Azusa Kadomura, Koji Tsukada, and Itiro Siio. 2013. EducaTableware: computer-augmented tableware to enhance the eating experiences. In CHI '13 Extended Abstracts on Human Factors in Computing Systems (CHI EA '13), Association for Computing Machinery, New York, NY, USA, 3071-3074. DOI:https://doi.org/10.1145/2468356.2479613

[87] Vaiva Kalnikaité, Jon Bird, and Yvonne Rogers. 2013. Decision-making in the Aisles: Informing, Overwhelming or Nudging Supermarket Shoppers? Personal Ubiquitous Comput. 17, 6 (August 2013), 1247-1259. DOI:https://doi.org/10.1007/s00779-012-0589-z

*[88] Ravi Karkar, Jessica Schroeder, Daniel A. Epstein, Laura R. Pina, Jeffrey Scofield, James Fogarty, Julie A. Kientz, Sean A. Munson, Roger Vilardaga, and Jasmine Zia. 2017. TummyTrials: A feasibility study of using 
self-experimentation to detect individualized food triggers. In Proceedings of the 2017 CHI conference on human factors in computing systems (CHI '17), Association for Computing Machinery, New York, NY, USA, 6850-6863. DOI:https://doi.org/10.1145/3025453.3025480

[89] Hideaki Kashima and Naoyuki Hayashi. 2011. Basic Taste Stimuli Elicit Unique Responses in Facial Skin Blood Flow. PLOS ONE 6, 12 (December 2011), e28236.

DOI:https://doi.org/10.1371/journal.pone.0028236

*[90] Flavius Kehr, Marc Hassenzahl, Matthias Laschke, and Sarah Diefenbach. 2012. A transformational product to improve self-control strength: The chocolate machine. In Proceedings of the SIGCHI conference on human factors in computing systems (CHI '12), Association for Computing Machinery, New York, NY, USA, 689-694. DOI:https://doi.org/10.1145/2207676.2207774

[91] Erika Kerruish. 2019. Arranging sensations: smell and taste in augmented and virtual reality. The Senses and Society 14, 1 (January 2019), 31-45. DOI:https://doi.org/10.1080/17458927.2018.1556952

*[92] Rohit Ashok Khot, Deepti Aggarwal, Ryan Pennings, Larissa Hjorth, and Florian “Floyd” Mueller. 2017. EdiPulse: Investigating a playful approach to self-monitoring through 3D printed chocolate treats. In Proceedings of the 2017 CHI conference on human factors in computing systems (CHI '17), Association for Computing Machinery, New York, NY, USA, 6593-6607. DOI:https://doi.org/10.1145/3025453.3025980

*[93] Rohit Ashok Khot, Eshita Sri Arza, Harshitha Kurra, and Yan Wang. 2019. FoBo: Towards designing a robotic companion for solo dining. In Extended abstracts of the $2019 \mathrm{CHI}$ conference on human factors in computing systems (CHI EA '19), Association for Computing Machinery, New York, NY, USA, 1-6. DOI:https://doi.org/10.1145/3290607.3313069

*[94] Rohit Ashok Khot, Jeewon Lee, Deepti Aggarwal, Larissa Hjorth, and Florian "Floyd" Mueller. 2015. TastyBeats: Designing palatable representations of physical activity. In Proceedings of the 33rd annual ACM conference on human factors in computing systems (CHI '15), Association for Computing Machinery, New York, NY, USA, 2933-2942. DOI:https://doi.org/10.1145/2702123.2702197

*[95] Rohit Ashok Khot, Jeewon Lee, Larissa Hjorth, and Florian "Floyd" Mueller. 2015. TastyBeats: Celebrating heart rate data with a drinkable spectacle. In Proceedings of the ninth international conference on tangible, embedded, and embodied interaction (TEI '15), Association for Computing Machinery, New York, NY, USA, 229-232. DOI:https://doi.org/10.1145/2677199.2680545

*[96] Rohit Ashok Khot, Jeewon Lee, Helmut Munz, Deepti Aggarwal, and Florian Floyd Mueller. 2014. Tastybeats: Making mocktails with heartbeats. In CHI '14 extended abstracts on human factors in computing systems (CHI EA '14), Association for Computing Machinery, New York, NY, USA, 467-470. DOI:https://doi.org/10.1145/2559206.2574830

[97] Rohit Ashok Khot and Florian Mueller. 2019. Human-Food Interaction. Now Publishers, Norwell, MA, UNITED STATES. Retrieved May 11, 2020 from http://ebookcentral.proquest.com/lib/lancaster/detail.action?docID=5892662

*[98] Rohit Ashok Khot, Ryan Pennings, and Florian "Floyd" Mueller. 2015. EdiPulse: Supporting physical activity with chocolate printed messages. In Proceedings of the 33rd annual ACM conference extended abstracts on human factors in computing systems (CHI EA '15), Association for Computing Machinery, New York, NY, USA, 1391-1396. DOI:https://doi.org/10.1145/2702613.2732761

*[99] Rohit Ashok Khot, Ryan Pennings, and Florian "Floyd" Mueller. 2015. EdiPulse: Turning physical activity into chocolates. In Proceedings of the 33rd annual ACM conference extended abstracts on human factors in computing systems (CHI EA '15), Association for Computing Machinery, New York, NY, USA, 331-334. DOI:https://doi.org/10.1145/2702613.2725436

*[100] Jaejeung Kim, Joonyoung Park, and Uichin Lee. 2016. EcoMeal: A Smart Tray for Promoting Healthy Dietary Habits. In Proceedings of the 2016 CHI Conference Extended Abstracts on Human Factors in Computing Systems (CHI EA '16), Association for Computing Machinery, New York, NY, USA, 2165-2170. DOI:https://doi.org/10.1145/2851581.2892310

*[101] Joohee Kim, Kwang-Jae Lee, Mankyung Lee, Nahyeon Lee, Byung-Chull Bae, Genehee Lee, Juhee Cho, Young Mog Shim, and Jun-Dong Cho. 2016. Slowee: A Smart Eating-Speed Guide System with Light and Vibration Feedback. In Proceedings of the 2016 CHI Conference Extended Abstracts on Human Factors in Computing Systems (CHI EA '16), Association for Computing Machinery, New York, NY, USA, 2563-2569. DOI:https://doi.org/10.1145/2851581.2892323 
[102] Barbara Kirshenblatt-Gimblett. 1999. Playing to the Senses: Food as a Performance Medium. Performance Research 4, 1 (January 1999), 1-30. DOI:https://doi.org/10.1080/13528165.1999.10871639

[103] Yui Kita and Jun Rekimoto. 2013. Spot-light: multimodal projection mapping on food. In International Conference on Human-Computer Interaction, Springer, 652-655.

*[104] Naoya Koizumi, Hidekazu Tanaka, Yuji Uema, and Masahiko Inami. 2011. Chewing jockey: Augmented food texture by using sound based on the cross-modal effect. In Proceedings of the 8th international conference on advances in computer entertainment technology (ACE '11), Association for Computing Machinery, New York, NY, USA. DOI:https://doi.org/10.1145/2071423.2071449

*[105] Tom L. Koller, Maria Kyrarini, and Axel Gräser. 2019. Towards robotic drinking assistance: low cost multisensor system to limit forces in human-robot-interaction. In Proceedings of the 12th ACM International Conference on PErvasive Technologies Related to Assistive Environments (PETRA '19), Association for Computing Machinery, New York, NY, USA, 243-246. DOI:https://doi.org/10.1145/3316782.3321539

*[106] Dannie Korsgaard, Thomas Bjørner, Pernille Krog Sørensen, and Jon Ram Bruun-Pedersen. 2019. Older adults eating together in a virtual living room: Opportunities and limitations of eating in augmented virtuality. In Proceedings of the 31st european conference on cognitive ergonomics (ECCE 2019), Association for Computing Machinery, New York, NY, USA, 168-176. DOI:https://doi.org/10.1145/3335082.3335093

[107] Carolyn Korsmeyer. 1999. Making Sense of Taste: Food and Philosophy. Cornell University Press. Retrieved June 21, 2021 from https://www.jstor.org/stable/10.7591/j.ctt5hh0p6

[108] Christian Kray, Keith Cheverst, Dan Fitton, Corina Sas, John Patterson, Mark Rouncefield, and Christoph Stahl. 2006. Sharing control of dispersed situated displays between nand residential users. In Proceedings of the 8th conference on Human-computer interaction with mobile devices and services (MobileHCI '06), Association for Computing Machinery, Helsinki, Finland, 61-68. DOI:https://doi.org/10.1145/1152215.1152229

[109] Stacey Kuznetsov, Christina J. Santana, and Elenore Long. 2016. Everyday food science as a design space for community literacy and habitual sustainable practice. In Proceedings of the 2016 CHI conference on human factors in computing systems (CHI '16), Association for Computing Machinery, New York, NY, USA, 1786-1797. DOI:https://doi.org/10.1145/2858036.2858363

*[110] Win Tun Latt, Trieu Phat Luu, Christopher Kuah, and Ang Wei Tech. 2014. Towards an upper-limb exoskeleton system for assistance in activities of daily living (ADLs). In Proceedings of the international convention on rehabilitation engineering \&amp; assistive technology (i-CREATe 2014), Singapore Therapeutic, Assistive \&amp; Rehabilitative Technologies (START) Centre, Midview City, SGP.

*[111] Yujin Lee, Jee Bin Yim, Daye Kang, HyeonBeom Yi, and Daniel Saakes. 2019. Designing internal structure of chocolate and its effect on food texture. In Companion publication of the 2019 on designing interactive systems conference 2019 companion (DIS '19 companion), Association for Computing Machinery, New York, NY, USA, 231-235. DOI:https://doi.org/10.1145/3301019.3323896

[112] Ying-Ju Lin, Parinya Punpongsanon, Xin Wen, Daisuke Iwai, Kosuke Sato, Marianna Obrist, and Stefanie Mueller. 2020. FoodFab: Creating Food Perception Illusions using Food 3D Printing. In Proceedings of the 2020 CHI Conference on Human Factors in Computing Systems (CHI '20), Association for Computing Machinery, Honolulu, HI, USA, 1-13. DOI:https://doi.org/10.1145/3313831.3376421

[113] Ying-Li Lin, Tsai-Yi Chou, Yu-Cheng Lieo, Yu-Cheng Huang, and Ping-Hsuan Han. 2018. TransFork: Using Olfactory Device for Augmented Tasting Experience with Video See-through Head-mounted Display. In Proceedings of the 24th ACM Symposium on Virtual Reality Software and Technology (VRST '18), ACM, New York, NY, USA, 58:1-58:2. DOI:https://doi.org/10.1145/3281505.3281560

*[114] Jin-Ling Lo, Tung-Yun Lin, Hao-Hua Chu, Hsi-Chin Chou, Jen-Hao Chen, Jane Yung-Jen Hsu, and Polly Huang. 2007. Playful tray: Adopting ubicomp and persuasive techniques into play-based occupational therapy for reducing poor eating behavior in young children. In Proceedings of the 9th international conference on ubiquitous computing (UbiComp '07), Springer-Verlag, Berlin, Heidelberg, 38-55.

[115] Michal Luria, Amit Zoran, and Jodi Forlizzi. 2019. Challenges of Designing HCI for Negative Emotions.

[116] Filippo Marinetti. 2016. The Manifesto of Futurist Cooking. Passerino Editore.

[117] David Marshall. 2005. Food as ritual, routine or convention. Consumption Markets \& Culture 8, 1 (March 2005), 69-85. DOI:https://doi.org/10.1080/10253860500069042 
*[118] Signe Lund Mathiesen, Derek Victor Byrne, and Qian Janice Wang. 2019. Sonic Mug: A Sonic Seasoning System. In Proceedings of the 14th International Audio Mostly Conference: A Journey in Sound (AM'19), Association for Computing Machinery, New York, NY, USA, 264-267. DOI:https://doi.org/10.1145/3356590.3356634

*[119] Yash Dhanpal Mehta, Rohit Ashok Khot, Rakesh Patibanda, and Florian "Floyd" Mueller. 2018. Arm-a-dine: Towards understanding the design of playful embodied eating experiences. In Proceedings of the 2018 annual symposium on computer-human interaction in play (CHI PLAY '18), Association for Computing Machinery, New York, NY, USA, 299-313. DOI:https://doi.org/10.1145/3242671.3242710

[120] Maurice Merleau-Ponty. 1996. Phenomenology of perception. Motilal Banarsidass Publishe.

*[121] Bruno Mesz, Kevin Herzog, Juan Cruz Amusátegui, Lucas Samaruga, and Sebastián Tedesco. 2017. Let’s Drink This Song Together: Interactive Taste-sound Systems. In Proceedings of the 2Nd ACM SIGCHI International Workshop on Multisensory Approaches to Human-Food Interaction (MHFI 2017), ACM, New York, NY, USA, 13-17. DOI:https://doi.org/10.1145/3141788.3141791

*[122] Robb Mitchell, Alexandra Papadimitriou, Youran You, and Laurens Boer. 2015. Really eating together: A kinetic table to synchronise social dining experiences. In Proceedings of the 6th augmented human international conference (AH '15), Association for Computing Machinery, New York, NY, USA, 173-174. DOI:https://doi.org/10.1145/2735711.2735822

*[123] Christiane Moser and Manfred Tscheligi. 2013. Playful Taste Interaction. In Proceedings of the 12th International Conference on Interaction Design and Children (IDC '13), ACM, New York, NY, USA, 340-343. DOI:https://doi.org/10.1145/2485760.2485828

*[124] Florian "Floyd" Mueller, Tuomas Kari, Rohit Khot, Zhuying Li, Yan Wang, Yash Mehta, and Peter Arnold. 2018. Towards experiencing eating as a form of play. In Proceedings of the 2018 annual symposium on computer-human interaction in play companion extended abstracts (CHI PLAY'18 extended abstracts), Association for Computing Machinery, New York, NY, USA, 559-567. DOI:https://doi.org/10.1145/3270316.3271528

*[125] Martin Murer, Ilhan Aslan, and Manfred Tscheligi. 2013. LOLLio: Exploring Taste As Playful Modality. In Proceedings of the 7th International Conference on Tangible, Embedded and Embodied Interaction (TEI '13), ACM, New York, NY, USA, 299-302. DOI:https://doi.org/10.1145/2460625.2460675

*[126] Hiromi Nakamura and Homei Miyashita. 2011. Communication by Change in Taste. In CHI '11 Extended Abstracts on Human Factors in Computing Systems (CHI EA '11), ACM, New York, NY, USA, 1999-2004. DOI:https://doi.org/10.1145/1979742.1979916

*[127] Hiromi Nakamura and Homei Miyashita. 2012. Development and evaluation of interactive system for synchronizing electric taste and visual content. In Proceedings of the SIGCHI conference on human factors in computing systems (CHI '12), Association for Computing Machinery, New York, NY, USA, 517-520. DOI:https://doi.org/10.1145/2207676.2207747

*[128] Hiromi Nakamura and Homei Miyashita. 2013. Enhancing saltiness with cathodal current. In CHI'13 extended abstracts on human factors in computing systems (CHI EA '13), Association for Computing Machinery, New York, NY, USA, 3111-3114. DOI:https://doi.org/10.1145/2468356.2479623

[129] Kizashi Nakano, Daichi Horita, Nobuchika Sakata, Kiyoshi Kiyokawa, Keiji Yanai, and Takuji Narumi. 2019. DeepTaste: Augmented Reality Gustatory Manipulation with GAN-Based Real-Time Food-to-Food Translation. In 2019 IEEE International Symposium on Mixed and Augmented Reality (ISMAR), 212-223. DOI:https://doi.org/10.1109/ISMAR.2019.000-1

*[130] Takuji Narumi. 2016. Multi-sensorial virtual reality and augmented human food interaction. In Proceedings of the 1st workshop on multi-sensorial approaches to human-food interaction (MHFI '16), Association for Computing Machinery, New York, NY, USA. DOI:https://doi.org/10.1145/3007577.3007587

*[131] Takuji Narumi, Yuki Ban, Takashi Kajinami, Tomohiro Tanikawa, and Michitaka Hirose. 2012. Augmented perception of satiety: Controlling food consumption by changing apparent size of food with augmented reality. In Proceedings of the SIGCHI conference on human factors in computing systems (CHI '12), Association for Computing Machinery, New York, NY, USA, 109-118. DOI:https://doi.org/10.1145/2207676.2207693

*[132] Takuji Narumi, Shinya Nishizaka, Takashi Kajinami, Tomohiro Tanikawa, and Michitaka Hirose. 2011. Augmented Reality Flavors: Gustatory Display Based on Edible Marker and Cross-modal Interaction. In 
Proceedings of the SIGCHI Conference on Human Factors in Computing Systems (CHI '11), ACM, New York, NY, USA, 93-102. DOI:https://doi.org/10.1145/1978942.1978957

[133] Takuji Narumi, Shinya Nishizaka, Takashi Kajinami, Tomohiro Tanikawa, and Michitaka Hirose. 2011. Meta Cookie+: An Illusion-Based Gustatory Display. In Virtual and Mixed Reality - New Trends (Lecture Notes in Computer Science), Springer, Berlin, Heidelberg, 260-269. DOI:https://doi.org/10.1007/978-3642-22021-0_29

[134] Takuji Narumi, Shinya Nishizaka, Takashi Kajinami, Tomohiro Tanikawa, and Michitaka Hirose. 2011. MetaCookie+. In 2011 IEEE Virtual Reality Conference, 265-266. DOI:https://doi.org/10.1109/VR.2011.5759500

*[135] Takuji Narumi, Munehiko Sato, Tomohiro Tanikawa, and Michitaka Hirose. 2010. Evaluating crosssensory perception of superimposing virtual color onto real drink: Toward realization of pseudogustatory displays. In Proceedings of the 1st augmented human international conference (AH '10), Association for Computing Machinery, New York, NY, USA. DOI:https://doi.org/10.1145/1785455.1785473

*[136] Mamoun Nawahdah and Tomoo Inoue. 2013. Virtually dining together in time-shifted environment: KIZUNA design. In Proceedings of the 2013 conference on computer supported cooperative work (CSCW '13), Association for Computing Machinery, New York, NY, USA, 779-788. DOI:https://doi.org/10.1145/2441776.2441863

*[137] Masahiro Nishizawa, Wanting Jiang, and Katsunori Okajima. 2016. Projective-ar system for customizing the appearance and taste of food. In Proceedings of the 2016 workshop on multimodal virtual and augmented reality (MVAR '16), Association for Computing Machinery, New York, NY, USA. DOI:https://doi.org/10.1145/3001959.3001966

[138] Jon Noronha, Eric Hysen, Haoqi Zhang, and Krzysztof Z. Gajos. 2011. Platemate: Crowdsourcing nutritional analysis from food photographs. In Proceedings of the 24th annual ACM symposium on user interface software and technology (UIST '11), Association for Computing Machinery, New York, NY, USA, 1-12. DOI:https://doi.org/10.1145/2047196.2047198

*[139] Marianna Obrist, Rob Comber, Sriram Subramanian, Betina Piqueras-Fiszman, Carlos Velasco, and Charles Spence. 2014. Temporal, affective, and embodied characteristics of taste experiences: A framework for design. In Proceedings of the SIGCHI conference on human factors in computing systems (CHI '14), Association for Computing Machinery, New York, NY, USA, 2853-2862. DOI:https://doi.org/10.1145/2556288.2557007

[140] Marianna Obrist, Carlos Velasco, Chi Thanh Vi, Nimesha Ranasinghe, Ali Israr, Adrian D. Cheok, Charles Spence, and Ponnampalam Gopalakrishnakone. 2016. Touch, Taste, \& Smell User Interfaces: The Future of Multisensory HCI. In Proceedings of the 2016 CHI Conference Extended Abstracts on Human Factors in Computing Systems (CHI EA '16), ACM, New York, NY, USA, 3285-3292. DOI:https://doi.org/10.1145/2851581.2856462

*[141] Naoshi Ooba, Kazuma Aoyama, Hiromi Nakamura, and Homei Miyashita. 2018. Unlimited Electric Gum: A Piezo-based Electric Taste Apparatus Activated by Chewing. In The 31st Annual ACM Symposium on User Interface Software and Technology Adjunct Proceedings (UIST '18 Adjunct), Association for Computing Machinery, New York, NY, USA, 157-159. DOI:https://doi.org/10.1145/3266037.3271635

*[142] Gaurav Patekar and Karan Dudeja. 2017. Data jalebi bot. In SIGGRAPH asia 2017 posters (SA '17), Association for Computing Machinery, New York, NY, USA. DOI:https://doi.org/10.1145/3145690.3145737

*[143] Gaurav Patekar, Karan Dudeja, Himanshu Bablani, and Debanshu Bhaumik. 2018. Data Jalebi Bot. In Extended Abstracts of the 2018 CHI Conference on Human Factors in Computing Systems (CHI EA '18), Association for Computing Machinery, New York, NY, USA, 1-4. DOI:https://doi.org/10.1145/3170427.3186528

*[144] Nur Ellyza Abd Rahman, Azhri Azhar, Kasun Karunanayaka, Adrian David Cheok, Mohammad Abdullah Mohamad Johar, Jade Gross, and Andoni Luis Aduriz. 2016. Implementing new food interactions using magnetic dining table platform and magnetic foods. In Proceedings of the 2016 workshop on Multimodal Virtual and Augmented Reality (MVAR '16), Association for Computing Machinery, New York, NY, USA, 13. DOI:https://doi.org/10.1145/3001959.3001965 
[145] Jörgen Rahm-Skågeby and Lina Rahm. 2021. HCI and deep time: toward deep time design thinking. Human-Computer Interaction 0, 0 (April 2021), 1-14. DOI:https://doi.org/10.1080/07370024.2021.1902328

*[146] Nimesha Ranasinghe, Adrian David Cheok, Owen Noel Newton Fernando, Hideaki Nii, and Gopalakrishnakone Ponnampalam. 2011. Electronic taste stimulation. In Proceedings of the 13th international conference on Ubiquitous computing (UbiComp '11), Association for Computing Machinery, New York, NY, USA, 561-562. DOI:https://doi.org/10.1145/2030112.2030213

*[147] Nimesha Ranasinghe, Adrian David Cheok, and Ryohei Nakatsu. 2012. Taste/IP: The sensation of taste for digital communication. In Proceedings of the 14th ACM international conference on multimodal interaction (ICMI '12), Association for Computing Machinery, New York, NY, USA, 409-416. DOI:https://doi.org/10.1145/2388676.2388768

*[148] Nimesha Ranasinghe, Adrian Cheok, Ryohei Nakatsu, and Ellen Yi-Luen Do. 2013. Simulating the sensation of taste for immersive experiences. In Proceedings of the 2013 ACM international workshop on immersive media experiences (ImmersiveMe '13), Association for Computing Machinery, New York, NY, USA, 29-34. DOI:https://doi.org/10.1145/2512142.2512148

*[149] Nimesha Ranasinghe and Ellen Yi-Luen Do. 2017. Digital Lollipop: Studying Electrical Stimulation on the Human Tongue to Simulate Taste Sensations. ACM Trans. Multimedia Comput. Commun. Appl. 13, 1 (January 2017), 1-22. DOI:https://doi.org/10.1145/2996462

*[150] Nimesha Ranasinghe, Pravar Jain, Shienny Karwita, and Ellen Yi-Luen Do. 2017. Virtual lemonade: Let's teleport your lemonade! In Proceedings of the eleventh international conference on tangible, embedded, and embodied interaction (TEI '17), Association for Computing Machinery, New York, NY, USA, 183-190. DOI:https://doi.org/10.1145/3024969.3024977

*[151] Nimesha Ranasinghe, Kasun Karunanayaka, Adrian David Cheok, Owen Noel Newton Fernando, Hideaki Nii, and Ponnampalam Gopalakrishnakone. 2011. Digital taste and smell communication. In Proceedings of the 6th international conference on body area networks (BodyNets '11), ICST (Institute for Computer Sciences, Social-Informatics and Telecommunications Engineering), Brussels, BEL, 78-84.

*[152] Nimesha Ranasinghe, Kasun Karunanayaka, Adrian David Cheok, Owen Noel Newton Fernando, Hideaki Nii, and Gopalakrishnakone Ponnampalam. 2011. Digital taste \& smell for remote multisensory interactions: Poster abstract. In Proceedings of the 6th international conference on body area networks (BodyNets '11), ICST (Institute for Computer Sciences, Social-Informatics and Telecommunications Engineering), Brussels, BEL, 128-129.

*[153] Nimesha Ranasinghe, Kuan-Yi Lee, and Ellen Yi-Luen Do. 2014. FunRasa: an interactive drinking platform. In Proceedings of the 8th International Conference on Tangible, Embedded and Embodied Interaction (TEI '14), Association for Computing Machinery, New York, NY, USA, 133-136. DOI:https://doi.org/10.1145/2540930.2540939

*[154] Nimesha Ranasinghe, Kuan-Yi Lee, Gajan Suthokumar, and Ellen Yi-Luen Do. 2014. Taste+: Digitally enhancing taste sensations of food and beverages. In Proceedings of the 22nd ACM international conference on multimedia (MM '14), Association for Computing Machinery, New York, NY, USA, 737-738. DOI:https://doi.org/10.1145/2647868.2654878

*[155] Nimesha Ranasinghe, Kuan-Yi Lee, Gajan Suthokumar, and Ellen Yi-Luen Do. 2014. The Sensation of Taste in the Future of Immersive Media. In Proceedings of the 2nd ACM International Workshop on Immersive Media Experiences (ImmersiveMe '14), Association for Computing Machinery, New York, NY, USA, 7-12. DOI:https://doi.org/10.1145/2660579.2660586

*[156] Nimesha Ranasinghe, Thi Ngoc Tram Nguyen, Yan Liangkun, Lien-Ya Lin, David Tolley, and Ellen Yi-Luen Do. 2017. Vocktail: A virtual cocktail for pairing digital taste, smell, and color sensations. In Proceedings of the 25th ACM international conference on multimedia (MM '17), Association for Computing Machinery, New York, NY, USA, 1139-1147. DOI:https://doi.org/10.1145/3123266.3123440

*[157] Nimesha Ranasinghe, Gajan Suthokumar, Kuan-Yi Lee, and Ellen Yi-Luen Do. 2015. Digital Flavor: Towards Digitally Simulating Virtual Flavors. In Proceedings of the 2015 ACM on International Conference on Multimodal Interaction (ICMI '15), Association for Computing Machinery, New York, NY, USA, 139-146. DOI:https://doi.org/10.1145/2818346.2820761

*[158] Natasha Randall, Swapna Joshi, and Xiaohang Liu. 2018. Health-e-eater: Dinnertime companion robot and magic plate for improving eating habits in children from low-income families. In Companion of the 2018 
ACM/IEEE international conference on human-robot interaction (HRI '18), Association for Computing Machinery, New York, NY, USA, 361-362. DOI:https://doi.org/10.1145/3173386.3177828

*[159] Janet C. Read and Gavin Sim. 2014. Taking the biscuit: Playful interaction. In Proceedings of the 28th international BCS human computer interaction conference on HCI 2014 - sand, sea and sky - holiday HCI (BCS-HCI '14), BCS, Swindon, GBR, 371-375. DOI:https://doi.org/10.14236/ewic/hci2014.36

[160] Andreas Reckwitz. 2002. Toward a theory of social practices: A development in culturalist theorizing. European journal of social theory 5, 2 (2002), 243-263.

[161] O Robin, S Rousmans, A Dittmar, and E Vernet-Maury. 2003. Gender influence on emotional responses to primary tastes. Physiology \& Behavior 78, 3 (March 2003), 385-393. DOI:https://doi.org/10.1016/S00319384(02)00981-2

[162] Valentina Rognoli. 2010. A Broad Survey on Expressive-sensorial Characterization of Materials for Design Education. METU Journal of Faculty of Architecture 27, 2 (December 2010), 287-300. DOI:https://doi.org/10.4305/METU.JFA.2010.2.16

[163] James A. Russell. 2003. Core affect and the psychological construction of emotion. Psychological Review 110, 1 (January 2003), 145-172. DOI:https://doi.org/10.1037/0033-295X.110.1.145

*[164] Annina Rüst. 2014. A piece of the pie chart: Feminist robotics. In ACM SIGGRAPH 2014 art gallery (SIGGRAPH '14), Association for Computing Machinery, New York, NY, USA, 360-366. DOI:https://doi.org/10.1145/2601080.2677713

*[165] Sho Sakurai, Takuji Narumi, Yuki Ban, Tomohiro Tanikawa, and Michitaka Hirose. 2015. CalibraTable: Tabletop system for influencing eating behavior. In SIGGRAPH asia 2015 emerging technologies (SA '15), Association for Computing Machinery, New York, NY, USA. DOI:https://doi.org/10.1145/2818466.2818483

*[166] Nur Amira Samshir, Nurafiqah Johari, Kasun Karunanayaka, and Adrian David Cheok. 2016. Thermal sweet taste machine for multisensory internet. In Proceedings of the fourth international conference on human agent interaction (HAI '16), Association for Computing Machinery, New York, NY, USA, 325-328. DOI:https://doi.org/10.1145/2974804.2980503

[167] Pedro Sanches, Axel Janson, Pavel Karpashevich, Camille Nadal, Chengcheng Qu, Claudia Daudén Roquet, Muhammad Umair, Charles Windlin, Gavin Doherty, Kristina Höök, and Corina Sas. 2019. HCI and Affective Health: Taking stock of a decade of studies and charting future research directions. In Proceedings of the 2019 CHI Conference on Human Factors in Computing Systems, ACM, Glasgow Scotland Uk, 1-17. DOI:https://doi.org/10.1145/3290605.3300475

[168] Corina Sas and Alan Dix. 2008. Designing and evaluating mobile phone-based interaction with public displays. In CHI '08 Extended Abstracts on Human Factors in Computing Systems (CHI EA '08), Association for Computing Machinery, Florence, Italy, 3941-3944. DOI:https://doi.org/10.1145/1358628.1358962

[169] Corina Sas, Steve Whittaker, Steven Dow, Jodi Forlizzi, and John Zimmerman. 2014. Generating Implications for Design Through Design Research. In Proceedings of the 32Nd Annual ACM Conference on Human Factors in Computing Systems (CHI '14), ACM, New York, NY, USA, 1971-1980. DOI:https://doi.org/10.1145/2556288.2557357

[170] Debra Satterfield, Sunghyun Kang, Roger Baer, and Nora Ladjahasan. 2008. Food as experience a design and evaluation methodology. In Undisciplined!, Sheffield Hallam University, Sheffield, UK, 202.

[171] Charles Spence. 2010. The multisensory perception of flavour. Psychologist 23, 9 (September 2010), 720723.

[172] Charles Spence, Carmel A. Levitan, Maya U. Shankar, and Massimiliano Zampini. 2010. Does Food Color Influence Taste and Flavor Perception in Humans? Chem. Percept. 3, 1 (March 2010), 68-84. DOI:https://doi.org/10.1007/s12078-010-9067-z

[173] Charles Spence and Betina Piqueras-Fiszman. 2013. Technology at the dining table. (2013), 13.

*[174] Chie Suzuki, Takuji Narumi, Tomohiro Tanikawa, and Michitaka Hirose. 2014. Affecting tumbler: Affecting our flavor perception with thermal feedback. In Proceedings of the 11th conference on advances in computer entertainment technology (ACE '14), Association for Computing Machinery, New York, NY, USA. DOI:https://doi.org/10.1145/2663806.2663825

*[175] Eiji Suzuki, Takuji Narumi, Sho Sakurai, Tomohiro Tanikawa, and Michitaka Hirose. 2014. Illusion cup: Interactive controlling of beverage consumption based on an illusion of volume perception. In Proceedings 
of the 5th augmented human international conference (AH '14), Association for Computing Machinery, New York, NY, USA. DOI:https://doi.org/10.1145/2582051.2582092

*[176] Nikita Mae B. Tuanquin. 2017. Immersive virtual eating and conditioned food responses. In Proceedings of the 19th ACM international conference on multimodal interaction (ICMI '17), Association for Computing Machinery, New York, NY, USA, 618-622. DOI:https://doi.org/10.1145/3136755.3137029

[177] Junya Ueda and Katsunori Okajima. 2019. AR food changer using deep learning and cross-modal effects. In 2019 IEEE International Conference on Artificial Intelligence and Virtual Reality (AIVR), IEEE, 110-1107.

*[178] Carlos Velasco, Felipe Reinoso Carvalho, Olivia Petit, and Anton Nijholt. 2016. A Multisensory Approach for the Design of Food and Drink Enhancing Sonic Systems. In Proceedings of the 1st Workshop on Multisensorial Approaches to Human-Food Interaction (MHFI '16), ACM, New York, NY, USA, 7:1-7:7. DOI:https://doi.org/10.1145/3007577.3007578

[179] Carlos Velasco, Charles Michel, Jozef Youssef, Xavier Gamez, Adrian David Cheok, and Charles Spence. 2016. Colour-taste correspondences: Designing food experiences to meet expectations or to surprise. International Journal of Food Design 1, 2 (2016), 83-103.

*[180] Carlos Velasco, Yunwen Tu, and Marianna Obrist. 2018. Towards multisensory storytelling with taste and flavor. In Proceedings of the 3rd international workshop on multisensory approaches to human-food interaction (MHFI'18), Association for Computing Machinery, New York, NY, USA. DOI:https://doi.org/10.1145/3279954.3279956

*[181] Chi Thanh Vi, Damien Ablart, Daniel Arthur, and Marianna Obrist. 2017. Gustatory interface: The challenges of 'How' to stimulate the sense of taste. In Proceedings of the 2nd ACM SIGCHI international workshop on multisensory approaches to human-food interaction (MHFI 2017), Association for Computing Machinery, New York, NY, USA, 29-33. DOI:https://doi.org/10.1145/3141788.3141794

*[182] Chi Thanh Vi, Daniel Arthur, and Marianna Obrist. 2018. TasteBud: Bring taste back into the game. In Proceedings of the 3rd international workshop on multisensory approaches to human-food interaction (MHFI'18), Association for Computing Machinery, New York, NY, USA. DOI:https://doi.org/10.1145/3279954.3279955

*[183] Chi Thanh Vi, Asier Marzo, Damien Ablart, Gianluca Memoli, Sriram Subramanian, Bruce Drinkwater, and Marianna Obrist. 2017. TastyFloats: A contactless food delivery system. In Proceedings of the 2017 ACM international conference on interactive surfaces and spaces (ISS '17), Association for Computing Machinery, New York, NY, USA, 161-170. DOI:https://doi.org/10.1145/3132272.3134123

[184] Chi Thanh Vi, Asier Marzo, Gianluca Memoli, Emanuela Maggioni, Damien Ablart, Martin Yeomans, and Marianna Obrist. 2020. LeviSense: A platform for the multisensory integration in levitating food and insights into its effect on flavour perception. International Journal of Human-Computer Studies 139, (2020), 102428.

*[185] Qian Janice Wang, Bruno Mesz, and Charles Spence. 2017. Assessing the Impact of Music on Basic Taste Perception Using Time Intensity Analysis. In Proceedings of the 2Nd ACM SIGCHI International Workshop on Multisensory Approaches to Human-Food Interaction (MHFI 2017), ACM, New York, NY, USA, 18-22. DOI:https://doi.org/10.1145/3141788.3141792

[186] Qian Janice Wang, Sheila Wang, and Charles Spence. 2016. "Turn Up the Taste”: Assessing the Role of Taste Intensity and Emotion in Mediating Crossmodal Correspondences between Basic Tastes and Pitch. Chemical Senses 41, 4 (May 2016), 345-356. DOI:https://doi.org/10.1093/chemse/bjw007

*[187] Yan Wang, Zhuying Li, Robert Jarvis, Rohit Ashok Khot, and Florian “Floyd" Mueller. 2018. The Singing Carrot: Designing Playful Experiences with Food Sounds. In Proceedings of the 2018 Annual Symposium on Computer-Human Interaction in Play Companion Extended Abstracts (CHI PLAY '18 Extended Abstracts), Association for Computing Machinery, New York, NY, USA, 669-676. DOI:https://doi.org/10.1145/3270316.3271512

*[188] Yan Wang, Zhuying Li, Robert Jarvis, Rohit Ashok Khot, and Florian "Floyd" Mueller. 2019. IScream!: Towards the design of playful gustosonic experiences with ice cream. In Extended abstracts of the 2019 CHI conference on human factors in computing systems (CHI EA '19), Association for Computing Machinery, New York, NY, USA, 1-4. DOI:https://doi.org/10.1145/3290607.3313244

[189] Yan Wang, Zhuying Li, Robert S. Jarvis, Joseph La Delfa, Rohit Ashok Khot, and Florian Floyd Mueller. 2020. WeScream! Toward Understanding the Design of Playful Social Gustosonic Experiences with Ice Cream. In Proceedings of the 2020 ACM Designing Interactive Systems Conference (DIS '20), Association for 
Computing Machinery, Eindhoven, Netherlands, 951-963.

DOI:https://doi.org/10.1145/3357236.3395456

[190] Yan Wang, Xian Zhang, Zhuying Li, Rohit Ashok Khot, and Florian "Floyd" Mueller. 2020. Towards a Framework for Designing Playful Gustosonic Experiences. In Extended Abstracts of the $2020 \mathrm{CHI}$ Conference on Human Factors in Computing Systems (CHI EA '20), Association for Computing Machinery, New York, NY, USA, 1-9. DOI:https://doi.org/10.1145/3334480.3382819

*[191] Yong-Xiang Wang, Li-Yun Lo, and Min-Chun Hu. 2014. Eat as much as you can: A kinect-based facial rehabilitation game based on mouth and tongue movements. In Proceedings of the 22nd ACM international conference on multimedia (MM '14), Association for Computing Machinery, New York, NY, USA, 743-744. DOI:https://doi.org/10.1145/2647868.2654887

*[192] Yun Wang, Xiaojuan Ma, Qiong Luo, and Huamin Qu. 2016. Data Edibilization: Representing Data with Food. In Proceedings of the 2016 CHI Conference Extended Abstracts on Human Factors in Computing Systems (CHI EA '16), ACM, New York, USA, 409-422. DOI:https://doi.org/10.1145/2851581.2892570

[193] Alan Warde. 2013. What sort of a practice is eating. Sustainable practices: Social theory and climate change 95, (2013), 17.

*[194] Jun Wei, Adrian David Cheok, and Ryohei Nakatsu. 2012. Let's have dinner together: Evaluate the mediated co-dining experience. In Proceedings of the 14th ACM international conference on multimodal interaction (ICMI '12), Association for Computing Machinery, New York, NY, USA, 225-228. DOI:https://doi.org/10.1145/2388676.2388721

*[195] Jun Wei, Xiaojuan Ma, and Shengdong Zhao. 2014. Food messaging: Using edible medium for social messaging. In Proceedings of the SIGCHI conference on human factors in computing systems (CHI '14), Association for Computing Machinery, New York, NY, USA, 2873-2882. DOI:https://doi.org/10.1145/2556288.2557026

*[196] Jun Wei, Roshan Lalintha Peiris, Jeffrey Tzu Kwan Valino Koh, Xuan Wang, Yongsoon Choi, Xavier Roman Martinez, Remi Tache, Veronica Halupka, and Adrian David Cheok. 2011. Food media: Exploring interactive entertainment over telepresent dinner. In Proceedings of the 8th international conference on advances in computer entertainment technology (ACE '11), Association for Computing Machinery, New York, NY, USA. DOI:https://doi.org/10.1145/2071423.2071455

*[197] Jun Wei, Xuan Wang, Roshan Lalintha Peiris, Yongsoon Choi, Xavier Roman Martinez, Remi Tache, Jeffrey Tzu Kwan Valino Koh, Veronica Halupka, and Adrian David Cheok. 2011. CoDine: An interactive multisensory system for remote dining. In Proceedings of the 13th international conference on ubiquitous computing (UbiComp '11), Association for Computing Machinery, New York, NY, USA, 21-30. DOI:https://doi.org/10.1145/2030112.2030116

[198] Mikael Wiberg, Hiroshi Ishii, Paul Dourish, Anna Vallgårda, Tobie Kerridge, Petra Sundström, Daniela Rosner, and Mark Rolston. 2013. Materiality matters---experience materials. interactions 20, 2 (March 2013), 54-57. DOI:https://doi.org/10.1145/2427076.2427087

[199] Danielle Wilde and Ferran Altarriba Bertran. 2019. Participatory Research through Gastronomy Design: A designerly move towards more playful gastronomy. International Journal of Food Design 4, 1 (2019), 3-37.

[200] Graham Wilson and Stephen A. Brewster. 2017. Multi-moji: Combining Thermal, Vibrotactile \& Visual Stimuli to Expand the Affective Range of Feedback. ACM Press, 1743-1755. DOI:https://doi.org/10.1145/3025453.3025614

[201] Martin R. Yeomans, Lucy Chambers, Heston Blumenthal, and Anthony Blake. 2008. The role of expectancy in sensory and hedonic evaluation: The case of smoked salmon ice-cream. Food Quality and Preference 19, 6 (September 2008), 565-573. D0I:https://doi.org/10.1016/j.foodqual.2008.02.009

*[202] Chawchen Zhaochen, Ding Ting. 2017. Alternative 3D education for children: Course design of 3D printing interactivity for beijing's primary schools. In Proceedings of the fifth international symposium of chinese CHI (Chinese CHI 2017), Association for Computing Machinery, New York, NY, USA, 30-35. DOI:https://doi.org/10.1145/3080631.3080637

*[203] Amit Zoran and Dror Cohen. 2018. Digital konditorei: Programmable taste structures using a modular mold. In Proceedings of the 2018 CHI conference on human factors in computing systems (CHI '18), Association for Computing Machinery, New York, NY, USA, 1-9. DOI:https://doi.org/10.1145/3173574.3173974

[204] Miracle Berry Tablets UK. Retrieved September 14, 2017 from http://mymberry.co.uk/ 